\title{
Representações da álgebra de Lie de campos vetoriais sobre um toro $\mathrm{N}$-dimensional
}

\author{
André Eduardo Zaidan
}

\author{
DISSERTAÇÃO APRESENTADA \\ $\mathrm{AO}$ \\ InstituTO DE MATEMÁTICA E ESTATÍsTICA \\ DA \\ Universidade DE SÃo PAUlo \\ PARA \\ OBTENÇÃO DO TÍTULO \\ DE \\ MESTRE EM CIÊNCIAS
}

Programa: Matemática

Orientador: Prof. Dr. Vyacheslav Futorny

Durante o desenvolvimento deste trabalho

o autor recebeu auxílio financeiro do CNPQ, processo 134228/2013-8.

São Paulo, março de 2015 


\section{Representações da álgebra de Lie de campos vetoriais sobre um toro $\mathrm{N}$-dimensional}

Esta versão da tese contém as correções e alterações sugeridas pela Comissão Julgadora durante a defesa da versão original do trabalho,
realizada em $30 / 03 / 2015$. Uma cópia da versão original está disponível no
Instituto de Matemática e Estatística da Universidade de São Paulo.

Comissão Julgadora:

- Prof. Dr. Vyacheslav Futorny (orientador) - IME-USP

- Prof. Dr. Plamen Koshlukov - UNICAMP

- Prof. Dr. Renato Alessandro Martins - UNIFESP 


\section{Resumo}

Zaidan, A. E. Representações da álgebra de Lie de campos vetoriais sobre um toro N-dimensional. 2015. 39 f. Dissertação (Mestrado) - Instituto de Matemática e Estatística, Universidade de São Paulo, São Paulo, 2015.

O objetivo deste texto é apresentar uma classe de módulos para álgebra de Lie de campos vetoriais em um toro $N$-dimensional, $\operatorname{Vect}\left(\mathbb{T}^{N}\right)$. O caso $N=1$ nos dá a famosa álgebra de Witt (sua extensão central é álgebra de Virasoro). A álgebra Vect $\left(\mathbb{T}^{N}\right)$ apresenta uma classe de módulos parametrizada por módulos de dimensão finita da álgebra $\mathfrak{g l}_{N}$.

Nosso objeto central de estudo são módulos induzidos dos módulos tensoriais $\operatorname{de} \operatorname{Vect}\left(\mathbb{T}^{N}\right)$ para $\operatorname{Vect}\left(\mathbb{T}^{N+1}\right)$. Estes módulos apresentam um quociente irredutível com espaços de peso de dimensão finita.

A álgebra $\operatorname{Vect}\left(\mathbb{T}^{N}\right)$ apresenta como subálgebra $\widetilde{\mathfrak{s l}}_{N+1}$. Com a restrição da ação $\operatorname{de~} \operatorname{Vect}\left(\mathbb{T}^{N}\right)$ a esta subálgebra obtemos o carácter deste quociente.

Para obter um critério de irredutibilidade e construir sua realização de campo livre, consideramos uma classe de módulos para $\Omega^{1}\left(\mathbb{T}^{N+1}\right) / \mathrm{d} \Omega^{0}\left(\mathbb{T}^{N+1}\right) \rtimes \operatorname{Vect}\left(\mathbb{T}^{N}\right)$, construída a partir de álgebras de vértice. Quando restritos a $\operatorname{Vect}\left(\mathbb{T}^{N}\right)$ estes módulos continuam irredutíveis a menos que apareçam no chiral de de Rham.

Palavras-chave: Álgebra Toroidal Completa, Âlgebra de Vértice, Álgebra de Witt, Chiral de de Rham, Módulos Tensoriais. 


\section{Abstract}

Zaidan, A. E.Representation of the Lie Algebra of Vector Fields on a $N$-Dimensional Torus. 2015. 39 f. Dissertação (Mestrado) - Instituto de Matemática e Estatística, Universidade de São Paulo, São Paulo, 2015.

The goal of this text is to present a class of modules for the Lie algebra of vector fields in a $N$-dimensional torus, Vect $\left(\mathbb{T}^{N}\right)$. The case $N=1$ give us the famous Witt algebra (its central extension is the Virasoro algebra). The algebra $\operatorname{Vect}\left(\mathbb{T}^{N}\right)$ has a class of modules parametrized by finite dimensional $\mathfrak{g l}_{N}$-modules.

The central object of our study are modules induced from tensor modules for $\operatorname{Vect}\left(\mathbb{T}^{N}\right)$ to $\operatorname{Vect}\left(\mathbb{T}^{N+1}\right)$. Those modules have an irreducible quotient such that every weight space has finite dimension.

The algebra $\operatorname{Vect}\left(\mathbb{T}^{N}\right)$ has as subalgebra $\widetilde{\mathfrak{s l}}_{N+1}$. Restricting the action of $\operatorname{Vect}\left(\mathbb{T}^{N}\right)$ to this subalgebra we have the character of this quotient.

To obtain a critreria for irreducibility and construct a free field reazilation, we consider a class of modules for $\Omega^{1}\left(\mathbb{T}^{N+1}\right) / \mathrm{d} \Omega^{0}\left(\mathbb{T}^{N+1}\right) \rtimes \operatorname{Vect}\left(\mathbb{T}^{N}\right)$, constructed from vertex algebras. When restricted to $\operatorname{Vect}\left(\mathbb{T}^{N}\right)$ thesse modules remain irreducible, unless they belongs to the chiral de Rham complex. Keywords: Chiral de Rham Complex, Full Toroidal Lie Algebra, Tensor Modules, Vertex Algebra, Witt Algebra. 


\section{Sumário}

Lista de Símbolos vii

Introdução

1 Módulos de Peso e Carácter 3

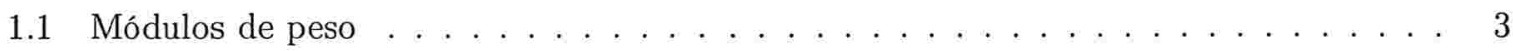

1.2 Módulos de Verma . . . . . . . . . . . . . . . . . . . . . . . 4

1.3 Carácter . . . . . . . . . . . . . . . . . . . . . . 4

2 Álgebra $\operatorname{Vect}\left(\mathbb{T}^{N}\right) \quad 7$

2.1 Álgebra de Witt . . . . . . . . . . . . . . . . . 7

2.2 Extensões Centrais e Generalização da Álgebra de Witt $\ldots \ldots \ldots \ldots$. . . . . 8

2.3 Álgebra Toroidal . . . . . . . . . . . . . . . . . . . . . . . . . 8

3 Módulo Tensorial $\quad 11$

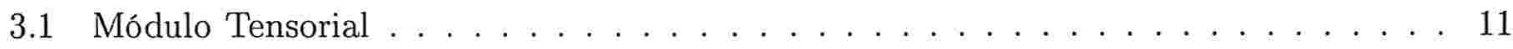

3.2 Complexo de de Rham . . . . . . . . . . . . . . . . . . . 12

$3.3 \mathrm{Um}$ Teorema de Rao . . . . . . . . . . . . . . . . . . . . . . . . 12

3.4 Carácteres dos Submódulos . . . . . . . . . . . . . . . . . . . . . 14

4 Superálgebra de Vértice $\quad 15$

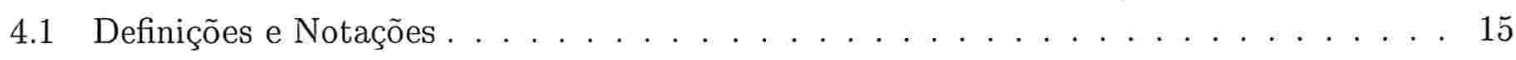

4.2 Superálgebras de Lie de Vértice . . . . . . . . . . . . . . . . . . 16

5 Módulos Limitados 21

5.1 Módulos Induzidos . . . . . . . . . . . . . . . . . . . . . . . 21

5.2 Carácter dos Módulos Induzidos . . . . . . . . . . . . . . . . 23

5.3 Critério para Irredutibilidade . . . . . . . . . . . . . . . . . . 24

j.4 Chiral de de Rham . . . . . . . . . . . . . . . . . . . . 27

A Álgebras de Lie $\quad 31$

B Representações $\quad 37$

Referências Bibliográficas $\quad 39$ 


\section{Lista de Símbolos}

$\begin{array}{ll}V_{\lambda} & 3 \\ M(\lambda) & 4 \\ L(\lambda) & 4 \\ M(V) & 4 \\ \chi(V) & 5 \\ t^{r} & 8 \\ d_{a} & 8 \\ \mathfrak{h}_{\mathrm{Vect}\left(\mathbb{T}^{n}\right)} & 8 \\ T(W, \gamma) & 11 \\ q^{\gamma} \Omega^{k}\left(\mathbb{T}^{n}\right) & 12 \\ W(\gamma, k) & 12 \\ \left.\widehat{W}^{\gamma} \gamma, k\right) & 12 \\ M_{*} & 12 \\ Y(\cdot, z) & 15 \\ a(z)- & 16 \\ : a(z) b(z): & 16 \\ V_{\mathrm{Hyp}}^{+} & 17 \\ V_{\mathfrak{g l}} l_{n} & 18 \\ V_{\mathbb{Z}^{N}} & 19 \\ \mathcal{D} & 21 \\ M(T) & 21 \\ L(W, \gamma, h) & 23 \\ V_{\mathbb{Z}^{N}}^{k} & 28 \\ Q & 28 \\ \mathrm{~d} & 28\end{array}$


viii LISTA DE SÍMBOLOS 


\section{Introdução}

Recentemente, uma nova área da teoria das representações surgiu, a teoria de módulos limitados para álgebra de Lie de dimensão infinita com uma $\mathbb{Z}^{N}$-graduação densa. Para $N=1$ temos, como exemplo, as álgebras de Kac-Moody afim e a álgebra de Virasoro. Neste caso, a principal ferramenta para a teoria das representação é o conceito de módulo de Verma. No entanto, para $N>1$ não há maneira natural de se dividir $\mathbb{Z}^{N} \backslash\{0\}$ em partes positiva e negativa.

Considere a álgebra de polinômios de Laurent em $N$ variáveis complexas, $\mathbb{C}\left[t_{1}^{ \pm 1}, \ldots, t_{N}^{ \pm 1}\right]$. Sua álgebra de derivações, chamada de álgebra de Witt, que é isomorfa a álgebra de campos vetoriais em um toro $\mathrm{N}$-dimensional, Vect $\left(\mathbb{T}^{N}\right)$ é um exemplo de uma álgebra com uma $\mathbb{Z}^{N}$-graduação densa. Esta álgebra apresenta uma classe de módulos de origem geométrica, que decorre dos elementos da álgebra agirem em campos tensoriais como derivadas de Lie. Estes módulos, chamados de módulos tensoriais, são parametrizados a partir de módulos de $\mathfrak{g l}_{N}$ de dimensão finita.

Um teorema de $\mathrm{E}$. Rao garante que módulos irredutíveis de $\mathfrak{g l}_{N}$ dão origem a módulos tensoriais irredutíveis para $\operatorname{Vect}\left(\mathbb{T}^{N}\right)$, salvo quando estes pertencerem ao complexo de de Rham, os módulos de $k$-formas diferenciais. Mesmo estes sendo irredutíveis, seus módulos tensoriais podem não ser irredutíveis.

Para dividir esta álgebra em parte positiva e negativa consideramos uma coordenada especial, no nosso caso tomamos a coordenada $t_{0}$ da álgebra $\mathcal{D}=\operatorname{Vect}\left(\mathbb{T}^{N+1}\right)$. Para a decomposição triangular consideramos a $\mathbb{Z}$-graduação induzida pelo expoente da coordenada $t_{0}$. Podemos expandir a ação de $\operatorname{Vect}\left(\mathbb{T}^{N}\right)$ no módulo tensorial para a parte positiva e nula da decomposição triangular. Assim, podemos induzir a ação para toda álgebra, gerando uma nova classe de módulos

$$
M(T)=\operatorname{Ind}_{\mathcal{D}_{0} \oplus \mathcal{D}_{+}}^{\mathcal{D}} T .
$$

Estes módulos têm seus espaços de peso com dimensão infinita, porém, um teorema de S. Berman e Y. Billig [1] garantem que o quociente

$$
L(T)=M(T) / M^{r a d},
$$

onde $M^{\text {rad }}$ é o submódulo maximal, tem os espaços de peso com dimensão finita.

Temos então dois problemas com relação a estes módulos, determinar seu carácter e obter um critério de irredutibilidade. O caso $N=1$ foi estudado por Y. Billig, A Molev e R. Zhang [4]. No entanto o médoto aplicado por eles não funcionou para $N$ maiores.

Uma álgebra toroidal completa é o produto semidireto da extensão central da álgebra multiloop de uma álgebra de Lie pela álgebra de campos vetoriais em um toro. Um outro artigo de Y. Billig [2] apresenta uma categoria de módulos para álgebra toroidal completa utilizando álgebras de vértice. A partir desse artigo obtemos uma representação de campo livre para estes módulos, e a partir dela respondemos as duas perguntas.

Para o carácter devemos considerar a ação da subálgebra $\widetilde{\mathfrak{s l}}_{N+1} \subset \operatorname{Vect}\left(\mathbb{T}^{N+1}\right)$. Obtemos que o carácter coincide com um módulo de Verma generalizado para $\widetilde{\mathfrak{s l}}_{N+1}$, porém o módulo não é isomorfo a um módulo de Verma generalizado. Estes módulos foram chamados de módulos de Wakimoto generalizados, por Y. Billig e V. Futorny [3].

Para o problema de irredutibilidade um teorema de Y. Billig e V. Futorny [3] garante condições para certos módulos serem irredutíveis. Os módulos que este teorema não garante irredutibilidade 
aparecem em uma estrutura algébrica chamada Chiral de de Rham. A partir do operador diferencial deste Chiral temos que estes módulos são na verdade redutíveis.

\section{Organização do Trabalho}

No capítulo 1 apresentamos as definições de módulo de peso e de Verma, também apresentamos a definição e propriedades do carácter de um módulo. Estas definições serão amplamente utilizadas no texto.

No capítulo 2 apresentamos nosso objeto de estudo, a álgebra de campos vetoriais em um toro $N$-dimensional, Vect $\left(\mathbb{T}^{N}\right)$. Apresentamos uma motivação física, a álgebra de Virasoro, que é uma extensão central daquela álgebra. Por fim apresentamos a álgebra toroidal completa.

No capítulo 3 apresentamos um módulo para a álgebra Vect $\left(\mathbb{T}^{n}\right)$, o módulo tensorial $T(W, \gamma)$. Apresentamos um teorema que descreve quando os módulos tensoriais são irredutiveis. Nos casos em que o módulo tensorial não é irredutível, um teorema de Eswara Rao fornece muita informação sobre as componentes irredutíveis. Uma novidade que aparece neste texto são os seus caracteres que aparecem no final do capítulo.

No capítulo 4 é apresentada a estrutura de superálgebra de vértice e algumas de suas propriedades. Quatro dessas superálgebras são apresentadas, estas desempenham papel chave na representação de campos livres de $\operatorname{Vect}\left(\mathbb{T}^{N}\right)$.

Finalmente, no último capítulo são apresentados dois teoremas de Vyacheslav Futorny e Yuly Billig, o primeiro apresenta o carácter de módulos induzidos de $\operatorname{Vect}\left(\mathbb{T}^{N}\right)$ e o segundo expõe quais condições estes módulos devem satisfazer para serem irredutíveis. Por fim, apresentamos a estrutura do Chiral de de Rham.

Para compreensão do texto é recomendável uma certa familiaridade com álgebras de Lie e representações de estruturas algébricas. Não possuindo-a não se desencoraje, os apêndices apresentam toda informação necessária para acompanhar o texto para quem fez um curso básico de álgebra linear e saiba as propriedades básicas do produto tensorial. 


\section{Capítulo 1}

\section{Módulos de Peso e Carácter}

Neste capítulo são apresentadas as definições e algumas propriedades de módulo de peso e de caráter de módulo, seguindo [9], e também apresentamos as fórmulas dos caracteres dos módulos de Verma $U\left(\widetilde{\mathfrak{s}}_{N}^{-}\right)$e $U\left(\widehat{\mathfrak{g l}}_{N}^{-}\right)$que serão utilizadas mais adiante no texto.

\subsection{Módulos de peso}

Seja $\mathfrak{g}$ uma álgebra de Lie semi-simples de dimensão finita sobre um corpo $k$ de característica $0, \mathfrak{h}$ uma subálgebra de Cartan de $\mathfrak{g}, \Phi$ um sistema de raízes e $V$ um $\mathfrak{g}$-módulo de dimensão finita. Sabemos que se $V$ for de dimensão finita, então todo elemento de $\mathfrak{h}$ será diagonalizável. Como $\mathfrak{h}$ é abeliana, temos que os elementos de $\mathfrak{h}$ são simultaneamente diagonalizáveis. Assim, para todo $\lambda \in \mathfrak{h}^{*}$, podemos definir $V_{\lambda}:=\{v \in V \mid \forall h \in \mathfrak{h}, h . v=\lambda(h) v\}$ e teremos

$$
V=\bigoplus_{\lambda \in \mathfrak{h}^{*}} V_{\lambda}
$$

Quando $V_{\lambda} \neq 0$ dizemos que $V_{\lambda}$ é um espaço de peso e que $\lambda$ é um peso de $V$. Mesmo no caso de dimensão infinita, se $V$ for soma de espaços de peso, diremos que $V$ é um módulo de peso. Aqui vale observar que os $\lambda$ podem ser vistos como auto-valores generalizados.

Exemplo 1. A álgebra $\mathfrak{g}$ pode agir sobre si mesma pela representação adjunta, neste caso os pesos são as raízes de $\mathrm{g}$.

Para $\Phi$, seja $\Phi^{+}$uma escolha de raízes positivas, dizemos que (para esta escolha de raízes positivas) $v \neq 0$ é um vetor de peso máximo se para todo $\alpha \in \Pi^{+}, \mathfrak{g}_{\alpha} v=0$, onde $\mathfrak{g}_{\alpha}$ é o espaço de peso $\alpha$ da representação adjunta. No caso de um módulo ser gerado por um vetor de peso máximo, diremos que este módulo é de peso máximo. Além disso, podemos definir $\mathfrak{g}^{+}=\oplus_{\alpha \in \Pi^{+}} \mathfrak{g}_{\alpha} \mathrm{e}$ $\mathfrak{g}^{-}=\oplus_{\alpha \in \Pi^{-}} \mathfrak{g}_{\alpha}$ onde $\Phi^{-}=-\Phi^{+}$, e temos a seguinte decomposição $\mathfrak{g}=\mathfrak{g}^{-} \oplus \mathfrak{h} \oplus \mathfrak{g}^{+}$, que chamaremos de decomposição triangular de $\mathfrak{g}$.

Exemplo 2. Seja $\mathfrak{g}=\mathfrak{s l}_{2}$ e $k=\mathbb{C}$, e tome a seguinte base para $\mathfrak{g}$

$$
f=\left(\begin{array}{ll}
0 & 0 \\
1 & 0
\end{array}\right), \quad h=\left(\begin{array}{cc}
1 & 0 \\
0 & -1
\end{array}\right), \quad e=\left(\begin{array}{ll}
0 & 1 \\
0 & 0
\end{array}\right) .
$$

Tomemos a seguinte subálgebra de Cartan $\mathfrak{h}=\langle h\rangle=\mathbb{C} h$ e como parte positiva $\mathfrak{g}^{+}=\langle e\rangle=\mathbb{C} e$, e seja $V:=M(\lambda)$ o módulo gerado pelo vetor $v$ de peso máximo $\lambda$. Vamos mostrar como e e $f$ agem neste módulo. Temos que e.v $=0$ já que $v$ é de peso máximo, para $f$ temos

$$
h . f . v=f . h . v+[h, f] . v=\lambda f . v-2 f . v=(\lambda-2) f . v .
$$

Mais geralmente, seja $v_{0}=v$ e $v_{i}=f \cdot v_{i-1}$ temos

$$
\text { h.f. } v_{i}=f . h . v_{i}+[h, f] \cdot v_{i}=(\lambda-2 i) f \cdot v_{i}-2 f \cdot v_{i}=(\lambda-2(i+1)) f \cdot v_{i} .
$$


Quanto à ação de $e$ temos

$$
\text { e. } v_{1}=e . f . v_{0}=f . e \cdot v_{0}+[e, f] v_{0}=h \cdot v_{0}=\lambda v_{0} .
$$

Para $i>0$, temos

e. $v_{i}=e . f . v_{i-1}=f . e \cdot v_{i-1}+h v_{i-1}=$ f.e. $v_{i-1}+(\lambda-2(i-1)) v_{i-1}=\sum_{n=0}^{i-1}(\lambda-2 n) v_{i-1}=i(\lambda+1-i) v_{i-1}$.

Em resumo, temos o seguinte diagrama, onde cada ponto é um espaço de peso.

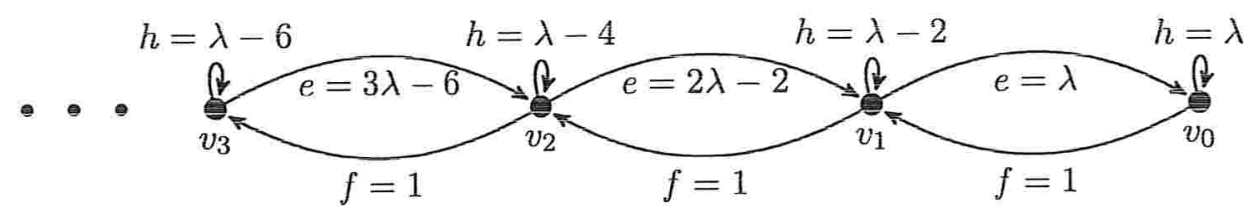

Podemos observar que se, e somente se, $\lambda$ for inteiro positivo, existirá $i$ tal que $i(\lambda+1-i)=0$, mais precisamente $i=\lambda+1$, ou seja, o espaço de peso $V_{-\lambda-2}\left(=\mathbb{C} v_{\lambda+1}\right)$ será anulado por e, disso segue que

$$
L:=\bigoplus_{n=-\lambda-1}^{-\infty} \mathbb{C} v_{n}
$$

é um submódulo de $V$. Este submódulo é o submódulo maximal de $M(\lambda)$ e assim definimos $L(\lambda):=$ $M(\lambda) / L$. Com isso, temos todos os módulos de dimensão finita de $\mathfrak{s t}_{2}$. Com efeito, dado um módulo de dimensão finita $n$, este será isomorfo a $L(n-1)$.

\subsection{Módulos de Verma}

Do último exemplo de seção anterior podemos ver que o módulo $M(\lambda)$ poderia ser definido como $U\left(\mathfrak{g}^{-}\right) v_{0}=\left\{y \cdot v_{0} \mid y \in U\left(\mathfrak{g}^{-}\right)\right\}$, onde $U\left(\mathfrak{g}^{-}\right)$é a álgebra universal envelopante de $\mathfrak{g}^{-}$. Isto nos leva a definição de módulo de Verma, nomeado em homenagem ao matemático Daya-Nand Verma.

Definição 1.2.1. Seja $\lambda \in \mathfrak{h}^{*}$, e seja $k_{\lambda}$ um módulo unidimensional para a sub-álgebra de Borel $\mathfrak{b}:=\mathfrak{h} \oplus \mathfrak{g}^{+}$, onde $h \in \mathfrak{h}$ age por multiplicação por $\lambda(h)$ e $\mathfrak{g}^{+}$age trivialmente. Definimos o módulo de Verma de $\mathfrak{g}$ de peso máximo $\lambda$ como sendo $M(\lambda)=U(\mathfrak{g}) \otimes_{U(\mathfrak{b})} k_{\lambda}$. Além disso, definimos $L(\lambda)=M(\lambda) / L$, onde $L$ é o submódulo maximal de $M(\lambda)$.

O Teorema PBW (veja B.0.11) implica que $M(\lambda)$ é isomorfo a $U\left(\mathfrak{g}^{-}\right) \otimes k_{\lambda}$.

Uma generalização que podemos fazer com os módulos de Verma é no lugar de fazermos o produto tensorial por módulo unidimensional $F_{\lambda}$ de $\mathfrak{b}$, podemos fazê-lo por um módulo qualquer (de dimensão finita) $V$ de uma sub-álgebra parabólica p , i. e., uma sub-álgebra que contém uma sub-álgebra de Borel.

Definição 1.2.2. Seja $V$ um módulo de dimensão finita para a sub-álgebra parabólica p. Definimos $M(V):=U(\mathfrak{g}) \otimes_{U(\mathfrak{b})} V$ como sendo o módulo de Verma generalizado.

Novamente por PBW temos $M(V) \simeq U\left(\mathfrak{g}^{-}\right) \otimes V$. A partir de agora iremos nos referir ao módulo de Verma generalizado pela sua sigla em inglês, GVM.

\subsection{Carácter}

Dado um módulo de peso $V$, uma outra maneira de descrevê-lo é através da dimensão dos seus espaços de pesos. Em $\mathbb{Z}\left[\left[\mathfrak{h}^{*}\right]\right]$, definimos, para cada $\lambda \in \mathfrak{h}^{*}$, o símbolo formal $e^{\lambda}$. Estes símbolos formais definem uma base de $\mathbb{Z}\left[\left[\mathfrak{h}^{*}\right]\right]$. Com isto estamos prontos para apresentar a definição de caráter de um módulo. 
Definição 1.3.1. Dado um módulo de peso $V$, a seguinte soma formal $\chi(V) \in \mathbb{Z}\left[\left[\mathfrak{h}^{*}\right]\right]$ é definida como sendo o carácter do módulo $V$.

$$
\chi(V):=\sum_{\lambda \in \mathfrak{h}^{*}} \operatorname{dim} V_{\lambda} e^{\lambda} .
$$

Exemplo 3. Seja $\mathfrak{g}=\mathfrak{s l}_{2}$ e considere $V=L(2)$. Temos que $V=V_{-2} \oplus V_{0} \oplus V_{2}$, cada um destes espaços de peso tem dimensão um. Assim, o caráter de $V$ é dado por

$$
\chi(V)=e^{-2}+e^{0}+e^{2} .
$$

Agora, se considerarmos $V=M(\lambda)$, para $\lambda$ qualquer, temos

$$
\chi(V)=\sum_{i \in \mathbb{Z}^{+}} e^{\lambda-2 i}=\frac{e^{\lambda}}{1-e^{-2}} .
$$

Proposição 1.3.2. Sejam g uma álgebra de Lie e $V$ e $W$ dois g-módulos, então temos que

$$
\chi(V \oplus W)=\chi(V)+\chi(W) \quad e \quad \chi(V \otimes W)=\chi(V) \chi(W) .
$$

Geralmente, o carácter de um módulo de Verma é representado pelo carácter da envelopante universal da parte negativa. Em particular, o caracteres dos módulos de Verma das álgebras $\widetilde{\mathfrak{s l}}_{N}=$ $\mathbb{C}\left[t^{ \pm 1}\right] \otimes \mathfrak{s l}_{N}$ e $\widehat{\mathfrak{g l}}_{N}=\mathbb{C}\left[t^{ \pm 1}\right] \otimes \mathfrak{g l}_{N} \oplus \mathbb{C} C$ são apresentado pela seguinte proposição:

Proposição 1.3.3. Temos que

$$
\chi\left(U\left(\tilde{\mathfrak{s l}}_{N}^{-}\right)\right)=\prod_{k=1}^{\infty}\left(1-s^{k}\right)^{-N^{2}+1} \text { e } \chi\left(U\left(\widehat{\mathfrak{g l}}_{N}^{-}\right)\right)=\prod_{k=1}^{\infty}\left(1-s^{k}\right)^{-N^{2}} .
$$

Demonstração. Obter estas fórmulas elegantes para os caracteres exige um conhecimento de fórmulas de caracteres, uma boa fonte que tem essas fórmulas é [12]. Aqui iremos mostrar como calcular a dimensão de um dado peso para um modulo de Verma para a álgebra $\tilde{\mathfrak{s l}}_{N}^{-}$(Para ver as estruturas das raízes veja o Apêndice $A$ ).

Por exemplo, tome $\mu=\lambda-3 \alpha_{1}-2 \alpha_{2}$ Vamos calcular a dimensão de $V_{\mu}$ em $M(\lambda)$. Témos as seguintes maneiras de chegarmos a $\mu$ partindo de $\lambda$ utilizando as raízes:

- $-\alpha_{1}-\alpha_{1}-\alpha_{1}-\alpha_{2}-\alpha_{2}$

- $-\alpha_{1}-\alpha_{1}-\left(\alpha_{1}+\alpha_{2}\right)-\alpha_{2}$

- $-\alpha_{1}-\left(\alpha_{1}+\alpha_{2}\right)-\left(\alpha_{1}+\alpha_{2}\right)$

- $-\alpha_{1}-\left(2 \alpha_{1}+\alpha_{2}\right)-\alpha_{2}$

- $-\left(2 \alpha_{1}+\alpha_{2}\right)-\left(\alpha_{1}+\alpha_{2}\right)$

- $-\alpha_{1}-\alpha_{1}-\left(\alpha_{1}+2 \alpha_{2}\right)$

- $-\left(3 \alpha_{1}+2 \alpha_{2}\right)$

Logo, $\operatorname{dim}\left(V_{\mu}\right)=7$. 


\section{Capítulo 2}

\section{Álgebra Vect $\left(\mathbb{T}^{N}\right)$}

Neste capítulo apresentamos inicialmente a álgebra de Witt, que coincide com a álgebra de campos vetoriais em um círculo. Em seguida apresentamos a definição de extensão central e como exemplo a álgebra de Virasoro, extensão central da álgebra de Witt. Aqui apresentamos nosso objeto de estudo, uma generalização da álgebra de Witt, a álgebra de campos vetoriais em um toro, $\operatorname{Vect}\left(\mathbb{T}^{N}\right)$. Por fim, apresentamos uma álgebra que será importante mais adiante, a álgebra toroidal completa.

\section{$2.1 \quad$ Ảlgebra de Witt}

A álgebra de Witt $W$, nomeada em homenagem ao matemático Ernest Witt, é a álgebra de derivações do anel $\mathbb{C}\left[z^{ \pm 1}\right]$. Definimos, para $n, m \in \mathbb{Z}$

$$
L_{n}:=-z^{n+1} \frac{\partial}{\partial z} \quad \text { e } \quad\left[L_{m}, L_{n}\right]:=(m-n) L_{m+n} .
$$

No apêndice A mostramos que os $L_{n}$ formam uma base de $W$.

Uma outra maneira de enxergar esta álgebra é pelo seu carácter geométrico. Usualmente o conjunto de campo de vetores de uma variedade $M$ é denotado por $\mathfrak{X}(M)$, no entanto, estamos interessados nos campos que sejam analíticos, i. e., campos em que suas coordenadas sejam funções analíticas. Este conjunto de campos analíticos tem uma estrutura natural de álgebra de Lie, que denotaremos por $\operatorname{Vect}(M)$. Esta estrutura decorre do fato de campos vetoriais serem uma derivada, mais explicitamente, sejam $X, Y \in \operatorname{Vect}(M)$ e $h: M \rightarrow k$ analítica, definimos

$$
[X, Y](h)=X(Y(h))-Y(X(h)) .
$$

Neste caso, como era para ser, o colchete é bilinear para escalares mas não para funções. Para ver como uma função pode ser retirada do colchete, devemos lembrar da regra de Leibniz da derivada do produto. Sejam $f, g: M \rightarrow k$ analíticas, temos que

$$
\begin{gathered}
{[f X, g Y](h)=f X(g Y(h))-g Y(f X(h))=f X(g) Y(h)+f g X(Y(h)-g Y(f) X(h)-g f Y(X(h)) \Rightarrow} \\
\Rightarrow[f X, g Y]=f g[X, Y]+f X(g) Y-g Y(f) X .
\end{gathered}
$$

Para $M=\mathbb{S}^{1}$, o círculo unitário, considere a coordenada $\theta$. Definimos

$$
L_{n}:=-\theta^{n+1} \frac{\partial}{\partial \theta}
$$

e é fácil ver que os $L_{n}$ formam uma base para $\operatorname{Vect}\left(\mathbb{S}^{1}\right)$. Seu colchete é dado por

$$
\left[L_{m}, L_{n}\right]=\left[\theta^{m+1} \frac{\partial}{\partial \theta}, \theta^{n+1} \frac{\partial}{\partial \theta}\right]=\theta^{m+n+2}\left[\frac{\partial}{\partial \theta}, \frac{\partial}{\partial \theta}\right]+(n+1) \theta^{m+n+1} \frac{\partial}{\partial \theta}-(m+1) \theta^{m+n+1} \frac{\partial}{\partial \theta}=
$$




$$
=(m-n) L_{m+n}
$$

\subsection{Extensões Centrais e Generalização da Âlgebra de Witt}

Definição 2.2.1. Diremos que $\dot{\mathfrak{g}}$ é uma extensão central de $\mathfrak{g}$ se existir uma sequencia exata curta

$$
0 \longrightarrow \mathfrak{z} \longrightarrow \dot{\mathfrak{g}} \longrightarrow \mathfrak{g} \longrightarrow 0
$$

com a imagem de $z$ coincidindo com o centro de $\dot{\mathfrak{g}}$.

A álgebra de Virasoro é uma extensão central da álgebra de Witt. Ela é gerada por $\left\{L_{n}, n \in\right.$ $\mathbb{Z}\} \cup\{c\}$ e as seguintes relações

$$
\left[L_{m}, L_{n}\right]=(m-n) L_{m+n}+\frac{c}{12}\left(m^{3}-m\right) \delta_{m+n, 0},\left[L_{n}, c\right]=0
$$

Apesar de ser primeiro descrita como extensão central da álgebra de Witt, a álgebra de Virasoro tem seu nome em homenagem ao físico Miguel Ángel Virasoro, pois este apresentou operadores do seu estudo de modelos de ressonâncias duais. Está álgebra ainda hoje tem mostrado várias aplicações na física.

Uma generalização natural de álgebra de Witt é considerar o anel $A_{N}:=\mathbb{C}\left[t_{1}^{ \pm 1}, t_{2}^{ \pm 1}, \ldots, t_{N}^{ \pm 1}\right]$. Definimos como a álgebra de Witt, $W^{N}$, a álgebra de derivação de $A_{N}$, simbolicamente, $W^{N}:=$ $\operatorname{Der} A^{N}$. Seja $\mathbb{T}^{N}=\mathbb{S}^{1} \times \ldots \times \mathbb{S}^{1}$, o toro $N$-dimensional, podemos considerar $W^{N} \simeq \operatorname{Vect}\left(\mathbb{T}^{N}\right)$. De fato, pelo teorema de Schwarz temos que o colchete $\left[\partial / \partial t_{i}, \partial / \partial t_{j}\right]=0$ para $i, j \in\{1, \ldots, N\}$.

Uma notação que será muito útil é a notação de multi-índice, que é definida da seguinte forma, dado $r \in \mathbb{Z}^{n}$ então $t^{r}:=t_{1}^{r_{1}} t_{2}^{r_{2}} \ldots t_{n}^{r_{n}}$. Além disso, definimos $d_{a}:=t_{a} \partial / \partial t_{a}$ e é fácil ver que $\left\{t^{r} d_{a} \mid r \in \mathbb{Z}^{n}, a \in\{1, \ldots, n\}\right\}$ é uma base $\operatorname{de} \operatorname{Vect}\left(\mathbb{T}^{N}\right)$.

Com isso, o colchete em $\operatorname{Vect}\left(\mathbb{T}^{N}\right)$ é dado por, sejam $a, b \in\{1, \ldots, N\}$ e $r, m \in \mathbb{Z}^{N}$,

$$
\left[t^{r} d_{a}, t^{m} d_{b}\right]=t^{r+m}\left[d_{a}, d_{b}\right]+m_{b} t^{r+m} d_{b}-r_{a} t^{r+m} d_{a}=m_{b} t^{r+m} d_{b}-r_{a} t^{r+m} d_{a}
$$

Em Vect $\left(\mathbb{T}^{N}\right)$ definimos a seguinte $\operatorname{CSA}, \mathfrak{h}_{\text {Vect }\left(\mathbb{T}^{N}\right)}:=\left\langle d_{1}, \ldots, d_{N}\right\rangle$. Uma propriedade que será de grande importância é que Vect $\left(\mathbb{T}^{N}\right)$ contém uma cópia de $\mathfrak{s l}_{N+1}$. De fato, temos a seguinte inclusão para $i, j \in\{1, \ldots, N\}, E_{i j} \rightarrow t_{i} t_{j}^{-1} d_{j}, E_{i, N+1} \rightarrow-t_{i} D, E_{N+1, j} \rightarrow t_{j}^{-1} d_{j}$ e $E_{N+1, N+1} \rightarrow-D$, onde $D:=\sum_{k=1}^{N} d_{k}$ e $E^{i j}:=\left(\delta_{i k} \delta_{j l}\right)_{k, l} \in \mathfrak{g l}_{N}$. Álém disso, se considerarmos $\operatorname{Vect}\left(\mathbb{T}^{n+1}\right):=$ $\oplus_{p=0}^{n} \mathbb{C}\left[t_{0}^{ \pm 1}, t_{1}^{ \pm 1}, \ldots, t_{N}^{ \pm 1}\right] d_{p}$, fica claro que $\widetilde{\mathfrak{s l}}_{N+1}:=\mathbb{C}\left[t_{0}^{ \pm 1}\right] \otimes \mathfrak{s l}_{N+1} \subset \operatorname{Vect}\left(\mathbb{T}^{N}\right)$. Este fato será importante mais adiante.

\section{3 Álgebra Toroidal}

Álgebras toroidais são, de uma maneira bem natural, generalizações de álgebras de Kac-Moody afim.

Sejam $\dot{\mathfrak{g}}$ uma álgebra de Lie simples sobre $\mathbb{C}$ e $N>1 \mathrm{um}$ inteiro. Consideremos a álgebra de Lie $\mathcal{R} \otimes \dot{\mathfrak{g}}$ de aplicações de um toro $N$ dimensional em $\dot{\mathfrak{g}}$, onde $\mathcal{R}=\mathbb{C}\left[t_{0}^{ \pm}, t_{1}^{ \pm}, \ldots, t_{N}^{ \pm}\right]$é a álgebra de polinômios de Fourier sobre um toro. A extensão central universal desta álgebra é devida a Kassel, sua construção é dada a seguir.

Seja $\Omega_{\mathcal{R}}^{1}\left(\mathbb{T}^{N}\right)$ o espaço das 1-formas diferenciais em um toro: $\Omega_{\mathcal{R}}^{1}\left(\mathbb{T}^{N}\right)=\oplus_{p=0}^{N} \mathcal{R} d t_{\bar{p}}$. Podemos escolher a seguinte base para $\Omega_{\mathcal{R}}^{1}\left(\mathbb{T}^{N}\right)$, como um $\mathcal{R}$-módulo, $\left\{k_{a}=t_{a}^{-1} d t_{a} \mid a=0, \ldots, N\right\}$. A derivada exterior é uma aplicação do espaço $\mathcal{R}$ em $\Omega_{\mathcal{R}}^{1}\left(\mathbb{T}^{N}\right)$ definida como $\mathrm{d}(f)=\sum_{p=0}^{N}{ }_{p}\left(\partial f / \partial t_{p}\right) k_{p}$. O centro $\mathcal{K}$ da extensão universal central $\dot{\mathfrak{g}} \otimes \mathcal{R} \oplus \mathcal{K}$ é realizado como

$$
\mathcal{K}=\Omega_{\mathcal{R}}^{1}\left(\mathbb{T}^{N}\right) / \mathrm{d}(\mathcal{R})
$$


e o colchete de Lie é dado por

$$
\left[f_{1}(t) \otimes g_{1}, f_{2}(t) \otimes g_{2}\right]=f_{1} f_{2} \otimes\left[g_{1}, g_{2}\right]+\left(g_{1} \mid g_{2}\right) \overline{f_{2} d f_{1}},
$$

onde $g_{1}, g_{2} \in \dot{\mathfrak{g}}, f_{1}, f_{2} \in \mathcal{R},(\cdot \mid \cdot)$ é a forma de Killing de $\dot{\mathfrak{g}}$ e a barra em $\overline{f_{2} d f_{1}}$ indica a imagem pela projeção canônica $\Omega_{\mathcal{R}}^{1}\left(\mathbb{T}^{N}\right) \rightarrow \mathcal{K}$, no que se segue omitiremos esta barra para representar elementos de $\mathcal{K}$.

A ação natural de $\mathcal{D}=\operatorname{Vect}\left(\mathbb{T}^{N}\right)$ em $\mathcal{R} \otimes \dot{\mathfrak{g}}$ é dada por

$$
\left[t^{r} d_{a}, t^{m} g\right]=m_{a} t^{r+m} g
$$

esta ação é unicamente estendida a $\mathcal{K}$

$$
\left[t^{r} d_{a}, t_{b}^{k}\right]=m_{a} t^{r+m} k_{b}+\delta_{a b} \sum_{p=0}^{N} r_{p} t^{r+m} k_{p} .
$$

A álgebra toroidal completa é o produto semidireto

$$
\mathfrak{g}=(\mathcal{R} \otimes \dot{\mathfrak{g}} \oplus \mathcal{K}) \rtimes \mathcal{D} .
$$

Em [2] foi classificada uma categoria de módulos para a álgebra toroidal completa, tal categoria admite o seguinte caso particular $\dot{\mathfrak{g}}=0$. Neste caso obtemos módulos para a álgebra $\mathcal{K} \rtimes \mathcal{D}$. A técnica que utilizaremos será restringir estes módulos a ação de $\mathcal{D}$, o que veremos a seguir é que a maior parte destes módulos permanece irredutível quando restritos a ação de $\mathcal{D}$. 


\section{Capítulo 3}

\section{Módulo Tensorial}

Neste capítulo apresentamos o módulo tensorial como definido em [3]. Toda álgebra de Lie será sobre o corpo $\mathbb{C}$.

\subsection{Módulo Tensorial}

O módulo tensorial é um módulo para a álgebra $\operatorname{Vect}\left(\mathbb{T}^{N}\right)$ e é definido da seguinte forma.

Definição 3.1.1. Sejam $W$ um $\mathfrak{g l}_{N}$-módulo de dimensão finita e $\gamma \in \mathbb{C}^{N}$, então o espaço vetorial

$$
T(W, \gamma):=q^{\gamma} \mathbb{C}\left[q_{1}^{ \pm 1}, \ldots, q_{N}^{ \pm 1}\right] \otimes W
$$

é um $\operatorname{Vect}\left(\mathbb{T}^{N}\right)$-módulo com a ação dada por

$$
t^{r} d_{a}\left(q^{\mu} \otimes w\right):=\mu_{a} q^{\mu+r} \otimes w+\sum_{p=1}^{N} r_{p} q^{\mu+r} \otimes E^{p a} w,
$$

onde $r \in \mathbb{Z}^{N}, \mu \in \gamma+\mathbb{Z}^{N}, w \in W, a \in\{1, \ldots, N\}$ e $E^{p a}=\left(\delta_{p i} \delta_{a j}\right)_{i, j} \in \mathfrak{g l}_{N}$.

Vamos verificar que, de fato, $T(W, \gamma)$ é um $\operatorname{Vect}\left(\mathbb{T}^{N}\right)$-módulo. Temos por um lado

$$
\begin{gathered}
{\left[t^{r} d_{a}, t^{s} d_{b}\right]\left(q^{\mu} \otimes w\right)=\left(s_{a} t^{r+s} d_{b}-r_{b} t^{r+s} d_{a}\right)\left(q^{\mu} \otimes w\right)=} \\
=\left(s_{a} \mu_{b}-r_{b} \mu_{a}\right) q^{\mu+r+s} \otimes w+\sum_{p=1}^{N}(r+s)_{p}\left(s_{a}-r_{b}\right) q^{\mu+r+s} \otimes\left(E^{p b}-E^{p a}\right) w
\end{gathered}
$$

Por outro lado

$$
\begin{gathered}
t^{r} d_{a}\left(t^{s} d_{b}\left(q^{\mu} \otimes w\right)\right)=t^{r} d_{a}\left(\mu_{b} q^{\mu+s} \otimes w+\sum_{p=1}^{N} s_{p} q^{\mu+s} \otimes E^{p b} w\right)= \\
=\mu_{b}(\mu+s)_{a} q^{\mu+r+s} \otimes w+\sum_{p=1}^{N} \mu_{b} r_{p} q^{\mu+r+s} \otimes E^{p a} w+ \\
+\sum_{p=1}^{N} s_{p}(\mu+s)_{a} q^{\mu+r+s} \otimes E^{p b} w+\sum_{p=1}^{N} s_{a} r_{p} q^{\mu+r+s} \otimes E^{p b} w
\end{gathered}
$$

Observe que $\sum_{p} r_{p} E^{p a} \mu=\mu_{a} r$. Por simetria temos

$$
\begin{gathered}
t^{s} d_{b}\left(t^{r} d_{a}\left(q^{\mu} \otimes w\right)\right)=\mu_{a}(\mu+r)_{b} q^{\mu+r+s} \otimes w+\sum_{p=1}^{N} \mu_{a} s_{p} q^{\mu+r+s} \otimes E^{p b} w+ \\
+\sum_{p=1}^{N}\left((r+s)_{p} r_{b}+r_{p} \mu_{b}\right) q^{\mu+r+s} \otimes E^{p a} w
\end{gathered}
$$

Assim

$$
t^{r} d_{a}\left(t^{s} d_{b}\left(q^{\mu} \otimes w\right)\right)-t^{s} d_{b}\left(t^{r} d_{a}\left(q^{\mu} \otimes w\right)\right)=\left[t^{r} d_{a}, t^{s} d_{b}\right]\left(q^{\mu} \otimes w\right)
$$

Portanto, $T(W ; \gamma)$ é um $\operatorname{Vect}\left(\mathbb{T}^{N}\right)$-módulo 


\subsection{Complexo de de Rham}

A álgebra exterior $\bigwedge^{k}\left(\mathbb{C}^{N}\right)$ é um famoso $\mathfrak{g l}^{N}$-módulo com a ação dada por

$$
X\left(v_{1} \wedge \ldots \wedge v_{k}\right)=\sum_{i=1}^{k} v_{1} \wedge \ldots v_{i-1} \wedge X v_{i} \wedge v_{i+1} \ldots \wedge v_{k}
$$

onde $X \in \mathfrak{g l}^{N}$ e $v_{j} \in \mathbb{C}^{N}, \forall j \in\{1, \ldots, k\}$. Seu módulo tensorial pode ser identificado como formas diferenciais via os seguintes isomorfismos

$$
q^{\mu} \otimes e_{i_{1}} \wedge \ldots \wedge e_{i_{k}} \rightarrow t^{\mu} t_{i_{1}}^{-1} d t_{i_{1}} \wedge \ldots \wedge t_{i_{k}}^{-1} d t_{i_{k}}
$$

assim, chamaremos estes de módulos tensoriais do complexo de de Rham,

$$
q^{\gamma} \Omega^{k}\left(\mathbb{T}^{N}\right):=T\left(\bigwedge^{k}\left(\mathbb{C}^{N}\right), \gamma\right)
$$

para $k \in\{1, \ldots, N-1\}$, e para $k \in\{0, N\}$ definimos os módulos tensoriais com $W$ sendo os $\mathfrak{g l}_{N}$-módulos onde a identidade age multiplicando por $k$, e a subálgebra $\mathfrak{s l}_{N}$ age trivialmente. $\mathrm{O}$ interessante é que a derivada exterior das formas diferencias é um morfismo de $\operatorname{Vect}\left(\mathbb{T}^{N}\right)$-módulos e, como já sabemos, a imagem pela derivada exterior nem sempre é todo espaço seguinte do complexo. Isso já nos mostra que estes módulos tensoriais não são irredutíveis, porêm, um teorema presente em [16] mostra que estes são exatamente os únicos redutíveis.

Teorema 3.2.1. Seja $W$ um $\mathfrak{g l}_{N}$-módulo irredutivel de dimensão finita, e seja $\gamma \in \mathbb{C}^{N}$, então o módulo tensorial $T(W, \gamma)$ será redutivel se ele aparecer no complexo de de Rham

$$
q^{\gamma} \Omega^{0}\left(\mathbb{T}^{N}\right) \stackrel{d}{\rightarrow} q^{\gamma} \Omega^{1}\left(\mathbb{T}^{N}\right) \stackrel{d}{\rightarrow} \ldots \stackrel{d}{\rightarrow} q^{\gamma} \Omega^{N}\left(\mathbb{T}^{N}\right)
$$

a menos dos termos extremos $(k=0, N)$ quando $\gamma \notin \mathbb{Z}^{N}$, estes e todos os demais serão irredutíveis.

\subsection{Um Teorema de Rao}

O seguinte teorema, devido a Eswara Rao, apareceu em [16], no entanto, seguiremos [7], pois este apresenta uma prova mais simples deste teorema. Inicialmente observe que se $\gamma \in \mathbb{Z}^{N}$, então $T(W, \gamma)=T(W, 0)$. Com isso definimos os seguintes subespaços de $q^{\gamma} \Omega^{k}\left(\mathbb{T}^{N}\right)$ para $k \in\{1, \ldots, N-$ $1\}$

$$
W(\gamma, k):=\bigoplus_{\mu \in \gamma+\mathbb{Z}^{N}} q^{\mu} \otimes\left(\mathbb{C}(\mu) \wedge \mathbb{C}^{N} \wedge \ldots \wedge \mathbb{C}^{N}\right) .
$$

E quando $\gamma=0$ definimos

$$
\widehat{W}(0, k):=W(0, k) \oplus q^{0} \otimes\left(\mathbb{C}^{N} \wedge \ldots \wedge \mathbb{C}^{N}\right) .
$$

Para $\gamma \neq 0$ também denotaremos $\widehat{W}(\gamma, k)=W(\gamma, k)$, por conveniência.

Dado um submódulo $M$ de $T(W, \gamma)$, definimos $M_{\mu}:=\left\{v \in W \mid q^{\mu} \& v \in M\right\}$ e $M_{*}:=\cap_{\mu \in \gamma+\mathbb{Z}^{N}} M_{\mu}$. O seguinte lema será utilizado no teorema de Rao.

Lema 3.3.1. Se $M_{*} \neq\{0\}$, então $M=T(W, \gamma)$.

Demonstração. Suponha $M_{*} \neq\{0\}$ e seja $w \neq 0 \in M_{*}$, temos que para todo $a \in\{1, \ldots, N\}$ e $r \in \mathbb{Z}^{N}$,

$$
t^{r} d_{a} q^{\mu-r} \otimes w=(\mu-r)_{a} q^{\mu} \otimes w+\sum_{p=1}^{k} r_{p} q^{\mu} \otimes E^{p a} w \Rightarrow E^{i j} w \in M_{\mu}
$$


para todo $i, j \in\{1, \ldots, N\}$ e $\mu \in \gamma+\mathbb{Z}^{N}$. Com isto temos que $M_{*}$ é um $g l_{N}$-submódulo de $W$, porém $W$ é irredutível, logo $M_{*}=W$ e, portanto, $M=T(W, \gamma)$.

Teorema 3.3.2. Para $k \in\{1, \ldots, N-1\}$ temos que $W(\gamma, k)$ e $q^{\gamma} \Omega^{k}\left(\mathbb{T}^{N}\right) / \widehat{W}(\gamma, k) \operatorname{sano} \operatorname{Vect}\left(\mathbb{T}^{N}\right)$ módulos irredutiveis.

Demonstração. Considere a seguinte aplicação

$$
\begin{gathered}
\iota_{k}: q^{\gamma} \Omega^{k}\left(\mathbb{T}^{N}\right) \rightarrow q^{\gamma} \Omega^{k+1}\left(\mathbb{T}^{N}\right) \\
q^{\mu} \otimes\left(v_{1} \wedge v_{2} \wedge \ldots \wedge v_{k}\right) \rightarrow q^{\mu} \otimes\left(\mu \wedge v_{1} \wedge v_{2} \wedge \ldots \wedge v_{k}\right) .
\end{gathered}
$$

Temos que $\iota_{k}$ é um morfismo de $\operatorname{Vect}\left(\mathbb{T}^{N}\right)$-módulos. Com efeito, seja $r \in \mathbb{Z}^{N}$ e $a \in\{1, \ldots, N\}$ temos

$$
\begin{gathered}
\iota_{k}\left(t^{r} d_{a}\left(q^{\mu} \otimes\left(v_{1} \wedge \ldots \wedge v_{k}\right)\right)=\mu_{a} q^{\mu+r} \otimes\left((\mu+r) \wedge v_{1} \wedge \ldots \wedge v_{k}\right)+\right. \\
+\sum_{p=1}^{N} \sum_{i=1}^{k} r_{p} q^{\mu+r} \otimes\left((\mu+r) \wedge v_{1} \wedge \ldots \wedge E^{p a}\left(v_{i}\right) \wedge \ldots \wedge v_{k}\right)= \\
=\mu_{a} q^{\mu+r} \otimes\left(\mu \wedge v_{1} \wedge \ldots \wedge v_{k}\right)+\sum_{p=1}^{N} r_{p} q^{\mu+r} \otimes\left(E^{p a} \mu \wedge v_{1} \wedge \ldots \wedge v_{k}\right)+ \\
+\sum_{p=1}^{N} \sum_{i=1}^{k} r_{p} q^{\mu+r} \otimes\left(\mu \wedge v_{1} \wedge \ldots \wedge E^{p a}\left(v_{i}\right) \wedge \ldots \wedge v_{k}\right)+ \\
+\sum_{p=1}^{N} \sum_{i=1}^{k} r_{p} q^{\mu+r} \otimes\left(r \wedge v_{1} \wedge \ldots \wedge E^{p a}\left(v_{i}\right) \wedge \ldots \wedge v_{k}\right)=t^{r} d_{a}\left(\iota_{k}\left(q^{\mu} \otimes\left(v_{1} \wedge \ldots \wedge v_{k}\right)\right)\right.
\end{gathered}
$$

pois para todo $i$

$$
\sum_{p=1}^{N} r_{p}\left(r \wedge v_{1} \wedge \ldots \wedge E^{p a}\left(v_{i}\right) \wedge \ldots \wedge v_{k}\right)=\left(r \wedge v_{1} \wedge \ldots \wedge r\left(v_{i}\right)_{a} \wedge \ldots \wedge v_{k}\right)=0
$$

onde $\left(v_{i}\right)_{a}$ é a $a$-ésima coordenada do vetor $v_{i}$. É fácil ver que

$$
\operatorname{ker}\left(\iota_{k}\right)=\widehat{W}(\gamma, k), \operatorname{im}\left(\iota_{k}\right)=W(\gamma, k+1),
$$

e pelo primeiro teorema do isomorfismo

$$
q^{\gamma} \Omega^{k}\left(\mathbb{T}^{N}\right) / \widehat{W}(\gamma, k) \simeq W(\gamma, k+1) .
$$

Observe que $W(\gamma, 1)=\oplus_{\mu \in \gamma+\mathbb{Z}^{N}} \mathbb{C}\left(q^{\mu} \otimes \mu\right)$ e $t^{r} d_{a}\left(q^{\mu} \otimes \mu\right)=\mu_{a}\left(q^{\mu+r} \otimes(\mu+r)\right)$, para todo $a \in\{1, \ldots, N\}$ e $r \in \mathbb{Z}^{N}$, logo $W(\gamma, 1)$ é irredutível. Além disso $\widehat{W}(\gamma, N)=q^{\gamma} \Omega^{N}\left(\mathbb{T}^{N}\right)$. Dessa forma, para provar o teorema só precisamos provar que $q^{\gamma} \Omega^{k}\left(\mathbb{T}^{N}\right) / \widehat{W}(\gamma, k)$ são irredutíveis para $k \in\{1, \ldots, N-1\}$. Para isso vamos introduzir a seguinte notação, seja $u=\left(u_{1}, u_{2}, \ldots, u_{N}\right) \in \mathbb{C}^{N}$ e $r \in \mathbb{Z}^{N}$ definimos

$$
D(u, r):=t^{r} \sum_{i=1}^{N} u_{i} d_{i}
$$

com esta notação a ação de $\operatorname{Vect}\left(\mathbb{T}^{N}\right)$ em $T(W, \gamma)$ fica

$$
D(u, r)\left(q^{\mu} \otimes w\right)=\langle u, \mu\rangle q^{\mu+r} \otimes w+q^{\mu+r} \otimes\left(r u^{t}\right) w,
$$

onde $\langle.,$.$\rangle é o produto interno usual e \left(r u^{t}\right) \in \mathfrak{g l}_{N}$. Aqui vale a pena notar que $\left(r u^{t}\right) w=\langle u, w\rangle \cdot r$ para todo $u, r, w \in \mathbb{C}^{N}$. 
Suponha que exista $M$ um sub-módulo de $q^{\gamma} \Omega^{k}$ tal que $\widehat{W}(\gamma, k)$ seja um submódulo próprio de $M$. Seja $q^{\mu} \otimes\left(v_{1} \wedge \ldots \wedge v_{k}\right) \in M \backslash \widehat{W}(\gamma, k)$. Temos que o conjunto $\left\{\mu, v_{1}, v_{2}, \ldots, v_{k}\right\}$ é 1.i.. Com efeito, sabemos que $\left\{v_{1}, v_{2}, \ldots, v_{k}\right\}$ é linearmente independente, logo suponha que existam $\alpha_{i} \in \mathbb{C}$ tais que $\mu=\sum_{i=1}^{k} \alpha_{i} v_{i}$, e sem perda de generalidade podemos supor $\alpha_{1} \neq 0$. Assim

$$
\widehat{M} \backslash \widehat{W}(\gamma, k) \ni \alpha_{1} q^{\mu} \otimes\left(v_{1} \wedge \ldots \wedge v_{k}\right)=\sum_{i=1}^{k} q^{\mu} \otimes\left(\alpha_{i} v_{i} \wedge \ldots \wedge v_{k}\right)=q^{\mu} \otimes\left(\mu \wedge v_{2} \wedge \ldots \wedge v_{k}\right)
$$

Absurdo, portanto, o conjunto $\left\{\mu, v_{1}, v_{2}, \ldots, v_{k}\right\}$ é linearmente independente, e assim, podemos escolher $u \in \mathbb{C}^{N}$ tal que $\langle u, \mu\rangle \neq 0$ e para todo $i,\left\langle u, v_{i}\right\rangle=0$, e teremos que para todo $r \in \mathbb{Z}^{N}$

$$
D(u, r) q^{\mu} \otimes\left(v_{1} \wedge \ldots \wedge v_{k}\right)=\langle u, \mu\rangle q^{\mu+r} \otimes\left(v_{1} \wedge \ldots \wedge v_{k}\right) \in M .
$$

Logo $\left(v_{1} \wedge \ldots \wedge v_{k}\right) \in M_{*}$ e pelo lema 3.3 .1 temos que $M=q^{\gamma} \Omega\left(\mathbb{T}^{N}\right)$, implicando que $\widehat{W}(\gamma, k)$ é maximal.

\subsection{Carácteres dos Submódulos}

Antes de descrevermos os carácteres dos submódulos, trataremos o caso $\gamma=0$ que é quando $\widehat{W}(0, k) \neq W(0, k)$. Temos que no quociente, $\widehat{W}(0, k) / W(0, k)$, Vect $\mathbb{T}^{N}$ age trivialmente. Com efeito, $t^{r} d_{a}\left(q^{0} \otimes\left(v_{1} \wedge \ldots \wedge v_{k}\right)\right) \in W(0, k)$ se $r \neq 0$. E se $r=0$ então $d_{a}\left(q^{0} \otimes\left(v_{1} \wedge \ldots \wedge v_{k}\right)\right)=0$. Com isso, temos que o único submódulo irredutível de $q^{\gamma} \Omega^{k}\left(\mathbb{T}^{N}\right)$ é, de fato, $W(\gamma, k)$. Assim concluímos com o seguinte teorema.

Teorema 3.4.1. Para $k \in\{1, \ldots, N-1\}$, o único sub-módulo próprio de $q^{\gamma} \Omega^{k}\left(\mathbb{T}^{N}\right)$ é $W(\gamma, k) e$ seu carácter é dado por

$$
\chi(W(\gamma, k))=\sum_{\substack{\mu \in \gamma+\mathbb{Z}^{N} \\
\mu \neq 0}}\left(\begin{array}{l}
n-1 \\
k-1
\end{array}\right) e^{\mu}
$$

onde, para $\mu \in \gamma+\mathbb{Z}^{N}$, definimos $\mu \in \mathfrak{h}_{\mathrm{Vect}\left(\mathbb{T}^{N}\right)}^{*}$ como $\mu\left(d_{a}\right)=\mu_{a}$.

Demonstração. Dado $\mu \in \gamma+\mathbb{Z}^{N}$ temos, como definido no enunciado do teorema o peso $\mu \in$ $\mathfrak{h}_{\mathrm{Vect}\left(\mathbb{T}^{N}\right)}^{*}$, e o espaço de peso $V_{\mu}$ é dado por

$$
V_{\mu}=q^{\mu} \otimes\left(\mathbb{C}(\mu) \wedge \mathbb{C}^{N} \wedge \ldots \wedge \mathbb{C}^{N}\right) .
$$

Se $\mu=0$ o espaço de peso $V_{\mu}$ tem dimensão zero, caso contrário, podemos, a partir de $\mu$, completar uma base de $\mathbb{C}^{N}$, digamos $\left\{\mu, v_{1}, \ldots, v_{N-1}\right\}$. E a partir desta base, podemos construir a seguinte base de $V_{\mu},\left\{\mu \wedge v_{i_{1}} \wedge \ldots \wedge v_{i_{k-1}} \mid 1 \leq i_{1}<i_{2}<\ldots<i_{k-1} \leq n-1\right\}$. Logo, concluímos que a dimensão de $V_{\mu}$ é $\left(\begin{array}{l}n-1 \\ k-1\end{array}\right)$. 


\title{
Representações da álgebra de Lie de campos vetoriais sobre um toro $\mathrm{N}$-dimensional
}

André Eduardo Zaidan

\author{
DISSERTAÇÃO APRESENTADA \\ $\mathrm{AO}$ \\ Instituto de Matemática E Estatística \\ DA \\ Universidade DE SÃo Paulo \\ PARA \\ OBTENÇÃO DO TÍTULO \\ $\mathrm{DE}$ \\ Mestre em CiênCIAS
}

Programa: Matemática
Orientador: Prof. Dr. Vyacheslav Futorny

Durante o desenvolvimento deste trabalho

o autor recebeu auxílio financeiro do CNPQ, processo 134228/2013-8.

São Paulo, março de 201 Ј 


\section{Representações da álgebra de Lie \\ de campos vetoriais sobre um toro $\mathrm{N}$-dimensional}

Esta versão da tese contém as correções e alterações sugeridas pela Comissão Jülgadora durante a defesa da versão original do trabalho, realizada em 30/03/2015. Uma cópia da versão original está disponível no

Instituto de Matemática e Estatística da Universidade de São Paulo.

Comissão Julgadora:

- Prof. Dr. Vyacheslav Futorny (orientador) - IME-USP

- Prof. Dr. Plamen Koshlukov - UNICAMP

- Prof. Dr. Renato Alessandro Martins - UNIFESP 


\section{Resumo}

Zaidan, A. E. Representações da álgebra de Lie de campos vetoriais sobre um toro N-dimensional. 2015. 39 f. Dissertação (Mestrado) - Instituto de Matemática e Estatística, Universidade de São Paulo, São Paulo, 2015.

O objetivo deste texto é apresentar uma classe de módulos para álgebra de Lie de campos vetoriais em um toro $N$-dimensional, $\operatorname{Vect}\left(\mathbb{T}^{N}\right)$. O caso $N=1$ nos dá a famosa álgebra de Witt (sua extensão central é álgebra de Virasoro). A álgebra Vect $\left(\mathbb{T}^{N}\right)$ apresenta uma classe de módulos parametrizada por módulos de dimensão finita da álgebra $\mathfrak{g l}_{N}$.

Nosso objeto central de estudo são módulos induzidos dos módulos tensoriais $\operatorname{de~} \operatorname{Vect}\left(\mathbb{T}^{N}\right)$ para $\operatorname{Vect}\left(\mathbb{T}^{N+1}\right)$. Estes módulos apresentam um quociente irredutível com espaços de peso de dimensão finita.

A álgebra Vect $\left(\mathbb{T}^{N}\right)$ apresenta como subálgebra $\widetilde{\mathfrak{s l}}_{N+1}$. Com a restrição da ação $\operatorname{de} \operatorname{Vect}\left(\mathbb{T}^{N}\right)$ a esta subálgebra obtemos o carácter deste quociente.

Para obter um critério de irredutibilidade e construir sua realização de campo livre, consideramos uma classe de módulos para $\Omega^{1}\left(\mathbb{T}^{N+1}\right) / \mathrm{d} \Omega^{0}\left(\mathbb{T}^{N+1}\right) \rtimes \operatorname{Vect}\left(\mathbb{T}^{N}\right)$, construída a partir de álgebras de vértice. Quando restritos a Vect $\left(\mathbb{T}^{N}\right)$ estes módulos continuam irredutíveis a menos que apareçam no chiral de de Rham.

Palavras-chave: Álgebra Toroidal Completa, Álgebra de Vértice, Álgebra de Witt, Chiral de de Rham, Módulos Tensoriais. 


\section{Abstract}

Zaidan, A. E.Representation of the Lie Algebra of Vector Fields on a $N$-Dimensional Torus. 2015. 39 f. Dissertação (Mestrado) - Instituto de Matemática e Estatística, Universidade de São Paulo, São Paulo, 2015.

The goal of this text is to present a class of modules for the Lie algebra of vector fields in a $N$-dimensional torus, $\operatorname{Vect}\left(\mathbb{T}^{N}\right)$. The case $N=1$ give us the famous Witt algebra (its central extension is the Virasoro algebra). The algebra $\operatorname{Vect}\left(\mathbb{T}^{N}\right)$ has a class of modules parametrized by finite dimensional $\mathfrak{g l}_{N}$-modules.

The central object of our study are modules induced from tensor modules for $\operatorname{Vect}\left(\mathbb{T}^{N}\right)$ to $\operatorname{Vect}\left(\mathbb{T}^{N+1}\right)$. Those modules have an irreducible quotient such that every weight space has finite dimension.

The algebra $\operatorname{Vect}\left(\mathbb{T}^{N}\right)$ has as subalgebra $\widetilde{\mathfrak{s l}}_{N+1}$. Restricting the action of $\operatorname{Vect}\left(\mathbb{T}^{N}\right)$ to this subalgebra we have the character of this quotient.

To obtain a critreria for irreducibility and construct a free field reazilation, we consider a class of modules for $\Omega^{1}\left(\mathbb{T}^{N+1}\right) / \mathrm{d} \Omega^{0}\left(\mathbb{T}^{N+1}\right) \rtimes \operatorname{Vect}\left(\mathbb{T}^{N}\right)$, constructed from vertex algebras. When restricted to $\operatorname{Vect}\left(\mathbb{T}^{N}\right)$ thesse modules remain irreducible, unless they belongs to the chiral de Rham complex. Keywords: Chiral de Rham Complex, Full Toroidal Lie Algebra, Tensor Modules, Vertex Algebra, Witt Algebra. 


\section{Sumário}

Lista de Símbolos $\quad$ vii

Introdução

1 Módulos de Peso e Carácter 3

1.1 Módulos de peso . . . . . . . . . . . . . . . . . . . . 3

1.2 Módulos de Verma . . . . . . . . . . . . . . . . . . . . . 4

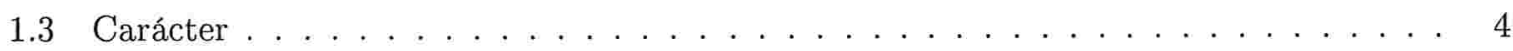

2 Álgebra $\operatorname{Vect}\left(\mathbb{T}^{N}\right) \quad 7$

2.1 Álgebra de Witt . . . . . . . . . . . . . . . . . . 7

2.2 Extensões Centrais e Generalização da Álgebra de Witt . . . . . . . . . . . . . . . . . 8

2.3 Álgebra Toroidal . . . . . . . . . . . . . . . . . . . . . 8

3 Módulo Tensorial $\quad 11$

3.1 Módulo Tensorial . . . . . . . . . . . . . . . . . . . . . . . . 11

3.2 Complexo de de Rham . . . . . . . . . . . . . . . . . 12

3.3 Um Teorema de Rao . . . . . . . . . . . . . . . . . . . . . . . . . 12

3.4 Carácteres dos Submódulos . . . . . . . . . . . . . . . . . . . 14

4 Superálgebra de Vértice $\quad 15$

4.1 Definições e Notações . . . . . . . . . . . . . . . . . . . . . . . 15

4.2 Superálgebras de Lie de Vértice . . . . . . . . . . . . . . . . . 16

5 Módulos Limitados $\quad 21$

5.1 Módulos Induzidos . . . . . . . . . . . . . . . . . . . 21

5.2 Carácter dos Módulos Induzidos . . . . . . . . . . . . . . . . . 23

5.3 Critério para Irredutibilidade . . . . . . . . . . . . . . . . . . . . 24

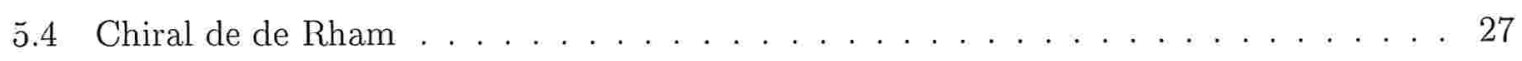

A Âlgebras de Lie $\quad 31$

$\begin{array}{ll}\text { B Representações } & 37\end{array}$

Referências Bibliográficas $\quad 39$ 
vi SUMARIO 


\section{Lista de Símbolos}

$\begin{array}{ll}V_{\lambda} & 3 \\ M(\lambda) & 4 \\ L(\lambda) & 4 \\ M(V) & 4 \\ \chi(V) & 5 \\ t^{r} & 8 \\ d_{a} & 8 \\ \mathfrak{h}_{\text {Vect }\left(\mathbb{T}^{n}\right)} & 8 \\ T(W, \gamma) & 11 \\ q^{\gamma} \Omega^{k}\left(\mathbb{T}^{n}\right) & 12 \\ W(\gamma, k) & 12 \\ \widehat{W}(\gamma, k) & 12 \\ M_{*} & 12 \\ Y(\cdot, z) & 15 \\ a(z)- & 16 \\ : a(z) b(z): & 16 \\ V_{\text {Hyp }}^{+} & 17 \\ V_{\mathfrak{g l}_{n}} & 18 \\ V_{\mathbb{Z}^{N}} & 19 \\ \mathcal{D} & 21 \\ M(T) & 21 \\ L(W, \gamma, h) & 23 \\ V_{\mathbb{Z}^{N}}^{k} & 28 \\ Q & 28 \\ \mathrm{~d} & 28\end{array}$




\section{Introdução}

Recentemente, uma nova área da teoria das representações surgiu, a teoria de módulos limitados para álgebra de Lie de dimensão infinita com uma $\mathbb{Z}^{N}$-graduação densa. Para $N=1$ temos, como exemplo, as álgebras de Kac-Moody afim e a álgebra de Virasoro. Neste caso, a principal ferramenta para a teoria das representação é o conceito de módulo de Verma. No entanto, para $N>1$ não há maneira natural de se dividir $\mathbb{Z}^{N} \backslash\{0\}$ em partes positiva e negativa.

Considere a álgebra de polinômios de Laurent em $N$ variáveis complexas, $\mathbb{C}\left[t_{1}^{ \pm 1}, \ldots, t_{N}^{ \pm 1}\right]$. Sua álgebra de derivações, chamada de álgebra de Witt, que é isomorfa a álgebra de campos vetoriais em um toro $\mathrm{N}$-dimensional, Vect $\left(\mathbb{T}^{N}\right)$ é um exemplo de uma álgebra com uma $\mathbb{Z}^{N}$-graduação densa. Esta álgebra apresenta uma classe de módulos de origem geométrica, que decorre dos elementos da álgebra agirem em campos tensoriais como derivadas de Lie. Estes módulos, chamados de módulos tensoriais, são parametrizados a partir de módulos de $\mathfrak{g l}_{N}$ de dimensão finita.

Um teorema de $\mathrm{E}$. Rao garante que módulos irredutíveis de $\mathfrak{g l}_{N}$ dão origem a módulos tensoriais irredutíveis para Vect $\left(\mathbb{T}^{N}\right)$, salvo quando estes pertencerem ao complexo de de Rham, os módulos de $k$-formas diferenciais. Mesmo estes sendo irredutíveis, seus módulos tensoriais podem não ser irredutíveis.

Para dividir esta álgebra em parte positiva e negativa consideramos uma coordenada especial, no nosso caso tomamos a coordenada $t_{0}$ da álgebra $\mathcal{D}=\operatorname{Vect}\left(\mathbb{T}^{N+1}\right)$. Para a decomposição triangular consideramos a $\mathbb{Z}$-graduação induzida pelo expoente da coordenada $t_{0}$. Podemos expandir a ação de $\operatorname{Vect}\left(\mathbb{T}^{N}\right)$ no módulo tensorial para a parte positiva e nula da decomposição triangular. Assim, podemos induzir a ação para toda álgebra, gerando uma nova classe de módulos

$$
M(T)=\operatorname{Ind}_{\mathcal{D}_{0} \oplus \mathcal{D}_{+}}^{\mathcal{D}} T .
$$

Estes módulos têm seus espaços de peso com dimensão infinita, porém, um teorema de S. Berman e Y. Billig [1] garantem que o quociente

$$
L(T)=M(T) / M^{r a d},
$$

onde $M^{\text {rad }}$ é o submódulo maximal, tem os espaços de peso com dimensão finita.

Temos então dois problemas com relação a estes módulos, determinar seu carácter e obter um critério de irredutibilidade. $\mathrm{O}$ caso $N=1$ foi estudado por Y. Billig, A Molev e R. Zhang [4]. No entanto o médoto aplicado por eles não funcionou para $N$ maiores.

Uma álgebra toroidal completa é o produto semidireto da extensão central da álgebra multiloop de uma álgebra de Lie pela álgebra de campos vetoriais em um toro. Um outro artigo de Y. Billig [2] apresenta uma categoria de módulos para álgebra toroidal completa utilizando álgebras de vértice. A partir desse artigo obtemos uma representação de campo livre para estes módulos, e a partir dela respondemos as duas perguntas.

Para o carácter devemos considerar a ação da subálgebra $\tilde{\mathfrak{s l}}_{N+1} \subset \operatorname{Vect}\left(\mathbb{T}^{N+1}\right)$. Obtemos que o carácter coincide com um módulo de Verma generalizado para $\mathfrak{s l}_{N+1}$, porém o módulo não é isomorfo a um módulo de Verma generalizado. Estes módulos foram chamados de módulos de Wakimoto generalizados, por Y. Billig e V. Futorny [3].

Para o problema de irredutibilidade um teorema de Y. Billig e V. Futorny [3] garante condições para certos módulos serem irredutíveis. Os módulos que este teorema não garante irredutibilidade 
aparecem em uma estrutura algébrica chamada Chiral de de Rham. A partir do operador diferencial deste Chiral temos que estes módulos são na verdade redutíveis.

\section{Organização do Trabalho}

No capítulo 1 apresentamos as definições de módulo de peso e de Verma, também apresentamos a definição e propriedades do carácter de um módulo. Estas definições serão amplamente utilizadas no texto.

No capítulo 2 apresentamos nosso objeto de estudo, a álgebra de campos vetoriais em um toro $N$-dimensional, Vect $\left(\mathbb{T}^{N}\right)$. Apresentamos uma motivação física, a álgebra de Virasoro, que é uma extensão central daquela álgebra. Por fim apresentamos a álgebra toroidal completa.

No capítulo 3 apresentamos um módulo para a álgebra Vect $\left(\mathbb{T}^{n}\right)$, o módulo tensorial $T(W, \gamma)$. Apresentamos um teorema que descreve quando os módulos tensoriais são irredutíveis. Nos casos em que o módulo tensorial não é irredutível, um teorema de Eswara Rao fornece muita informação sobre as componentes irredutíveis. Uma novidade que aparece neste texto são os seus caracteres que aparecem no final do capítulo.

No capítulo 4 é apresentada a estrutura de superálgebra de vértice e algumas de suas propriedades. Quatro dessas superálgebras são apresentadas, estas desempenham papel chave na representação de campos livres de $\operatorname{Vect}\left(\mathbb{T}^{N}\right)$.

Finalmente, no último capítulo são apresentados dois teoremas de Vyacheslav Futorny e Yuly Billig, o primeiro apresenta o carácter de módulos induzidos de $\operatorname{Vect}\left(\mathbb{T}^{N}\right)$ e o segundo expõe quais condições estes módulos devem satisfazer para serem irredutíveis. Por fim, apresentamos a estrutura do Chiral de de Rham.

Para compreensão do texto é recomendável uma certa familiaridade com álgebras de Lie e representações de estruturas algébricas. Não possuindo-a não se desencoraje, os apêndices apresentam toda informação necessária para acompanhar o texto para quem fez um curso básico de álgebra linear e saiba as propriedades básicas do produto tensorial. 


\section{Capítulo 1}

\section{Módulos de Peso e Carácter}

Neste capítulo são apresentadas as definições e algumas propriedades de módulo de peso e de caráter de módulo, seguindo [9], e também apresentamos as fórmulas dos caracteres dos módulos de Verma $U\left(\tilde{\mathfrak{s l}}_{N}^{-}\right)$e $U\left(\widehat{\mathfrak{g l}}_{N}^{-}\right)$que serão utilizadas mais adiante no texto.

\subsection{Módulos de peso}

Seja g uma álgebra de Lie semi-simples de dimensão finita sobre um corpo $k$ de característica $0, \mathfrak{h}$ uma subálgebra de Cartan de $\mathfrak{g}, \Phi$ um sistema de raízes e $V$ um $\mathfrak{g}$-módulo de dimensão finita. Sabemos que se $V$ for de dimensão finita, então todo elemento de $\mathfrak{h}$ será diagonalizável. Como $\mathfrak{h}$ é abeliana, temos que os elementos de $\mathfrak{h}$ são simultaneamente diagonalizáveis. Assim, para todo $\lambda \in \mathfrak{h}^{*}$, podemos definir $V_{\lambda}:=\{v \in V \mid \forall h \in \mathfrak{h}, h . v=\lambda(h) v\}$ e teremos

$$
V=\bigoplus_{\lambda \in \mathfrak{h}^{*}} V_{\lambda}
$$

Quando $V_{\lambda} \neq 0$ dizemos que $V_{\lambda}$ é um espaço de peso e que $\lambda$ é um peso de $V$. Mesmo no caso de dimensão infinita, se $V$ for soma de espaços de peso, diremos que $V$ é um módulo de peso. Aqui vale observar que os $\lambda$ podem ser vistos como auto-valores generalizados.

Exemplo 1. A álgebra g pode agir sobre si mesma pela representação adjunta, neste caso os pesos são as raízes de $\mathfrak{g}$.

Para $\Phi$, seja $\Phi^{+}$uma escolha de raízes positivas, dizemos que (para esta escolha de raízes positivas) $v \neq 0$ é um vetor de peso máximo se para todo $\alpha \in \Pi^{+}, \mathfrak{g}_{\alpha} v=0$, onde $\mathfrak{g}_{\alpha}$ é o espaço de peso $\alpha$ da representação adjunta. No caso de um módulo ser gerado por um vetor de peso máximo, diremos que este módulo é de peso máximo. Além disso, podemos definir $\mathfrak{g}^{+}=\oplus_{\alpha \in \Pi^{+}} \mathfrak{g}_{\alpha} \mathrm{e}$ $\mathfrak{g}^{-}=\oplus_{\alpha \in \Pi^{-}} \mathfrak{g}_{\alpha}$ onde $\Phi^{-}=-\Phi^{+}$, e temos a seguinte decomposição $\mathfrak{g}=\mathfrak{g}^{-} \oplus \mathfrak{h} \oplus \mathfrak{g}^{+}$, que chamaremos de decomposição triangular de $\mathfrak{g}$.

Exemplo 2. Seja $\mathfrak{g}=\mathfrak{s l}_{2}$ e $k=\mathbb{C}$, e tome a seguinte base para $\mathfrak{g}$

$$
f=\left(\begin{array}{cc}
0 & 0 \\
1 & 0
\end{array}\right), \quad h=\left(\begin{array}{cc}
1 & 0 \\
0 & -1
\end{array}\right), \quad e=\left(\begin{array}{ll}
0 & 1 \\
0 & 0
\end{array}\right) .
$$

Tomemos a seguinte subálgebra de Cartan $\mathfrak{h}=\langle h\rangle=\mathbb{C} h$ e como parte positiva $\mathfrak{g}^{+}=\langle e\rangle=\mathbb{C} e$, e seja $V:=M(\lambda)$ o módulo gerado pelo vetor $v$ de peso máximo $\lambda$. Vamos mostrar como $e$ e $f$ agem neste módulo. Temos que $e . v=0$ já que $v$ é de peso máximo, para $f$ temos

$$
h . f . v=f . h . v+[h, f] . v=\lambda f . v-2 f . v=(\lambda-2) f . v .
$$

Mais geralmente, seja $v_{0}=v$ e $v_{i}=f \cdot v_{i-1}$ temos

$$
h . f \cdot v_{i}=f \cdot h \cdot v_{i}+[h, f] \cdot v_{i}=(\lambda-2 i) f \cdot v_{i}-2 f \cdot v_{i}=(\lambda-2(i+1)) f \cdot v_{i} .
$$


Quanto à ação de $e$ temos

$$
e . v_{1}=e . f . v_{0}=f . e . v_{0}+[e, f] v_{0}=h . v_{0}=\lambda v_{0} .
$$

Para $i>0$, temos

$e . v_{i}=e . f . v_{i-1}=f . e . v_{i-1}+h v_{i-1}=f . e . v_{i-1}+(\lambda-2(i-1)) v_{i-1}=\sum_{n=0}^{i-1}(\lambda-2 n) v_{i-1}=i(\lambda+1-i) v_{i-1}$.

Em resumo, temos o seguinte diagrama, onde cada ponto é um espaço de peso.

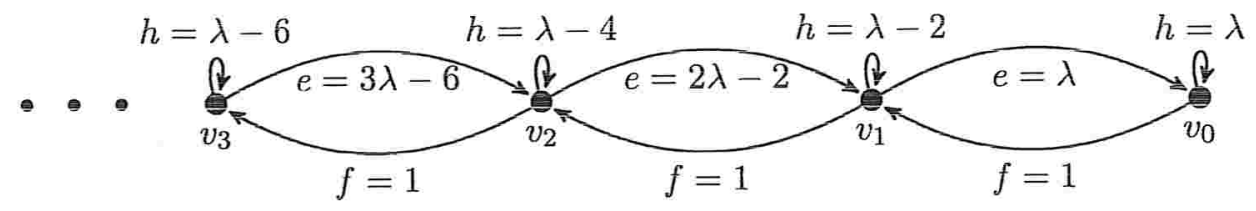

Podemos observar que se, e somente se, $\lambda$ for inteiro positivo, existirá $i$ tal que $i(\lambda+1-i)=0$, mais precisamente $i=\lambda+1$, ou seja, o espaço de peso $V_{-\lambda-2}\left(=\mathbb{C} v_{\lambda+1}\right)$ será anulado por $e$, disso segue que

$$
L:=\bigoplus_{n=-\lambda-1}^{-\infty} \mathbb{C} v_{n}
$$

é um submódulo de $V$. Este submódulo é o submódulo maximal de $M(\lambda)$ e assim definimos $L(\lambda):=$ $M(\lambda) / L$. Com isso, temos todos os módulos de dimensão finita de $\mathfrak{s t}_{2}$. Com efeito, dado um módulo de dimensão finita $n$, este será isomorfo a $L(n-1)$.

\subsection{Módulos de Verma}

Do último exemplo de seção anterior podemos ver que o módulo $M(\lambda)$ poderia ser definido como $U\left(\mathfrak{g}^{-}\right) v_{0}=\left\{y \cdot v_{0} \mid y \in U\left(\mathfrak{g}^{-}\right)\right\}$, onde $U\left(\mathfrak{g}^{-}\right)$é a álgebra universal envelopante de $\mathfrak{g}^{-}$. Isto nos leva a definição de módulo de Verma, nomeado em homenagem ao matemático Daya-Nand Verma.

Definição 1.2.1. Seja $\lambda \in \mathfrak{h}^{*}$, e seja $k_{\lambda}$ um módulo unidimensional para a sub-álgebra de Borel $\mathfrak{b}:=\mathfrak{b} \oplus \mathfrak{g}^{+}$, onde $h \in \mathfrak{h}$ age por multiplicação por $\lambda(h)$ e $\mathfrak{g}^{+}$age trivialmente. Definimos o módulo de Verma de $\mathfrak{g}$ de peso máximo $\lambda$ como sendo $M(\lambda)=U(\mathfrak{g}) \otimes_{U(\mathfrak{b})} k_{\lambda}$. Além disso, definimos $L(\lambda)=M(\lambda) / L$, onde $L$ é o submódulo maximal de $M(\lambda)$.

O Teorema PBW (veja B.0.11) implica que $M(\lambda)$ é isomorfo a $U\left(\mathfrak{g}^{-}\right) \otimes k_{\lambda}$.

Uma generalização que podemos fazer com os módulos de Verma é no lugar de fazermos o produto tensorial por módulo unidimensional $F_{\lambda}$ de $\mathfrak{b}$, podemos fazê-lo por um módulo qualquer (de dimensão finita) $V$ de uma sub-álgebra parabólica p, i. e., uma sub-álgebra que contém uma sub-álgebra de Borel.

Definição 1.2.2. Seja $V$ um módulo de dimensão finita para a sub-álgebra parabólica p. Definimos $M(V):=U(\mathfrak{g}) \otimes_{U(\mathfrak{b})} V$ como sendo o módulo de Verma generalizado.

Novamente por PBW temos $M(V) \simeq U\left(\mathfrak{g}^{-}\right) \otimes V$. A partir de agora iremos nos referir ao módulo de Verma generalizado pela sua sigla em inglês, GVM.

\subsection{Carácter}

Dado um módulo de peso $V$, uma outra maneira de descrevê-lo é através da dimensão dos seus espaços de pesos. Em $\mathbb{Z}\left[\left[\mathfrak{h}^{*}\right]\right]$, definimos, para cada $\lambda \in \mathfrak{h}^{*}$, o símbolo formal $e^{\lambda}$. Estes símbolos formais definem uma base de $\mathbb{Z}\left[\left[\mathfrak{h}^{*}\right]\right]$. Com isto estamos prontos para apresentar a definição de caráter de um módulo. 
Definição 1.3.1. Dado um módulo de peso $V$, a seguinte soma formal $\chi(V) \in \mathbb{Z}\left[\left[\mathfrak{h}^{*}\right]\right]$ é definida como sendo o carácter do módulo $V$.

$$
\chi(V):=\sum_{\lambda \in \mathfrak{h}^{*}} \operatorname{dim} V_{\lambda} e^{\lambda}
$$

Exemplo 3. Seja $\mathfrak{g}=\mathfrak{s l}_{2}$ e considere $V=L(2)$. Temos que $V=V_{-2} \oplus V_{0} \oplus V_{2}$, cada um destes espaços de peso tem dimensão um. Assim, o caráter de $V$ é dado por

$$
\chi(V)=e^{-2}+e^{0}+e^{2} .
$$

Agora, se considerarmos $V=M(\lambda)$, para $\lambda$ qualquer, temos

$$
\chi(V)=\sum_{i \in \mathbb{Z}^{+}} e^{\lambda-2 i}=\frac{e^{\lambda}}{1-e^{-2}} .
$$

Proposição 1.3.2. Sejam g uma álgebra de Lie e $V$ e $W$ dois g-módulos, então temos que

$$
\chi(V \oplus W)=\chi(V)+\chi(W) \quad e \quad \chi(V \otimes W)=\chi(V) \chi(W) .
$$

Geralmente, o carácter de um módulo de Verma é representado pelo carácter da envelopante universal da parte negativa. Em particular, o caracteres dos módulos de Verma das álgebras $\widetilde{\mathfrak{s l}}_{N}=$ $\mathbb{C}\left[t^{ \pm 1}\right] \otimes \mathfrak{s l}_{N}$ e $\widehat{\mathfrak{g l}}_{N}=\mathbb{C}\left[t^{ \pm 1}\right] \otimes \mathfrak{g l}_{N} \oplus \mathbb{C} C$ são apresentado pela seguinte proposição:

Proposição 1.3.3. Temos que

$$
\chi\left(U\left(\tilde{\mathfrak{s l}}_{N}^{-}\right)\right)=\prod_{k=1}^{\infty}\left(1-s^{k}\right)^{-N^{2}+1} \text { e } \chi\left(U\left(\widehat{\mathfrak{g l}}_{N}^{-}\right)\right)=\prod_{k=1}^{\infty}\left(1-s^{k}\right)^{-N^{2}} .
$$

Demonstração. Obter estas fórmulas elegantes para os caracteres exige um conhecimento de fórmulas de caracteres, uma boa fonte que tem essas fórmulas é [12]. Aqui iremos mostrar como calcular a dimensão de um dado peso para um modulo de Verma para a álgebra $\tilde{\mathfrak{s l}}_{N}^{-}$(Para ver as estruturas das raízes veja o Apêndice A).

Por exemplo, tome $\mu=\lambda-3 \alpha_{1}-2 \alpha_{2}$ Vamos calcular a dimensão de $V_{\mu}$ em $M(\lambda)$. Témos as seguintes maneiras de chegarmos a $\mu$ partindo de $\lambda$ utilizando as raízes:

$$
\begin{aligned}
& \text { - }-\alpha_{1}-\alpha_{1}-\alpha_{1}-\alpha_{2}-\alpha_{2} \\
& \text { - }-\alpha_{1}-\alpha_{1}-\left(\alpha_{1}+\alpha_{2}\right)-\alpha_{2} \\
& \text { - }-\alpha_{1}-\left(\alpha_{1}+\alpha_{2}\right)-\left(\alpha_{1}+\alpha_{2}\right) \\
& \text { - }-\alpha_{1}-\left(2 \alpha_{1}+\alpha_{2}\right)-\alpha_{2} \\
& \text { - }-\left(2 \alpha_{1}+\alpha_{2}\right)-\left(\alpha_{1}+\alpha_{2}\right) \\
& \text { - }-\alpha_{1}-\alpha_{1}-\left(\alpha_{1}+2 \alpha_{2}\right) \\
& \text { - }-\left(3 \alpha_{1}+2 \alpha_{2}\right)
\end{aligned}
$$

Logo, $\operatorname{dim}\left(V_{\mu}\right)=7$. 


\section{Capítulo 2}

\section{Álgebra $\operatorname{Vect}\left(\mathbb{T}^{N}\right)$}

Neste capítulo apresentamos inicialmente a álgebra de Witt, que coincide com a álgebra de campos vetoriais em um círculo. Em seguida apresentamos a definição de extensão central e como exemplo a álgebra de Virasoro, extensão central da álgebra de Witt. Aqui apresentamos nosso objeto de estudo, uma generalização da álgebra de Witt, a álgebra de campos vetoriais em um toro, $\operatorname{Vect}\left(\mathbb{T}^{N}\right)$. Por fim, apresentamos uma álgebra que será importante mais adiante, a álgebra toroidal completa.

\section{1 Âlgebra de Witt}

A álgebra de Witt $W$, nomeada em homenagem ao matemático Ernest Witt, é a álgebra de derivações do anel $\mathbb{C}\left[z^{ \pm 1}\right]$. Definimos, para $n, m \in \mathbb{Z}$

$$
L_{n}:=-z^{n+1} \frac{\partial}{\partial z} \quad \text { e } \quad\left[L_{m}, L_{n}\right]:=(m-n) L_{m+n}
$$

No apêndice A mostramos que os $L_{n}$ formam uma base de $W$.

Uma outra maneira de enxergar esta álgebra é pelo seu carácter geométrico. Usualmente o conjunto de campo de vetores de uma variedade $M$ é denotado por $\mathfrak{X}(M)$, no entanto, estamos interessados nos campos que sejam analíticos, i. e., campos em que suas coordenadas sejam funções analíticas. Este conjunto de campos analíticos tem uma estrutura natural de álgebra de Lie, que denotaremos por Vect $(M)$. Esta estrutura decorre do fato de campos vetoriais serem uma derivada, mais explicitamente, sejam $X, Y \in \operatorname{Vect}(M)$ e $h: M \rightarrow k$ analítica, definimos

$$
[X, Y](h)=X(Y(h))-Y(X(h)) .
$$

Neste caso, como era para ser, o colchete é bilinear para escalares mas não para funções. Para ver como uma função pode ser retirada do colchete, devemos lembrar da regra de Leibniz da derivada do produto. Sejam $f, g: M \rightarrow k$ analíticas, temos que

$$
\begin{gathered}
{[f X, g Y](h)=f X(g Y(h))-g Y(f X(h))=f X(g) Y(h)+f g X(Y(h)-g Y(f) X(h)-g f Y(X(h)) \Rightarrow} \\
\Rightarrow[f X, g Y]=f g[X, Y]+f X(g) Y-g Y(f) X .
\end{gathered}
$$

Para $M=\mathbb{S}^{1}$, o círculo unitário, considere a coordenada $\theta$. Definimos

$$
L_{n}:=-\theta^{n+1} \frac{\partial}{\partial \theta}
$$

e é fácil ver que os $L_{n}$ formam uma base para $\operatorname{Vect}\left(\mathbb{S}^{1}\right)$. Seu colchete é dado por

$$
\left[L_{m}, L_{n}\right]=\left[\theta^{m+1} \frac{\partial}{\partial \theta}, \theta^{n+1} \frac{\partial}{\partial \theta}\right]=\theta^{m+n+2}\left[\frac{\partial}{\partial \theta}, \frac{\partial}{\partial \theta}\right]+(n+1) \theta^{m+n+1} \frac{\partial}{\partial \theta}-(m+1) \theta^{m+n+1} \frac{\partial}{\partial \theta}=
$$




$$
=(m-n) L_{m+n}
$$

\subsection{Extensões Centrais e Generalização da Âlgebra de Witt}

Definição 2.2.1. Diremos que $\dot{\mathfrak{g}}$ é uma extensão central de $\mathfrak{g}$ se existir uma sequencia exata curta

$$
0 \longrightarrow \mathfrak{z} \longrightarrow \dot{\mathfrak{g}} \longrightarrow \mathfrak{g} \longrightarrow 0
$$

com a imagem de $\mathfrak{z}$ coincidindo com o centro de $\dot{\mathfrak{g}}$.

A álgebra de Virasoro é uma extensão central da álgebra de Witt. Ela é gerada por $\left\{L_{n}, n \in\right.$ $\mathbb{Z}\} \cup\{c\}$ e as seguintes relações

$$
\left[L_{m}, L_{n}\right]=(m-n) L_{m+n}+\frac{c}{12}\left(m^{3}-m\right) \delta_{m+n, 0},\left[L_{n}, c\right]=0 .
$$

Apesar de ser primeiro descrita como extensão central da álgebra de Witt, a álgebra de Virasoro tem seu nome em homenagem ao físico Miguel Ângel Virasoro, pois este apresentou operadores do seu estudo de modelos de ressonâncias duais. Está álgebra ainda hoje tem mostrado várias aplicações na física.

Uma generalização natural de álgebra de Witt é considerar o anel $A_{N}:=\mathbb{C}\left[t_{1}^{ \pm 1}, t_{2}^{ \pm 1}, \ldots, t_{N}^{ \pm 1}\right]$. Definimos como a álgebra de Witt, $W^{N}$, a álgebra de derivação de $A_{N}$, simbolicamente, $W^{N}:=$ $\operatorname{Der} A^{N}$. Seja $\mathbb{T}^{N}=\mathbb{S}^{1} \times \ldots \times \mathbb{S}^{1}$, o toro $\mathbb{N}$-dimensional, podemos considerar $W^{N} \simeq \operatorname{Vect}\left(\mathbb{T}^{N}\right)$. De fato, pelo teorema de Schwarz temos que o colchete $\left[\partial / \partial t_{i}, \partial / \partial t_{j}\right]=0$ para $i, j \in\{1, \ldots, N\}$.

Uma notação que será muito útil é a notação de multi-índice, que é definida da seguinte forma, dado $r \in \mathbb{Z}^{n}$ então $t^{r}:=t_{1}^{r_{1}} t_{2}^{r_{2}} \ldots t_{n}^{r_{n}}$. Além disso, definimos $d_{a}:=t_{a} \partial / \partial t_{a}$ e é fácil ver que $\left\{t^{r} d_{a} \mid r \in \mathbb{Z}^{n}, a \in\{1, \ldots, n\}\right\}$ é uma base $\operatorname{de} \operatorname{Vect}\left(\mathbb{T}^{N}\right)$.

Com isso, o colchete em Vect $\left(\mathbb{T}^{N}\right)$ é dado por, sejam $a, b \in\{1, \ldots, N\}$ e $r, m \in \mathbb{Z}^{N}$,

$$
\left[t^{r} d_{a}, t^{m} d_{b}\right]=t^{r+m}\left[d_{a}, d_{b}\right]+m_{b} t^{r+m} d_{b}-r_{a} t^{r+m} d_{a}=m_{b} t^{r+m} d_{b}-r_{a} t^{r+m} d_{a} .
$$

$\operatorname{Em} \operatorname{Vect}\left(\mathbb{T}^{N}\right)$ definimos a seguinte $\operatorname{CSA}, \mathfrak{h}_{\operatorname{Vect}\left(\mathbb{T}^{N}\right)}:=\left\langle d_{1}, \ldots, d_{N}\right\rangle$. Uma propriedade que será de grande importância é que Vect $\left(\mathbb{T}^{N}\right)$ contém uma cópia de $\mathfrak{s l}_{N+1}$. De fato, temos a seguinte inclusão para $i, j \in\{1, \ldots, N\}, E_{i j} \rightarrow t_{i} t_{j}^{-1} d_{j}, E_{i, N+1} \rightarrow-t_{i} D, E_{N+1, j} \rightarrow t_{j}^{-1} d_{j}$ e $E_{N+1, N+1} \rightarrow-D$, onde $D:=\sum_{k=1}^{N} d_{k}$ e $E^{i j}:=\left(\delta_{i k} \delta_{j l}\right)_{k, l} \in \mathfrak{g l}_{N}$. Além disso, se considerarmos $\operatorname{Vect}\left(\mathbb{T}^{n+1}\right):=$ $\oplus_{p=0}^{n} \mathbb{C}\left[t_{0}^{ \pm 1}, t_{1}^{ \pm 1}, \ldots, t_{N}^{ \pm 1}\right] d_{p}$, fica claro que $\widetilde{\mathfrak{s l}}_{N+1}:=\mathbb{C}\left[t_{0}^{ \pm 1}\right] \otimes \mathfrak{s l}_{N+1} \subset \operatorname{Vect}\left(\mathbb{T}^{N}\right)$. Este fato será importante mais adiante.

\section{3 Álgebra Toroidal}

Álgebras toroidais são, de uma maneira bem natural, generalizações de álgebras de Kac-Moody afim.

Sejam $\dot{\mathfrak{g}}$ uma álgebra de Lie simples sobre $\mathbb{C}$ e $N>1$ um inteiro. Consideremos a álgebra de Lie $\mathcal{R} \otimes \dot{\mathfrak{g}}$ de aplicações de um toro $N$ dimensional em $\dot{\mathfrak{g}}$, onde $\mathcal{R}=\mathbb{C}\left[t_{0}^{ \pm}, t_{1}^{\frac{1}{\ddagger}}, \ldots, t_{N}^{ \pm}\right]$é a álgebra de polinômios de Fourier sobre um toro. A extensão central universal desta álgebra é devida a Kassel, sua construção é dada a seguir.

Seja $\Omega_{\mathcal{R}}^{1}\left(\mathbb{T}^{N}\right)$ o espaço das 1 -formas diferenciais em um toro: $\Omega_{\mathcal{R}}^{1}\left(\mathbb{T}^{N}\right)=\mathbb{D}_{p=0}^{N} \mathcal{R} d t_{p}$. Podemos escolher a seguinte base para $\Omega_{\mathcal{R}}^{1}\left(\mathbb{T}^{N}\right)$, como um $\mathcal{R}$-módulo, $\left\{k_{a}=t_{a}^{-1} d t_{a} \mid a=0, \ldots, N\right\}$. A derivada exterior é uma aplicação do espaço $\mathcal{R}$ em $\Omega_{\mathcal{R}}^{1}\left(\mathbb{T}^{N}\right)$ definida como $\mathrm{d}(f)=\sum_{p=0}^{N} t_{p}\left(\partial f / \partial t_{p}\right) k_{p} . \mathrm{O}$ centro $\mathcal{K}$ da extensão universal central $\dot{\mathfrak{g}} \otimes \mathcal{R} \oplus \mathcal{K}$ é realizado como

$$
\mathcal{K}=\Omega_{\mathcal{R}}^{1}\left(\mathbb{T}^{N}\right) / \mathrm{d}(\mathcal{R})
$$


e o colchete de Lie é dado por

$$
\left[f_{1}(t) \otimes g_{1}, f_{2}(t) \otimes g_{2}\right]=f_{1} f_{2} \otimes\left[g_{1}, g_{2}\right]+\left(g_{1} \mid g_{2}\right) \overline{f_{2} d f_{1}},
$$

onde $g_{1}, g_{2} \in \dot{\mathfrak{g}}, f_{1}, f_{2} \in \mathcal{R},(\cdot \cdot)$ é a forma de Killing de $\dot{\mathfrak{g}}$ e a barra em $\overline{f_{2} d f_{1}}$ indica a imagem pela projeção canônica $\Omega_{\mathcal{R}}^{1}\left(\mathbb{T}^{N}\right) \rightarrow \mathcal{K}$, no que se segue omitiremos esta barra para representar elementos de $\mathcal{K}$.

A ação natural de $\mathcal{D}=\operatorname{Vect}\left(\mathbb{T}^{N}\right)$ em $\mathcal{R} \otimes \dot{\mathfrak{g}}$ é dada por

$$
\left[t^{r} d_{a}, t^{m} g\right]=m_{a} t^{r+m} g
$$

esta ação é unicamente estendida a $\mathcal{K}$

$$
\left[t^{r} d_{a}, t_{b}^{k}\right]=m_{a} t^{r+m} k_{b}+\delta_{a b} \sum_{p=0}^{N} r_{p} t^{r+m} k_{p} .
$$

A álgebra toroidal completa é o produto semidireto

$$
\mathfrak{g}=(\mathcal{R} \otimes \dot{\mathfrak{g}} \oplus \mathcal{K}) \rtimes \mathcal{D} .
$$

Em [2] foi classificada uma categoria de módulos para a álgebra toroidal completa, tal categoria admite o seguinte caso particular $\dot{\mathfrak{g}}=0$. Neste caso obtemos módulos para a álgebra $\mathcal{K} \rtimes \mathcal{D}$. A técnica que utilizaremos será restringir estes módulos a ação de $\mathcal{D}$, o que veremos a seguir é que a maior parte destes módulos permanece irredutível quando restritos a ação de $\mathcal{D}$. 


\section{Capítulo 3}

\section{Módulo Tensorial}

Neste capítulo apresentamos o módulo tensorial como definido em [3]. Toda álgebra de Lie será sobre o corpo $\mathbb{C}$.

\subsection{Módulo Tensorial}

O módulo tensorial é um módulo para a álgebra $\operatorname{Vect}\left(\mathbb{T}^{N}\right)$ e é definido da seguinte forma.

Definição 3.1.1. Sejam $W$ um $\mathfrak{g l}_{N}$-módulo de dimensão finita e $\gamma \in \mathbb{C}^{N}$, então o espaço vetorial

$$
T(W, \gamma):=q^{\gamma} \mathbb{C}\left[q_{1}^{ \pm 1}, \ldots, q_{N}^{ \pm 1}\right] \otimes W
$$

é um $\operatorname{Vect}\left(\mathbb{T}^{N}\right)$-módulo com a ação dada por

$$
t^{r} d_{a}\left(q^{\mu} \otimes w\right):=\mu_{a} q^{\mu+r} \otimes w+\sum_{p=1}^{N} r_{p} q^{\mu+r} \otimes E^{p a} w,
$$

onde $r \in \mathbb{Z}^{N}, \mu \in \gamma+\mathbb{Z}^{N}, w \in W, a \in\{1, \ldots, N\}$ e $E^{p a}=\left(\delta_{p i} \delta_{a j}\right)_{i, j} \in \mathfrak{g l}_{N}$.

Vamos verificar que, de fato, $T(W, \gamma)$ é um $\operatorname{Vect}\left(\mathbb{T}^{N}\right)$-módulo. Temos por um lado

$$
\begin{gathered}
{\left[t^{r} d_{a}, t^{s} d_{b}\right]\left(q^{\mu} \otimes w\right)=\left(s_{a} t^{r+s} d_{b}-r_{b} t^{r+s} d_{a}\right)\left(q^{\mu} \otimes w\right)=} \\
=\left(s_{a} \mu_{b}-r_{b} \mu_{a}\right) q^{\mu+r+s} \otimes w+\sum_{p=1}^{N}(r+s)_{p}\left(s_{a}-r_{b}\right) q^{\mu+r+s} \otimes\left(E^{p b}-E^{p a}\right) w
\end{gathered}
$$

Por outro lado

$$
\begin{gathered}
t^{r} d_{a}\left(t^{s} d_{b}\left(q^{\mu} \otimes w\right)\right)=t^{r} d_{a}\left(\mu_{b} q^{\mu+s} \otimes w+\sum_{p=1}^{N} s_{p} q^{\mu+s} \otimes E^{p b} w\right)= \\
=\mu_{b}(\mu+s)_{a} q^{\mu+r+s} \otimes w+\sum_{p=1}^{N} \mu_{b} r_{p} q^{\mu+r+s} \otimes E^{p a} w+ \\
+\sum_{p=1}^{N} s_{p}(\mu+s)_{a} q^{\mu+r+s} \otimes E^{p b} w+\sum_{p=1}^{N} s_{a} r_{p} q^{\mu+r+s} \otimes E^{p b} w .
\end{gathered}
$$

Observe que $\sum_{p} r_{p} E^{p a} \mu=\mu_{a} r$. Por simetria temos

$$
\begin{gathered}
t^{s} d_{b}\left(t^{r} d_{a}\left(q^{\mu} \otimes w\right)\right)=\mu_{a}(\mu+r)_{b} q^{\mu+r+s} \otimes w+\sum_{p=1}^{N} \mu_{a} s_{p} q^{\mu+r+s} \otimes E^{p b} w+ \\
+\sum_{p=1}^{N}\left((r+s)_{p} r_{b}+r_{p} \mu_{b}\right) q^{\mu+r+s} \otimes E^{p a} w .
\end{gathered}
$$

Assim

$$
t^{r} d_{a}\left(t^{s} d_{b}\left(q^{\mu} \otimes w\right)\right)-t^{s} d_{b}\left(t^{r} d_{a}\left(q^{\mu} \otimes w\right)\right)=\left[t^{r} d_{a}, t^{s} d_{b}\right]\left(q^{\mu} \otimes w\right)
$$

Portanto, $T(W, \gamma)$ é um Vect $\left(\mathbb{T}^{N}\right)$-módulo 


\subsection{Complexo de de Rham}

A álgebra exterior $\bigwedge^{k}\left(\mathbb{C}^{N}\right)$ é um famoso gl ${ }^{N}$-módulo com a ação dada por

$$
X\left(v_{1} \wedge \ldots \wedge v_{k}\right)=\sum_{i=1}^{k} v_{1} \wedge \ldots v_{i-1} \wedge X v_{i} \wedge v_{i+1} \ldots \wedge v_{k}
$$

onde $X \in \mathfrak{g l}^{N}$ e $v_{j} \in \mathbb{C}^{N}, \forall j \in\{1, \ldots, k\}$. Seu módulo tensorial pode ser identificado como formas diferenciais via os seguintes isomorfismos

$$
q^{\mu} \otimes e_{i_{1}} \wedge \ldots \wedge e_{i_{k}} \rightarrow t^{\mu} t_{i_{1}}^{-1} d t_{i_{1}} \wedge \ldots \wedge t_{i_{k}}^{-1} d t_{i_{k}}
$$

assim, chamaremos estes de módulos tensoriais do complexo de de Rham,

$$
q^{\gamma} \Omega^{k}\left(\mathbb{T}^{N}\right):=T\left(\bigwedge^{k}\left(\mathbb{C}^{N}\right), \gamma\right)
$$

para $k \in\{1, \ldots, N-1\}$, e para $k \in\{0, N\}$ definimos os módulos tensoriais com $W$ sendo os $\mathfrak{g l}_{N}$-módulos onde a identidade age multiplicando por $k$, e a subálgebra $\mathfrak{s l}_{N}$ age trivialmente. $\mathrm{O}$ interessante é que a derivada exterior das formas diferencias é um morfismo de $\operatorname{Vect}\left(\mathbb{T}^{N}\right)$-módulos e, como já sabemos, a imagem pela derivada exterior nem sempre é todo espaço seguinte do complexo. Isso já nos mostra que estes módulos tensoriais não são irredutíveis, porêm, um teorema presente em [16] mostra que estes são exatamente os únicos redutíveis.

Teorema 3.2.1. Seja $W$ um $\mathfrak{g l}_{N}$-módulo irredutivel de dimensão finita, e seja $\gamma \in \mathbb{C}^{N}$, então o módulo tensorial $T(W, \gamma)$ será redutivel se ele aparecer no complexo de de Rham

$$
q^{\gamma} \Omega^{0}\left(\mathbb{T}^{N}\right) \stackrel{d}{\rightarrow} q^{\gamma} \Omega^{1}\left(\mathbb{T}^{N}\right) \stackrel{d}{\rightarrow} \ldots \stackrel{d}{\rightarrow} q^{\gamma} \Omega^{N}\left(\mathbb{T}^{N}\right)
$$

a menos dos termos extremos $(k=0, N)$ quando $\gamma \notin \mathbb{Z}^{N}$, estes e todos os demais serão irredutíveis.

\subsection{Um Teorema de Rao}

O seguinte teorema, devido a Eswara Rao, apareceu em [16], no entanto, seguiremos [7], pois este apresenta uma prova mais simples deste teorema. Inicialmente observe que se $\gamma \in \mathbb{Z}^{N}$, então $T(W, \gamma)=T(W, 0)$. Com isso definimos os seguintes subespaços de $q^{\gamma} \Omega^{k}\left(\mathbb{T}^{N}\right)$ para $k \in\{1, \ldots, N-$ 1\}

$$
W(\gamma, k):=\bigoplus_{\mu \in \gamma+\mathbb{Z}^{N}} q^{\mu} \otimes\left(\mathbb{C}(\mu) \wedge \mathbb{C}^{N} \wedge \ldots \wedge \mathbb{C}^{N}\right)
$$

E quando $\gamma=0$ definimos

$$
\widehat{W}(0, k):=W(0, k) \oplus q^{0} \otimes\left(\mathbb{C}^{N} \wedge \ldots \wedge \mathbb{C}^{N}\right) .
$$

Para $\gamma \neq 0$ também denotaremos $\widehat{W}(\gamma, k)=W(\gamma, k)$, por conveniência.

Dado um submódulo $M$ de $T(W, \gamma)$, definimos $M_{\mu}:=\left\{v \in W \mid q^{\mu} \otimes v \in M\right\}$ e $M_{*}:=\cap_{\mu \in \gamma+\mathbb{Z}^{N}} M_{\mu}$. O seguinte lema será utilizado no teorema de Rao.

Lema 3.3.1. Se $M_{*} \neq\{0\}$, então $M=T(W, \gamma)$.

Demonstração. Suponha $M_{*} \neq\{0\}$ e seja $w \neq 0 \in M_{*}$, temos que para todo $a \in\{1, \ldots, N\}$ e $r \in \mathbb{Z}^{N}$,

$$
t^{r} d_{a} q^{\mu-r} \otimes w=(\mu-r)_{a} q^{\mu} \otimes w+\sum_{p=1}^{k} r_{p} q^{\mu} \otimes E^{p a} w \Rightarrow E^{i j} w \in M_{\mu},
$$


para todo $i, j \in\{1, \ldots, N\}$ e $\mu \in \gamma+\mathbb{Z}^{N}$. Com isto temos que $M_{*}$ é um $g l_{N}$-submódulo de $W$, porém $W$ é irredutível, logo $M_{*}=W$ e, portanto, $M=T(W, \gamma)$.

Teorema 3.3.2. Para $k \in\{1, \ldots, N-1\}$ temos que $W(\gamma, k)$ e $q^{\gamma} \Omega^{k}\left(\mathbb{T}^{N}\right) / \widehat{W}(\gamma, k)$ são $\operatorname{Vect}\left(\mathbb{T}^{N}\right)$ módulos irredutiveis.

Demonstração. Considere a seguinte aplicação

$$
\begin{gathered}
\iota_{k}: q^{\gamma} \Omega^{k}\left(\mathbb{T}^{N}\right) \rightarrow q^{\gamma} \Omega^{k+1}\left(\mathbb{T}^{N}\right) \\
q^{\mu} \otimes\left(v_{1} \wedge v_{2} \wedge \ldots \wedge v_{k}\right) \rightarrow q^{\mu} \otimes\left(\mu \wedge v_{1} \wedge v_{2} \wedge \ldots \wedge v_{k}\right) .
\end{gathered}
$$

Temos que $\iota_{k}$ é um morfismo de $\operatorname{Vect}\left(\mathbb{T}^{N}\right)$-módulos. Com efeito, seja $r \in \mathbb{Z}^{N}$ e $a \in\{1, \ldots, N\}$ temos

$$
\begin{gathered}
\iota_{k}\left(t^{r} d_{a}\left(q^{\mu} \otimes\left(v_{1} \wedge \ldots \wedge v_{k}\right)\right)=\mu_{a} q^{\mu+r} \otimes\left((\mu+r) \wedge v_{1} \wedge \ldots \wedge v_{k}\right)+\right. \\
+\sum_{p=1}^{N} \sum_{i=1}^{k} r_{p} q^{\mu+r} \otimes\left((\mu+r) \wedge v_{1} \wedge \ldots \wedge E^{p a}\left(v_{i}\right) \wedge \ldots \wedge v_{k}\right)= \\
=\mu_{a} q^{\mu+r} \otimes\left(\mu \wedge v_{1} \wedge \ldots \wedge v_{k}\right)+\sum_{p=1}^{N} r_{p} q^{\mu+r} \otimes\left(E^{p a} \mu \wedge v_{1} \wedge \ldots \wedge v_{k}\right)+ \\
+\sum_{p=1}^{N} \sum_{i=1}^{k} r_{p} q^{\mu+r} \otimes\left(\mu \wedge v_{1} \wedge \ldots \wedge E^{p a}\left(v_{i}\right) \wedge \ldots \wedge v_{k}\right)+ \\
+\sum_{p=1}^{N} \sum_{i=1}^{k} r_{p} q^{\mu+r} \otimes\left(r \wedge v_{1} \wedge \ldots \wedge E^{p a}\left(v_{i}\right) \wedge \ldots \wedge v_{k}\right)=t^{r} d_{a}\left(\iota_{k}\left(q^{\mu} \otimes\left(v_{1} \wedge \ldots \wedge v_{k}\right)\right)\right.
\end{gathered}
$$

pois para todo $i$

$$
\sum_{p=1}^{N} r_{p}\left(r \wedge v_{1} \wedge \ldots \wedge E^{p a}\left(v_{i}\right) \wedge \ldots \wedge v_{k}\right)=\left(r \wedge v_{1} \wedge \ldots \wedge r\left(v_{i}\right)_{a} \wedge \ldots \wedge v_{k}\right)=0
$$

onde $\left(v_{i}\right)_{a}$ é a $a$-ésima coordenada do vetor $v_{i}$. É fácil ver que

$$
\operatorname{ker}\left(\iota_{k}\right)=\widehat{W}(\gamma, k), \operatorname{im}\left(\iota_{k}\right)=W(\gamma, k+1),
$$

e pelo primeiro teorema do isomorfismo

$$
q^{\gamma} \Omega^{k}\left(\mathbb{T}^{N}\right) / \widehat{W}(\gamma, k) \simeq W(\gamma, k+1) .
$$

Observe que $W(\gamma, 1)=\oplus_{\mu \in \gamma+\mathbb{Z}^{N}} \mathbb{C}\left(q^{\mu} \otimes \mu\right)$ e $t^{r} d_{a}\left(q^{\mu} \otimes \mu\right)=\mu_{a}\left(q^{\mu+r} \otimes(\mu+r)\right)$, para todo $a \in\{1, \ldots, N\}$ e $r \in \mathbb{Z}^{N}$, logo $W(\gamma, 1)$ é irredutível. Além disso $\widehat{W}(\gamma, N)=q^{\gamma} \Omega^{N}\left(\mathbb{T}^{N}\right)$. Dessa forma, para provar o teorema só precisamos provar que $q^{\gamma} \Omega^{k}\left(\mathbb{T}^{N}\right) / \widehat{W}(\gamma, k)$ são irredutíveis para $k \in\{1, \ldots, N-1\}$. Para isso vamos introduzir a seguinte notação, seja $u=\left(u_{1}, u_{2}, \ldots, u_{N}\right) \in \mathbb{C}^{N}$ e $r \in \mathbb{Z}^{N}$ definimos

$$
D(u, r):=t^{r} \sum_{i=1}^{N} u_{i} d_{i}
$$

com esta notação a ação $\operatorname{de} \operatorname{Vect}\left(\mathbb{T}^{N}\right)$ em $T(W, \gamma)$ fica

$$
D(u, r)\left(q^{\mu} \otimes w\right)=\langle u, \mu\rangle q^{\mu+r} \otimes w+q^{\mu+r} \otimes\left(r u^{t}\right) w
$$

onde $\langle.,$.$\rangle é o produto interno usual e \left(r u^{t}\right) \in \mathfrak{g l}_{N}$. Aqui vale a pena notar que $\left(r u^{t}\right) w=\langle u, w\rangle r$ para todo $u, r, w \in \mathbb{C}^{N}$. 
Suponha que exista $M$ um sub-módulo de $q^{\gamma} \Omega^{k}$ tal que $\widehat{W}(\gamma, k)$ seja um submódulo próprio de $M$. Seja $q^{\mu} \otimes\left(v_{1} \wedge \ldots \wedge v_{k}\right) \in M \backslash \widehat{W}(\gamma, k)$. Temos que o conjunto $\left\{\mu, v_{1}, v_{2}, \ldots, v_{k}\right\}$ é 1. i.. Com efeito, sabemos que $\left\{v_{1}, v_{2}, \ldots, v_{k}\right\}$ é linearmente independente, logo suponha que existam $\alpha_{i} \in \mathbb{C}$ tais que $\mu=\sum_{i=1}^{k} \alpha_{i} v_{i}$, e sem perda de generalidade podemos supor $\alpha_{1} \neq 0$. Assim

$$
M \backslash \widehat{W}(\gamma, k) \ni \alpha_{1} q^{\mu} \otimes\left(v_{1} \wedge \ldots \wedge v_{k}\right)=\sum_{i=1}^{k} q^{\mu} \otimes\left(\alpha_{i} v_{i} \wedge \ldots \wedge v_{k}\right)=q^{\mu} \otimes\left(\mu \wedge v_{2} \wedge \ldots \wedge v_{k}\right)
$$

Absurdo, portanto, o conjunto $\left\{\mu, v_{1}, v_{2}, \ldots, v_{k}\right\}$ é linearmente independente, e assim, podemos escolher $u \in \mathbb{C}^{N}$ tal que $\langle u, \mu\rangle \neq 0$ e para todo $i,\left\langle u, v_{i}\right\rangle=0$, e teremos que para todo $r \in \mathbb{Z}^{N}$

$$
D(u, r) q^{\mu} \otimes\left(v_{1} \wedge \ldots \wedge v_{k}\right)=\langle u, \mu\rangle q^{\mu+r} \otimes\left(v_{1} \wedge \ldots \wedge v_{k}\right) \in M
$$

Logo $\left(v_{1} \wedge \ldots \wedge v_{k}\right) \in M_{*}$ e pelo lema 3.3 .1 temos que $M=q^{\gamma} \Omega\left(\mathbb{T}^{N}\right)$, implicando que $\widehat{W}(\gamma, k)$ é maximal.

\subsection{Carácteres dos Submódulos}

Antes de descrevermos os carácteres dos submódulos, trataremos o caso $\gamma=0$ que é quando $\widehat{W}(0, k) \neq W(0, k)$. Temos que no quociente, $\widehat{W}(0, k) / W(0, k)$, Vect $\mathbb{T}^{\bar{N}}$ age trivialmente. Com efeito, $t^{r} d_{a}\left(q^{0} \otimes\left(v_{1} \wedge \ldots \wedge v_{k}\right)\right) \in W(0, k)$ se $r \neq 0$. E se $r=0$ então $d_{a}\left(q^{0} \otimes\left(v_{1} \wedge \ldots \wedge v_{k}\right)\right)=0$. Com isso, temos que o único submódulo irredutível de $q^{\gamma} \Omega^{k}\left(\mathbb{T}^{N}\right)$ é, de fato, $W(\gamma, k)$. Assim concluímos com o seguinte teorema.

Teorema 3.4.1. Para $k \in\{1, \ldots, N-1\}$, o único sub-módulo próprio de $q^{\gamma} \Omega^{k}\left(\mathbb{T}^{N}\right)$ é $W(\gamma, k) e$ seu carácter é dado por

$$
\chi(W(\gamma, k))=\sum_{\substack{\mu \in \gamma+\mathbb{Z}^{N} \\
\mu \neq 0}}\left(\begin{array}{l}
n-1 \\
k-1
\end{array}\right) e^{\mu}
$$

onde, para $\mu \in \gamma+\mathbb{Z}^{N}$, definimos $\mu \in \mathfrak{h}_{\operatorname{Vect}\left(\mathbb{T}^{N}\right)}^{*} \operatorname{como} \mu\left(d_{a}\right)=\mu_{a}$.

Demonstração. Dado $\mu \in \gamma+\mathbb{Z}^{N}$ temos, como definido no enunciado do teorema o peso $\mu \in$ $\mathfrak{h}_{\operatorname{Vect}\left(\mathbb{T}^{N}\right)}^{*}$, e o espaço de peso $V_{\mu}$ é dado por

$$
V_{\mu}=q^{\mu} \otimes\left(\mathbb{C}(\mu) \wedge \mathbb{C}^{N} \wedge \ldots \wedge \mathbb{C}^{N}\right)
$$

Se $\mu=0$ o espaço de peso $V_{\mu}$ tem dimensão zero, caso contrário, podemos, a partir de $\mu$, completar uma base de $\mathbb{C}^{N}$, digamos $\left\{\mu, v_{1}, \ldots, v_{N-1}\right\}$. E a partir desta base, podemos construir a seguinte base de $V_{\mu},\left\{\mu \wedge v_{i_{1}} \wedge \ldots \wedge v_{i_{k-1}} \mid 1 \leq i_{1}<i_{2}<\ldots<i_{k-1} \leq n-1\right\}$. Logo, concluímos que a dimensão de $V_{\mu}$ é $\left(\begin{array}{l}n-1 \\ k-1\end{array}\right)$ 


\section{Capítulo 4}

\section{Superálgebra de Vértice}

Neste capítulo apresentamos as definições e notações de uma superálgebra de vértice e de uma superálgebra vértice de Lie. Apresentamos quatro exemplos que nos serão úteis.

\subsection{Definições e Notações}

Uma estrutura algébrica que será de grande utilidade para nós é a estrutura de álgebra de vértice. A seguir apresentamos sua definição seguindo [11] e [14].

Definição 4.1.1. Uma superálgebra de vértice é um espaço vetorial $V, \mathbb{Z}_{2}$-graduado, com um vetor identidade $\mathbb{1}$, para indicar um estado de vácuo, um operador $D$ chamado de translação infinitesimal no espaço $V$, e uma aplicação linear $Y$ chamada correspondência de estado-campo:

$$
\begin{aligned}
& Y(\cdot, z): V \rightarrow(\operatorname{End} V)\left[\left[z, z^{-1}\right]\right] \\
& a \mapsto Y(a, z)=\sum_{n \in \mathbb{Z}} a_{(n)} z^{-n-1},
\end{aligned}
$$

onde $a_{(n)} \in \operatorname{End}(V)$. Estes devem respeitar o seguinte conjunto de axiomas

1. Para todos $a, b \in V, a_{(n)} b=0$ para $n$ suficientemente grande;

2. $[D, Y(a, z)]=Y(D(a), z)=\frac{d}{d z} Y(a, z)$, para todo $a \in V$;

3. $Y(\mathbb{1}, z)=\operatorname{Id}_{V} z^{0}$, onde $\operatorname{Id}_{V}$ é a identidade em $\operatorname{End}(V)$;

4. $Y(a, z) \mathbb{1} \in V[[z]]$ e $\left.Y(a, z) \mathbb{1}\right|_{z=0}=a$, para todo $a \in V$;

5. Para todos $a, b \in V$ os campos $V(a, z)$ e $V(b, w)$ são mutualmente locais, isto é,

$$
(z-w)^{n}[Y(a, z), Y(b, w)]=0, \quad \text { para } n \text { suficientemente grande. }
$$

Uma superálgebra de vértice é chamada de conforme ou pela sigla VOA (Vertex Operator Algebra), se em adição, possuir um elemento $\omega$, chamado de elemento de Virasoro, tal que

6. As componentes $L_{n}:=\omega_{(n+1)}$ do campo

$$
Y(\omega, z)=\sum_{n \in \mathbb{Z}} \omega_{(n)} z^{-n-1}=\sum_{n \in \mathbb{Z}} L_{n} z^{-n-2},
$$

satisfazem a relação de Virasoro

$$
\left[L_{m}, L_{n}\right]=(m-n) L_{m+n}+\frac{C_{V i r}}{12}\left(m^{3}-m\right) \delta_{m+n, 0},
$$


onde $C_{V i r}$ age em $V$ pela multiplicação de um escalar chamado de carga central de $V$;

7. $D=L_{-1}$;

8. O operador $L_{0}$ é diagonalizável em $V$.

Uma propriedade que é consequência dos axiomas é a seguinte fórmula

$$
\left[Y\left(a, z_{1}\right), Y\left(b, z_{2}\right)\right]=\sum_{n>0} \frac{1}{n !} Y\left(a_{(n)^{b}} b, z_{2}\right)\left[z_{1}^{-1}\left(\frac{\partial}{\partial z_{2}}\right)^{n} \delta\left(\frac{z_{2}}{z_{1}}\right)\right],
$$

onde $\delta$ é a função dada por

$$
\delta(z)=\sum_{n \in \mathbb{Z}} z^{n}
$$

O produto entre dois campos não necessariamente está bem definido. Para isso é definimos o produto ordenado normal que estará sempre bem definido. Dado um campo $a(z)=\sum_{j \in \mathbb{Z}} a_{(j)} z^{-j-1}$ definimos sua parte positiva e negativa como

$$
a(z)_{-}=\sum_{j=0}^{\infty} a_{(j)} z^{-j-1}, \quad a(z)_{+}=\sum_{j=-1}^{-\infty} a_{(j)} z^{-j-1}
$$

e o produto ordenado normal por

$$
: a(z) b(z)::=a(z)_{+} b(z)+(-1)^{p(a) p(b)} b(z) a(z)_{-}
$$

onde $p(a), p(b) \in\{0,1\}$ são as paridades dos campos $a(z)$ e $b(z)$, respectivamente.

\subsection{Superálgebras de Lie de Vértice}

Seja $\mathcal{L}$ uma superálgebra de Lie com base $\left\{u_{(n)}, c_{(-1)} \mid u \in \mathcal{U}, c \in \mathcal{C}, n \in \mathbb{Z}\right\}(\mathcal{U}$ e $\mathcal{C}$ são conjunto de índices). Definamos os seguintes campos em $\mathcal{L}\left[z, z^{-1}\right]$ :

$$
u(z)=\sum_{n \in \mathbb{Z}} u_{(n)} z^{-n-1}, \quad c(z)=c_{(-1)} z^{0}, \quad u \in \mathcal{U}, c \in \mathcal{C} .
$$

Seja $\mathcal{F}$ o subespaço de $\mathcal{L}\left[\left[z, z^{-1}\right]\right]$ gerado por todos os campos $u(z), c(z)$ e suas derivadas de todas as ordens.

Definição 4.2.1. Sejam $\mathcal{L}$ uma superálgebra de Lie e $\mathcal{F}$ o subespaço como definidos anteriormente, diremos que $\mathcal{L}$ é uma superálgebra de vértice de Lie se os seguintes axiomas valem:

(VL1) para todo $\mathrm{x}, \mathrm{y} \in \mathcal{U}$,

$$
\left[x\left(z_{1}\right), y\left(z_{2}\right)\right]=\sum_{j=0}^{n} f_{j}\left(z_{2}\right)\left[z^{-1}\left(\frac{\partial}{\partial z_{2}}\right)^{j} \delta\left(\frac{z_{2}}{z_{1}}\right)\right],
$$

onde $f_{j}(z) \in \mathcal{F}, n \leq 0$ e depende de $x, y$.

(VL2) para todo $c \in \mathcal{C}$, os elementos $c_{(-1)}$ são centrais em $\mathcal{L}$.

Seja $\mathcal{L}_{(+)}$o subespaço de $\mathcal{L}$ com base $\left\{u_{(n)} \mid u \in \mathcal{U}, n \geq 0\right\}$ e seja $\mathcal{L}_{(-)}$o subespaço de $\mathcal{L}$ com base $\left\{u_{(n)}, c_{(-1)} \mid u \in \mathcal{U}, c \in \mathcal{C}, n<0\right\}$

A álgebra de vértice envelopante universal, $V_{\mathcal{L}}$, de uma superálgebra de vértice de Lie $\mathcal{L}$ é definida como o módulo

$$
V_{\mathcal{L}}=\operatorname{Ind}_{\mathcal{L}_{(+)}}^{\mathcal{L}}(\mathbb{C} \mathbb{1}) \simeq U\left(\mathcal{L}_{(-)}\right) \otimes \mathbb{1},
$$

onde $\mathbb{C} \mathbb{1}$ é um $\mathcal{L}_{(+)}$-módulo trivial de dimensão 1. 
O seguinte teorema, presente em [6], mostra como um superálgebra de vértice de Lie $\mathcal{L}$ gera um superálgebra de vértice $V_{\mathcal{L}}$ e uma outra superálgebra de vértice que é quociente desta.

Teorema 4.2.2. Seja $\mathcal{L}$ uma superálgebra de vértice de Lie. Então

(a) $V_{\mathcal{L}}$ tem estrutura de superálgebra de vértice com o vetor identidade $\mathbb{1}$, a translação infinitesimal $D$ será dada por $D\left(u_{(n)}\right)=-n u_{(n-1)}, D\left(c_{(-1)}=0, u \in \mathcal{U}, c \in \mathcal{C}\right.$, e a correspondência estado-campo é definida por:

$$
\begin{gathered}
Y\left(a_{\left(-1-n_{1}\right)}^{1} \ldots a_{\left(-1-n_{k-1}\right)}^{k-1} a_{\left(-1-n_{k}\right)}^{k} \mathbb{1}, z\right)= \\
=:\left(\frac{1}{n_{1} !}\left(\frac{\partial}{\partial z}\right)^{n_{1}} a^{1}(z)\right) \ldots:\left(\frac{1}{n_{k-1} !}\left(\frac{\partial}{\partial z}\right)^{n_{k-1}} a^{k-1}(z)\right)\left(\frac{1}{n_{k} !}\left(\frac{\partial}{\partial z}\right)^{n_{k}} a^{k}(z)\right): \ldots:,
\end{gathered}
$$

(b) Todo módulo limitado de $\mathcal{L}$ é um módulo de $V_{\mathcal{L}}$.

(c) Para um carácter arbitrário $\chi: \mathcal{C} \rightarrow \mathbb{C}$, o módulo quociente

$$
V_{\mathcal{L}}(\gamma)=U\left(\mathcal{L}_{(-)}\right) \mathbb{1} / U\left(\mathcal{L}_{(-)}\right)\left\langle\left(c_{(-1)}-\chi(c)\right) \mathbb{1}\right\rangle_{c \in \mathcal{C}}
$$

é uma superálgebra de vértice. $O$ valor $\chi(c)$, também, é chamado de carga central.

(d) Qualquer módulo para $\mathcal{L}$ no qual $c_{(-1)}$ age como $\chi(c) \mathrm{Id}$, para todo $c \in \mathcal{C}$, é um módulo para superálgebra de vértice $V_{\mathcal{L}}(\gamma)$.

Apresentamos agora quatro exemplos de VOAs que serão de grande importância no que se segue do texto, além de mostrar a utilidade deste último teorema para obtenção de VOAs a partir de álgebras de vértice de Lie.

VOA Hiperbólico: Um reticulado hiperbólico, Hyp, é um grupo abeliano com $2 N$ geradores $\left\{u^{a}, v^{a} \mid a=1, \ldots, N\right\}$ e a forma bilinear simétrica

$$
(\cdot \mid \cdot): \text { Hyp } \times \text { Hyp } \rightarrow \mathbb{Z}
$$

definida como

$$
\left(u^{a} \mid v^{b}\right)=\delta_{a b}, \quad\left(u^{a} \mid u^{b}\right)=\left(v^{a} \mid v^{b}\right)=0, \quad a, b=1, \ldots, N .
$$

Podemos complexificar Hyp para obter um espaço vetorial de dimensão $2 \mathrm{~N}$ sobre $\mathbb{C}$,

$$
\mathcal{H}:=\operatorname{Hyp} \otimes_{\mathbb{Z}} \mathbb{C} .
$$

Podemos afinizar $\mathcal{H}$ definindo

$$
\widehat{\mathcal{H}}:=\mathbb{C}\left[t, t^{-1}\right] \otimes \mathcal{H} \oplus \mathbb{C} K,
$$

com o colchete dado por

$$
\begin{gathered}
{\left[x_{(n):} y_{(m)}\right]=n(x \mid y) \delta_{n,-m} K, \quad x, y \in \mathcal{H},} \\
{[\widehat{\mathcal{H}}, K]=0,}
\end{gathered}
$$

onde $x_{(n)}=t^{n} \otimes x$.

Com relação ao grau de $t$, temos a seguinte decomposição triangular de $\widehat{\mathcal{H}}=\widehat{\mathcal{H}}_{-} \oplus \widehat{\mathcal{H}}_{0} \ominus \widehat{\mathcal{H}}_{+}$, onde $\widehat{\mathcal{H}}_{0}:=1 \otimes \mathcal{H} \oplus \mathbb{C} K$ e $\widehat{\mathcal{H}}_{ \pm}:=t^{ \pm 1} \mathbb{C}\left[t^{ \pm 1}\right] \otimes \mathcal{H}$

Seja Hyp ${ }^{+}$o subreticulado de Hyp gerado por $\left\{u^{a} \mid a=1, \ldots, N\right\}$. Consideremos a sua álgebra de grupo $\mathbb{C}\left[\mathrm{Hyp}^{+}\right]=\mathbb{C}\left[e^{ \pm u^{1}}, \ldots, e^{ \pm u^{N}}\right]$, podemos definir nesta álgebra uma ação de $\widehat{\mathcal{H}}_{0} \oplus \widehat{\mathcal{H}}_{+}$:

$$
x_{(0)} e^{y}=(x \mid y) e^{y}, \quad K e^{y}=e^{y}, \quad \widehat{\mathcal{H}}_{+} e^{y}=0 .
$$

Definimos o seguinte módulo induzido

$$
V_{\mathrm{Hyp}}^{+}:=\operatorname{Ind}_{\widehat{\mathcal{H}}_{0} \oplus \widehat{\mathcal{H}}_{+}}^{\hat{\mathcal{H}}}\left(\mathbb{C}\left[\operatorname{Hyp}^{+}\right]\right) .
$$


Temos que $V_{\text {Hyp }}^{+}$pode ser realizado como um espaço de Fock sobre $\widehat{\mathcal{H}}$

$$
V_{\mathrm{Hyp}}^{+}=\mathbb{C}\left[q_{1}^{ \pm 1}, q_{2}^{ \pm 1}, \ldots, q_{N}^{ \pm 1}\right] \otimes \mathbb{C}\left[u_{p j},\left.v_{p j}\right|_{j=1,2, \ldots} ^{p=1, \ldots, N}\right],
$$

com a ação de $\widehat{\mathcal{H}}$ dada por

$$
\begin{gathered}
u_{(-j)}^{p}=j u_{p j}, \quad u_{(j)}^{p}=\frac{\partial}{\partial v_{p j}}, \quad u_{(0)}^{p}=0, \\
v_{(-j)}^{p}=j v_{p j}, \quad v_{(j)}^{p}=\frac{\partial}{\partial u_{p j}}, \quad v_{(0)}^{p}=q_{p} \frac{\partial}{\partial q_{p}} .
\end{gathered}
$$

O espaço $V_{\text {Hyp }}^{+}$admite uma estrutura de álgebra de vértice. A correspondência estado-campo é dada, nos geradores, por

$$
\begin{gathered}
Y\left(u_{p 1}, z\right)=u^{p}(z)=\sum_{j \in \mathbb{Z}} u_{(j)}^{p} z^{-j-1} \\
Y\left(v_{p 1}, z\right)=v^{p}(z)=\sum_{j \in \mathbb{Z}} v_{(j)}^{p} z^{-j-1}, \quad p=1, \ldots, N, \\
Y\left(q^{r}, z\right)=q^{r} \exp \left(\sum_{p=1}^{N} r_{p} \sum_{j=1}^{\infty} \frac{z^{j}}{j} u_{(-j)}^{p}\right) \exp \left(-\sum_{p=1}^{N} r_{p} \sum_{j=1}^{\infty} \frac{z^{-j}}{j} u_{(j)}^{p}\right) .
\end{gathered}
$$

O elemento de Virasoro de $V_{\text {Hyp }}^{+}$é dado por

$$
\omega^{\mathrm{Hyp}}=\sum_{p=1}^{N} u_{p 1} v_{p 1},
$$

o campo de Virasoro

$$
Y\left(\omega^{\mathrm{Hyp}}, z\right)=\sum_{p=1}^{N}: u^{p}(z) v^{p}(z):
$$

e a sua carga central é $-2 N$.

Fixando $\gamma \in \mathbb{C}^{N}$, o espaço

$$
M_{\mathrm{Hyp}}(\gamma):=q^{\gamma} \mathbb{C}\left[q_{1}^{ \pm 1}, q_{2}^{ \pm 1}, \ldots, q_{N}^{ \pm 1}\right] \otimes \mathbb{C}\left[u_{p j},\left.v_{p j}\right|_{j=1,2, \ldots} ^{p=1, \ldots, N}\right]
$$

é um módulo irredutível para $V_{\text {Hyp }}^{+}$.

VOA $\widehat{g l}_{N}$ afim: Como $\mathfrak{g l}_{n}$ é redutível, porém não simples, esta álgebra admite mais de uma maneira de ser afinizada. Aqui consideramos a seguinte versão de $\widehat{\mathfrak{g l}}_{N}$ :

$$
\widehat{\mathfrak{g l}}_{N}=\mathbb{C}\left[t, t^{-1}\right] \otimes \mathfrak{g l}_{n} \oplus \mathbb{C} C,
$$

com o colchete definido como

$$
\left[t^{n} \otimes X, t^{m} \otimes Y\right]=t^{n+m} \otimes[X, Y]+n \delta_{n,-m} \operatorname{Tr}(X Y) C .
$$

Observe que $\widehat{\mathfrak{g l}}_{n}$ é uma álgebra de Lie de vértice e considere sua álgebra de vértice envelopante universal $V_{\mathrm{gl}_{n}}$ com carga central 1 .

A correspondência espaço-campo é dada nos geradores por:

$$
Y\left(X_{(-1)} \mathbb{1}, z\right)=X(z)=\sum_{j \in \mathbb{Z}} X_{(j)} z^{-j-1}, \quad X \in g l_{N}
$$


onde $X_{(j)}=\operatorname{ad}\left(t^{j} \otimes X\right)$.

Como $\mathfrak{g l}_{N}$ admite a decomposição $\mathfrak{g l}_{N}=\mathfrak{s l}_{N} \oplus \mathbb{C} I$, onde $I$ é a matriz identidade, temos que a álgebra de vértice afim $\widehat{\mathfrak{g l}}_{N}$ é o produto tensorial da álgebra de vértice afim $\widehat{\mathfrak{s l}}_{N}$ com a álgebra de vértice de Heisenberg. O elemento de Virasoro $\omega^{\mathfrak{g l}_{N}}$ de $V_{\widehat{\mathfrak{g l}} l_{N}}$ é a soma dos elementos de Virasoro $\omega^{\mathfrak{s l}}{ }_{N}$ e $\omega^{\text {Hei }}$ de $\widehat{\mathfrak{s l}}_{N}$ e da álgebra de vértice de Heisenberg.

Em [2] foram escolhidos os seguintes elementos de Virasoro:

$$
\begin{gathered}
\omega^{\mathbf{s l}}=\frac{1}{2(N+1)}\left(\sum_{i, j=1}^{N} E_{(-1)}^{i j} E_{(-1)}^{j i} \mathbb{1}-\frac{1}{N} I_{(-1)} I_{(-1)} \mathbb{1}\right), \\
\omega^{\text {Hei }}=\frac{1}{2 N} I_{(-1)} I_{(-1)} \mathbb{1}+\frac{1}{2} I_{(-2)} \mathbb{1}
\end{gathered}
$$

As cargas centrais são $N-1$ e $1-3 N$, respectivamente.

Adicionando estes dois elementos obtemos como elemento de Virasoro para $V_{\widehat{\mathfrak{g}}_{N}}$ :

$$
\omega^{\mathfrak{g l}_{N}}=\frac{1}{2(N+1)}\left(\sum_{i, j=1}^{N} E_{(-1)}^{i j} E_{(-1)}^{j i} \mathbb{1}+I_{(-1)} I_{(-1)} \mathbb{1}\right)+\frac{1}{2} I_{(-2)} \mathbb{1} .
$$

com carga central $-2 N$.

Seja $W$ um $\mathfrak{g l}_{N}$ módulo simples de dimensão finita. Definindo a ação de $C$ como a ação pela identidade e $\left(t \mathbb{C}[t] \otimes \mathfrak{g l}_{N}\right) W=0$, podemos então definir um módulo de Verma generalizado para $\widehat{\mathfrak{g l}}_{N}$ e considere seu submódulo irredutível $L_{\mathfrak{g l}}(W)$. Então $L_{\mathfrak{g} l_{N}}(W)$ é um módulo irredutível para $V_{\mathfrak{g l}}$.

VOA Virasoro: A álgebra de Virasoro (veja seção 2.2) é uma álgebra de vértice de Lie com $\mathcal{U}=\{\omega\}$ e $\mathcal{C}=\{c\}$ onde

$$
\omega(z)=\sum_{j \in \mathbb{Z}} \omega_{(j)}^{V i r} z^{-j-1}=\sum_{j \in \mathbb{Z}} L_{j} z^{-j-2}
$$

Seja $V_{\text {Vir }}$ a sua álgebra de vértice envelopante universal com carga central zero, $\chi(c)=0$. E seja $L_{\mathrm{Vir}}(h), h \in \mathbb{C}$ o módulo irredutível de peso máximo $v_{h}$ para a álgebra de Lie de Virasoro com $L_{0} v_{h}=h v, c v_{h}=0$.

VOA reticulado euclidiano $\mathbb{Z}^{N}$ : Considere a superálgebra $C l_{N}$ com base $\left\{\phi_{(j)}^{p}, \psi_{(j)}^{p} \mid p=1, \ldots, N\right.$, $j \in \mathbb{Z}\}$ para sua parte impar e como base para sua parte par o elemento central K. O colchete de Lie é dado por

$$
\left[\phi_{(m)}^{a}, \psi_{(n)}^{b}\right]=\delta_{a b} \delta_{m,-n-1} K, \quad\left[\phi_{(m)}^{a}, \phi_{(n)}^{b}\right]=\left[\psi_{(m)}^{a}, \psi_{(n)}^{b}\right]=0
$$

Considere os seguintes campos:

$$
\phi^{a}(z)=\sum_{j \in \mathbb{Z}} \phi_{(j)}^{a} z^{-j}, \quad \psi^{a}(z)=\sum_{j \in \mathbb{Z}} \psi_{(j)}^{a} z^{-j}, \quad K(z)=K z^{0} .
$$

A superálgebra de vértice do reticulado euclidiano, $V_{\mathbb{Z}^{N}}$, é isomorfa a álgebra de vértice envelopante universal de $C l_{N}$ com carga central 1 . Como espaço vetorial ele é o único $C l_{N}$-módulo gerado pelo vetor de vacuidade $\mathbb{1}$, satisfazendo

$$
K \mathbb{1}=\mathbb{1}, \quad \phi_{(j)}^{p} \mathbb{1}=\psi_{(j)}^{p} \mathbb{1}=0, \text { para } j \geq 0, p=1, \ldots, N .
$$

Em sua realização fermionica $V_{\mathbb{Z}^{N}}$ é a álgebra exterior com geradores $\left\{\phi_{(j)}^{p},\left.\psi_{(j)}^{p}\right|_{j \leq-1} ^{p=1, \ldots, N}\right\}$. A correspondencia estado-campo é dada pela fórmula do teorema 4.2.2 item (a). 
O elemento de Virasoro é dado por:

$$
\omega^{f e r}=\sum_{p=1}^{N} \phi_{(-2)}^{p} \psi_{(-1)}^{p} \mathbb{1},
$$

sua carga central é $-2 N$. 


\section{Capítulo 5}

\section{Módulos Limitados}

Neste capítulo é apresentada uma classe de módulos para álgebra $\operatorname{Vect}\left(\mathbb{T}^{N}\right)$ e a classificação dos módulos irredutíveis desta classe. Para isso, é utilizada a representação de campo livre para a álgebra $\mathcal{D} \ltimes \mathcal{K}$ e depois é feita a restrição a álgebra $\operatorname{Vect}\left(\mathbb{T}^{N}\right)$. Para verificar que certos módulos são redutíveis utilizamos o Chiral de de Rham.

\subsection{Módulos Induzidos}

Consideremos a álgebra $\mathcal{D}=\operatorname{Vect}\left(\mathbb{T}^{N+1}\right)$, que é a álgebra de campos vetoriais de um toro $N+1$ dimensional, com as coordenadas $t_{0}, t_{1}, \ldots, t_{N}$, onde $t_{0}$ é a coordenada especial. Consideremos a graduação em $\mathbb{Z}$ com relação ao grau $t_{0}$, isto induz a seguinte decomposição triangular

$$
\mathcal{D}=\mathcal{D}_{-} \oplus \mathcal{D}_{0} \oplus \mathcal{D}_{+}
$$

onde $\mathcal{D}_{+}$e $\mathcal{D}_{-}$são as subálgebras com o grau de $t_{0}$ positivo e negativo, respectivamente, e $\mathcal{D}_{0}$ a subálgebra com $t_{0}$ com grau zero, vale notar que $\mathcal{D}_{0}$ é a soma semi-direta da álgebra $\operatorname{Vect}\left(\mathbb{T}^{N}\right)$ com o ideal abeliano $\mathbb{C}\left[t_{1}^{ \pm 1}, \ldots, t_{N}^{ \pm 1}\right] d_{0}$.

Considere o Vect $\left(\mathbb{T}^{n}\right)$-módulo tensorial $T(W, \gamma)$, podemos estender sua ação a $\mathcal{D}_{0} \oplus \mathcal{D}_{+}$com $\mathbb{C}\left[t_{1}^{ \pm 1}, \ldots, t_{N}^{ \pm 1}\right] d_{0}$ agindo por deslocamentos, isto é,

$$
t^{r} d_{0}\left(q^{\mu} \otimes w\right)=\beta q^{\mu+r} \otimes w,
$$

e $\mathcal{D}_{+}$agindo trivialmente. Este módulo será denotado por $T=T(W, \gamma, \beta)$.

Por fim, induzimos para todo $\mathcal{D}$ :

$$
M(T)=\operatorname{Ind}_{\mathcal{D}_{0} \oplus \mathcal{D}_{+}}^{\mathcal{D}} T \simeq \mathcal{U}\left(\mathcal{D}_{-}\right) \otimes T .
$$

Este módulo tem uma decomposição em espaços de peso com relação a álgebra de Cartan $\left\langle d_{0}, d_{1}, \ldots\right.$ , $\left.d_{N}\right\rangle$. No entanto, todos os espaços de peso de $M(T)$, além do próprio $T$, são de dimensão infinita.

Porém, um teorema de [1] nos garante que no quociente irredutível os espaços de peso têm dimensão finita.

Teorema 5.1.1. (i) O módulo $M(T)$ tem um único submódulo maximal $M^{\text {rad }}$.

(ii) O quociente irredutivel $L(T)=M / M^{\text {rad }}$ tem espaços de peso de dimensão finita.

Para entender a estrutura dos módulos $L(T)$ consideramos uma categoria de módulos para álgebra toroidal completa descrita em [2]. Se considerarmos $\dot{g}=0$ obtemos uma categoria de módulos para $\mathcal{K} \rtimes \mathcal{D}$. Veremos a seguir que quando restringimos à ação de $\mathcal{D}$ esses módulos continuarão irredutíveis e daremos o carácter destes módulos. No entanto, antes de utilizarmos este resultado devemos verificar se $\mathcal{K} \rtimes \mathcal{D}$ é uma superálgebra de vértice de Lie.

Teorema 5.1.2. A álgebra $\mathcal{D} \ltimes \mathcal{K}$ é uma superálgebra de vértice de Lie. 
Demonstrą̧ão. Considere os seguintes campos

$$
\begin{aligned}
k_{0}(r, z) & =\sum_{j \in \mathbb{Z}} t_{0}^{j} t^{r} k_{0} z^{-j}, & k_{a}(r, z) & =\sum_{j \in \mathbb{Z}} t_{0}^{j} t^{r} k_{a} z^{-j-1}, \\
d_{a}(r, z) & =\sum_{j \in \mathbb{Z}} t_{0}^{j} t^{r} d_{a} z^{-j-1}, & d_{0}(r, z) & =\sum_{j \in \mathbb{Z}} t_{0}^{j} t^{r} d_{0} z^{-j-2} .
\end{aligned}
$$

0 conjunto $C$ consiste de um único elemento que corresponde ao campo central $k_{0}(0, z)$, assim, (VL2) vale. Para verificar (VL1) devemos calcular os colchetes dos demais campos. Temos

$$
\begin{gathered}
{\left[k_{a}\left(r, z_{1}\right), k_{b}\left(s, z_{2}\right)\right]=0,} \\
{\left[d_{a}\left(r, z_{1}\right), d_{b}\left(s, z_{2}\right)\right]=\left[\sum_{i \in \mathbb{Z}} t_{0}^{i} t^{r} d_{a} z_{1}^{-i-1}, \sum_{j \in \mathbb{Z}} t_{0}^{j} t^{s} d_{b} z_{2}^{-j-1}\right]=\sum_{i, j \in \mathbb{Z}}\left[t_{0}^{i} t^{r} d_{a} z_{1}^{-i-1}, t_{0}^{j} t^{s} d_{b} z_{2}^{-j-1}\right]=} \\
=\sum_{i, j \in \mathbb{Z}}\left(s_{a} t_{0}^{i+j} t^{r+s} d_{b}-r_{b} t_{0}^{i+j} t^{r+s} d_{a}\right) z_{1}^{-i-1} z_{2}^{-j-1}=\sum_{i, k \in \mathbb{Z}}\left(s_{a} t_{0}^{k} t^{r+s} d_{b} z_{2}^{-k-1}-r_{b} t_{0}^{k} t^{r+s} d_{a} z_{2}^{-k-1}\right) z_{1}^{-i-1} z_{2}^{i}= \\
=\left(s_{a} d_{b}\left(r+s, z_{2}\right)-r_{b} d_{a}\left(r+s, z_{2}\right)\right)\left[z_{1}^{-1} \delta\left(\frac{z_{2}}{z_{1}}\right)\right] \\
{\left[d_{0}\left(r, z_{1}\right), \dot{d}_{0}\left(s, z_{2}\right)\right]=\sum_{i, j \in \mathbb{Z}}\left[t_{0}^{i} t^{r} d_{0} z_{1}^{-i-2}, t_{0}^{j} t^{s} d_{0} z_{2}^{-j-2}\right]=\sum_{i, j \in \mathbb{Z}}(j-i)\left(t_{0}^{i+j} t^{r+s} d_{0}\right) z_{1}^{-i-2} z_{2}^{-j-2}=} \\
=\sum_{i, k \in \mathbb{Z}}(k-2 i)\left(t_{0}^{k} t^{r+s} d_{0}\right) z_{1}^{-i-2} z_{2}^{-k+i-2}=\sum_{i, k \in \mathbb{Z}}(k+2)\left(t_{0}^{k} t^{r+s} d_{0} z_{2}^{-k-3}\right)\left(z_{1}^{-1} \frac{z_{2}^{i+1}}{z_{1}^{i+1}}\right)- \\
-2 \sum_{i, k \in \mathbb{Z}}(i+1)\left(t_{0}^{k} t^{r+s} d_{0} z_{2}^{-k-2}\right)\left(z_{1}^{-1} \frac{z_{2}^{i}}{z_{1}^{i+1}}\right)=-\left(\frac{\partial}{\partial z_{2}} d_{0}\left(r+s, z_{2}\right)\right)\left[z_{1}^{-1} \delta\left(\frac{z_{2}}{z_{1}}\right)\right]- \\
\left.-2 d_{0}\left(r+s, z_{2}\right)\right)\left[z_{1}^{-1} \frac{\partial}{\partial z_{2}} \delta\left(\frac{z_{2}}{z_{1}}\right)\right] .
\end{gathered}
$$

Logo estes colchetes estão no formato de (VL1).

Concluímos esta seção com o teorema de [2] que nos dá a representação de campo livre para a álgebra $\mathcal{D} \ltimes \mathcal{K}$.

Teorema 5.1.3. (i) Sejam $M_{\mathrm{Hyp}}, M_{\mathfrak{g l}_{N}}$ e $M_{V i r}$ módulos para $V_{\mathrm{Hyp}}^{+}, V_{\mathfrak{g l}_{N}}$ e $V_{V i r}$, respectivamente. Então o produto tensorial

$$
\mathcal{M}=M_{\mathrm{Hyp}} \otimes M_{\mathfrak{g l}_{N}} \otimes M_{V i r}
$$

é um módulo para a álgebra $\mathcal{D} \ltimes \mathcal{K}$, com ação dada por:

$$
\begin{gathered}
\sum_{j \in \mathbb{Z}} t_{0}^{j} t^{r} k_{0} z^{-j}=k_{0}(r, z) \rightarrow Y\left(q^{r}, z\right), \\
\sum_{j \in \mathbb{Z}} t_{0}^{j} t^{r} k_{a} z^{-j-1}=k_{a}(r, z) \rightarrow u^{a}(z) Y\left(q^{r}, z\right) \\
\sum_{j \in \mathbb{Z}} t_{0}^{j} t^{r} d_{a} z^{-j-1}=d_{a}(r, z) \rightarrow: v^{a}(z) Y\left(q^{r}, z\right):+\sum_{p=1}^{n} r_{p} E^{p a}(z) Y\left(q^{r}, z\right), \\
\sum_{j \in \mathbb{Z}} t_{0}^{j} t^{r} d_{0} z^{-j-2}=d_{0}(r, z) \rightarrow-: Y(\omega, z) Y\left(q^{r}, z\right):-\sum_{i, j=1}^{n} r_{i} u^{j}(z) E^{i j}(z) Y\left(q^{r}, z\right)+
\end{gathered}
$$




$$
+\sum_{p=1}^{n} r_{p}\left(\frac{d}{d z} u^{p}(z)\right) Y\left(q^{r}, z\right)
$$

(ii) O módulo

$$
L(W, \gamma, h)=M_{\mathrm{Hyp}} \otimes L_{g l_{n}} \otimes L_{V i r}
$$

é um módulo irredutível sobre $\mathcal{D} \ltimes \mathcal{K}$.

\subsection{Carácter dos Módulos Induzidos}

Em [4] foi calculado o carácter dos módulos $L(W, \gamma, h)$ para $N=1$. Inicialmente, foi feita a restrição destes módulos para a subálgebra $\widetilde{s l}_{2}=\mathbb{C}\left[t_{0}^{ \pm 1}\right] \otimes s l_{2}$ e verificado o seguinte isomorfismo $L(W, \gamma, h) \simeq U\left(\widetilde{s l}_{2}\right) \otimes T$. Em outras palavras, $L(W, \gamma, h)$ é um módulo de Verma generalizado para $\widetilde{s l}$.

Para os casos $N>1$, temos o seguinte problema, um módulo de Verma generalizado para $\widetilde{s l}_{N+1}$ não pode, em geral, ter sua ação estendida para Vect $\left(\mathbb{T}^{N+1}\right)$. Para contornar isto, em [3] foi dada a definição de módulo generalizado de Wakimoto: $\mathcal{M}$ é um módulo generalizado de Wakimoto com topo $T$ para $\widetilde{s l}_{N+1}$ se $T$ é um $s l_{N+1}$ submódulo com $\widetilde{s l}_{N+1}^{+}$agindo trivialmente (a graduação de $\widetilde{s l} l_{N+1}$ é, também, dada com relação ao grau de $t_{0}$ ), e por fim, o carácter de $\mathcal{M}$ coincide com o carácter de um módulo de Verma generalizado com topo $T$, isto é,

$$
\chi(\mathcal{M})=\chi\left(U\left(\tilde{s l}_{N+1}^{-}\right)\right) \chi(T) .
$$

Teorema 5.2.1. Seja $W$ um $g l_{N}$-módulo irredutível de dimensão finita. $E$ seja $M_{g l_{N}}(W)$ o módulo de Verma induzido de $W$ para $\widehat{g l}_{N}$ com carga central 1 . Então o módulo

$$
\mathcal{M}=q^{\gamma} \mathbb{C}\left[q_{1}^{ \pm 1}, \ldots, q_{N}^{ \pm 1}\right] \otimes \mathbb{C}\left[u_{p j}, v_{p j}\right] \otimes M_{g l_{N}}(W)
$$

é um módulo generalizado de Wakimoto para $\widetilde{s l}_{N+1}$.

Demonstração. Pelo teorema 5.1.3 temos que $\mathcal{M}$ é um $\operatorname{Vect}\left(\mathbb{T}^{N+1}\right)$, por restrição temos que $\mathcal{M}$ é um módulo para $\widetilde{\mathfrak{s}}_{N+1}$ com topo

$$
T(W, \gamma)=q^{\gamma} \mathbb{C}\left[q_{q}^{ \pm 1}, \ldots, q_{N}^{ \pm 1}\right] \otimes W .
$$

O cálculo do carácter de $\mathbb{C}\left[u_{p j}, v_{p j}\right]$ é um difícil problema de combinatória, seu resultado, como pode ser visto em [3], é

$$
\chi\left(\mathbb{C}\left[u_{p j}, v_{p j}\right]\right)=\prod_{k=1}^{\infty}\left(1-s^{k}\right)^{-2 N} .
$$

Vamos calcular e conferir o começo desta série para o caso $N=1$. Lembrando que $\mathbb{C}\left[u_{p j}, v_{p j}\right]$ é realizado como espaço de Fock, isto é, a graduação de $u_{p j}$ e $v_{p j}$ é $j$, temos a seguinte base para esta graduação:

- $\{1\} \Rightarrow \operatorname{dim} 1$

- $\left\{u_{1}, v_{1}\right\} \Rightarrow \operatorname{dim} 2$

- $\left\{u_{1}^{2}, u_{1} v_{1}, v_{1}^{2}, u_{2}, v_{2}\right\} \Rightarrow \operatorname{dim} 5$

- $\left\{u_{1}^{3}, u_{1}^{2} v_{1}, u_{1} v_{1}^{2}, v_{1}^{3}, u_{1} u_{2}, u_{1} v_{2}, u_{2} v_{1}, v_{1} v_{2}, u_{3}, v_{3}\right\} \Rightarrow \operatorname{dim} 10$ 
Observe que $(1-s)^{-2}=\left((1-s)^{-1}\right)^{\prime}$. Expandindo em série $(1-s)^{-1}$ temos

$$
\frac{1}{1-s}=1+s+s^{2}+\ldots \Rightarrow \frac{1}{(1-s)^{-2}}=\left(\frac{1}{1-s}\right)^{\prime}=1+2 s+3 s^{2}+\ldots
$$

$\log 0$

$\prod_{k=1}^{\infty}\left(1-s^{k}\right)^{-2}=\left(1+2 s+3 s^{2}+4 s^{3}+\ldots\right)\left(1+2 s^{2}+\ldots\right)\left(1+2 s^{3}+\ldots\right)=1+2 s+5 s^{2}+10 s^{3}+\ldots$

Comparando os caracteres temos

$\chi\left(\mathbb{C}\left[u_{p j}, v_{p j}\right]\right) \otimes \chi\left(U\left(\hat{\mathfrak{g}}_{N}^{-}\right)\right)=\prod_{k=1}^{\infty}\left(1-s^{k}\right)^{-2 N}+\prod_{k=1}^{\infty}\left(1-s^{k}\right)^{-N^{2}}=\prod_{k=1}^{\infty}\left(1-s^{k}\right)^{-(N+1)^{2}+1}=\chi\left(U\left(\tilde{\mathfrak{s l}}_{N+1}^{-}\right)\right)$.

\subsection{Critério para Irredutibilidade}

Um vetor em $L(W, \gamma, h)$ é dito crítico se é aniquilado por $\mathcal{D}_{+}$. Para um módulo $L(W, \gamma, h)$ ser irredutível devemos então verificar as seguintes condições:

(C) Todo vetor crítico de $L(W, \gamma, h)$ pertence ao seu topo $T(W, \gamma, h)$.

(G) $L(W, \gamma, h)$ é gerado pelo seu topo $T(W, \gamma, h)$ como um módulo sobre $\mathcal{D}_{-}$.

Teorema 5.3.1. Seja $W$ um gl $l_{N}$-módulo irredutivel de dimensão finita, $\gamma \in \mathbb{C}^{N}, h \in \mathbb{C}$. Todo vetor crítico do módulo $L(W, \gamma, h)$ pertence ao seu topo a menos que $h=0$ e $W$ é um módulo trivial para $s_{N}$ com a identidade agindo por $\alpha=N-m N, m=1,2, \ldots$, ou o peso máximo de $W$ é um peso fundamental $\omega_{k}, k=1, \ldots, k-1$, com a identidade agindo por $\alpha=k-m N$.

Como a prova é demasiadamente longa e requer ferramentas que não foram introduzidas, apresentamos aqui uma ideia da prova. O capítulo 8 de [3] é dedicado a construção de uma forma bilinear contra-gradiente não degenerada de

$$
L(W, \gamma, h) \times L\left(W^{*}, \gamma, h\right) \rightarrow \mathbb{C}
$$

onde $W^{*}$ é o espaço dual de $W$ com a estrutura de $\mathfrak{s l}_{N}$-módulo, porém se a identidade age por $\alpha \in \mathbb{C}$ em $W$, em $W^{*}$ ela agirá por $N-\alpha$. Como consequência da existência desta forma é provado o seguinte lema:

Lema 5.3.2. A condição $(C)$ vale para $L(W, \gamma, h)$ se, e somente se, a condição $(G)$ vale para $L\left(W^{*}, \gamma, h\right)$.

Com este lemma a questão se reduz a ver se os módulos $L(W, \gamma, h)$ e $L\left(W^{*}, \gamma, h\right)$ satisfazem a condição (C). Em caso afirmativo ambos serão irredutíveis.

Lembrando que $a(z)$ _ é a parte de potências negativas de $z$ da série $a(z)$ (seção 4.1). Seja $g \in V$ um vetor crítico, temos que $\left(z d_{a}(r, z)\right)_{-} g=0$ expandindo a ação da série temos

$$
\begin{gathered}
q^{r}\left(\exp \left(\sum_{p=1}^{N} r^{p} \sum_{j=1}^{\infty} u_{p j} z^{j}\right)\left(\sum_{i=1}^{\infty} i v_{a i} z^{i}+q_{a} \frac{\partial}{\partial q_{a}}+\sum_{p=1}^{N} r_{p} \sum_{k \in \mathbb{Z}} E_{(k)}^{p a} z^{-k}\right) \times\right. \\
\left.\times \exp \left(-\sum_{p=1}^{N} r_{p} \sum_{j=1}^{\infty} \frac{z^{-j}}{j} \frac{\partial}{\partial v_{p j}}\right)\right)_{-} g=0
\end{gathered}
$$

Inicialmente, observe que $\mathcal{D}^{+}$contem elementos da forma $t_{0}^{j} d_{p}$ com $p \in\{1, \ldots, N\}$ e $j \geq 1$, que agem por $\partial / \partial u_{p j}$. Para obter sua ação no módulo devemos comparar os elementos de mesma 
potência nas séries do teorema 5 .1.3. Devido a esta ação podemos projetar no operador $\left(z d_{a}(r, z)\right)_{-}$ no espaço

$$
q^{\gamma} \mathbb{C}\left[q_{1}^{ \pm 1}, \ldots, q_{N}^{ \pm 1}\right] \otimes \mathbb{C}\left[\left.v_{p j}\right|_{j=1,2, \ldots} ^{p=1, \ldots, N}\right] \otimes L_{g_{N}}(W) \otimes L_{\mathrm{Vir}}(h),
$$

pois como $g$ não pode depender de variáveis $u_{p j}$ ele pertence a este espaço. Assim, obtemos

$$
P_{a}(r, z)_{-} g=0
$$

onde

$$
P_{a}(r, z)=\left(\sum_{i=1}^{\infty} i v_{a i} z^{i}+q_{a} \frac{\partial}{\partial q_{a}}+\sum_{p=1}^{N} r_{p} \sum_{k \in \mathbb{Z}} E_{(k)}^{p a} z^{-k}\right) \exp \left(-\sum_{p=1}^{N} r_{p} \sum_{j=1}^{\infty} \frac{z^{-j}}{j} \frac{\partial}{\partial v_{p j}}\right) .
$$

Observe que também retiramos a multiplicação por $q^{r}$, já que este elemento é invertível.

Podemos expandir o operador $P_{a}(r, z)$ em uma serie formal nas variáveis $R=\left(r_{1}, \ldots, r_{N}\right)$ :

$$
P_{a}(r, z)=\sum_{s \in \mathbb{Z}_{+}^{N}} r^{s} P_{a s}(z)
$$

onde $\mathbb{Z}_{+}^{N}$ é o conjunto das $N$-uplas com entradas inteiras e positivas.

Para todo $j \in \mathbb{Z}$ e todo vetor $g^{\prime}$, existe apenas uma quantidade finita de $s \in \mathbb{Z}_{+}^{N}$ tal que o coeficiente de $z^{j}$ em $P_{a s}(z) g^{\prime}$ seja não nulo. Assim, o coeficiente de $z^{j}$ em $\sum_{s \in \mathbb{Z}_{+}^{N}} r^{s} P_{a s}(z) g$ é um polinômio em $r$. Como para cada $j<0$ estes polinômios se anulam para todo $r \in \mathbb{Z}^{N}$, podemos concluir que para todo $s \in \mathbb{Z}_{+}^{N}, a \in\{1, \ldots, N\}$,

$$
P_{a s}(z)_{-} g=0 \text {. }
$$

Para o caso particular $s_{p} \in \mathbb{Z}_{+}^{N}$ com entrada 1 na coordenada $p$ e 0 nas demais, obtemos

$$
\sum_{k=1}^{\infty} z^{-k} E_{(k)}^{p a} g=\left(\sum_{k=1}^{\infty}\left(\sum_{i=1}^{k-1} i v_{a i} z^{i}+q_{a} \frac{\partial}{\partial q_{a}}\right) \frac{z^{-k}}{k} \frac{\partial}{\partial v_{p k}}\right) g .
$$

Assim, obtemos

$$
P_{a}^{\prime}(r, z)_{-} g=0
$$

onde

$$
\begin{gathered}
P_{a}^{\prime}(r, z)_{-}=\left(\sum_{i=1}^{\infty} i v_{a i} z^{i}+q_{a} \frac{\partial}{\partial q_{a}}+\sum_{p=1}^{N} r_{p} \sum_{k=0}^{\infty} E_{(-k)}^{p a} z^{-k}\right) \exp \left(-\sum_{p=1}^{N} r_{p} \sum_{j=1}^{\infty} \frac{z^{-j}}{j} \frac{\partial}{\partial v_{p j}}\right)+ \\
+\left(\sum_{p=1}^{N} r_{p} \sum_{k=1}^{\infty}\left(\sum_{i=1}^{k-1} i v_{a i} z^{i}+q_{a} \frac{\partial}{\partial q_{a}}\right) \frac{z^{-k}}{k} \frac{\partial}{\partial v_{p k}}\right) \exp \left(-\sum_{p=1}^{N} r_{p} \sum_{j=1}^{\infty} \frac{z^{-j}}{j} \frac{\partial}{\partial v_{p j}}\right) .
\end{gathered}
$$

Podemos definir uma $\mathbb{Z}$ graduação em cada um dos fatores do produto tensorial $\mathbb{C}\left[\left.v_{p j}\right|_{j=1,2, \ldots} ^{p=1, \ldots, N}\right] \otimes$ $L_{\mathfrak{g l}}(W) \otimes L_{\mathrm{Vir}}(h)$ da seguinte maneira

$$
\begin{gathered}
\operatorname{deg}\left(v_{p j}\right)=\operatorname{deg}\left(E_{(-j)}^{a b}\right)=\operatorname{deg}\left(L_{(-j)}\right)=j, \\
\operatorname{deg}(W)=\operatorname{deg}\left(v_{h}\right)=0,
\end{gathered}
$$

onde deg representa o grau da graduação (degree em inglês).

Fixando uma base homogênea com relação às graduações, digamos $\left\{y_{i}^{\prime}\right\},\left\{y_{j}^{\prime \prime}\right\},\left\{y_{k}^{\prime \prime \prime}\right\}$ para os 
espaços $\mathbb{C}\left[\left.v_{p j}\right|_{j=1,2, \ldots} ^{p=1, \ldots, N}\right], L_{\mathfrak{g l}}(W)$ e $L_{\bigvee i r}(h)$, respectivamente, podemos escrever um vetor $g$ como

$$
g=\sum_{i j k} \alpha_{i j k} q^{\mu} y_{i}^{\prime} \otimes y_{j}^{\prime \prime} \otimes y_{k}^{\prime \prime \prime}
$$

Lema 5.3.3. [9.3 de [3]] Seja g um vetor crítico não nulo. Ențão nesta decomposição apresentada existem $y_{j}^{\prime \prime} \in W$ e $y_{k}^{\prime \prime \prime} \in \mathbb{C} v_{h}$ tais que $\alpha_{i j k} \neq 0$ para algum $i$.

Projetando a equação $P_{n}^{\prime}(r, z)_{-} g=0$, no espaço

$$
q^{\mu} \mathbb{C}\left[\left.v_{p j}\right|_{j=1,2, \ldots} ^{p=1, \ldots, N}\right] \otimes \bar{W} \otimes v_{h},
$$

obtemos

$$
P_{a}^{\prime \prime}(r, z)-\bar{g}=0,
$$

onde $\bar{g}$ é a projeção de $g$ neste espaço e

$$
\begin{gathered}
P_{a}^{\prime \prime}(r, z)_{-}=\left(\sum_{i=1}^{\infty} i v_{a i} z^{i}+q_{a} \frac{\partial}{\partial q_{a}}+\sum_{p=1}^{N} r_{p} \sum_{k=0}^{\infty} E_{(0)}^{p a}\right) \exp \left(-\sum_{p=1}^{N} r_{p} \sum_{j=1}^{\infty} \frac{z^{-j}}{j} \frac{\partial}{\partial v_{p j}}\right)+ \\
+\left(\sum_{p=1}^{N} r_{p} \sum_{k=1}^{\infty}\left(\sum_{i=1}^{k-1} i v_{a i} z^{i}+q_{a} \frac{\partial}{\partial q_{a}}\right) \frac{z^{-k}}{k} \frac{\partial}{\partial v_{p k}}\right) \exp \left(-\sum_{p=1}^{N} r_{p} \sum_{j=1}^{\infty} \frac{z^{-j}}{j} \frac{\partial}{\partial v_{p j}}\right)
\end{gathered}
$$

Novamente, tomamos a série formal nas variáveis $r, P_{a}^{i \prime}(r, z)=\sum_{s \in \mathbb{Z}_{+}^{N}} r^{s} P_{a s}^{i f}(z)$ e obtemos

$$
P_{a s}^{\prime \prime}(z)-\bar{g}=0
$$

para todo $s \in \mathbb{Z}_{+}^{N}$.

Existe ainda uma outra graduação. Considere a seguinte $\mathbb{Z}^{N}$-graduação

$$
\operatorname{len}_{a}\left(v_{p j}\right)=\delta_{a p},
$$

onde len vem de comprimento (lenght em inglês), o comprimento total de um monômio $y \in \mathbb{C}\left[v_{p j}\right]$ é dado por

$$
\operatorname{len}(y)=\sum_{a=1}^{N} \operatorname{len}_{a}(y) .
$$

Os operadores $P_{a s}^{\prime \prime}(z)$ contêm duas componentes homogêneas com relação ao comprimento. Uma que reduz o comprimento por $s_{1}+\ldots+s_{N}-1$, denotado por $Q_{a s}(z)$, e outra, que contem os termos contendo $q_{a} \frac{\partial}{\partial q_{a}}$ e reduz o comprimento por $s_{1}+\ldots+s_{N}$.

Seja $f$ a componente de maior comprimento de $\bar{g}$, digamos len $(f)=l$. Então, a componente de comprimento $l+1-s_{1}-\ldots-s_{N}$ em $P_{a s}^{\prime \prime}(z)_{-} \bar{g}$ é $Q_{a s}(z)_{-} f$. Logo

$$
Q_{a s}(z)_{-} f=0 \text {. }
$$

Os lemas 9.5 e 9.6 de [3] garantem que soluções homogêneas desta última equação têm comprimento 1. Suponha que $f$ tem grau $m$, cntão $f$ podc scr cscrito como

$$
f=\sum_{p=1}^{N} v_{p m} \otimes w_{p}, \quad w_{p} \in W .
$$


Substituindo na equação obtemos

$$
\sum_{p=1}^{N} \sum_{b=1}^{N} r_{p} r_{b}\left(E^{p a}+\delta_{p a}(m-1)\right) w_{b}=0 .
$$

Se considerarmos uma nova ação $\rho^{\prime}$ de $\mathfrak{g l}_{N}$ em $W$ :

$$
\rho^{\prime}\left(E^{p a}\right) w=E^{p a}+m \delta_{p a} w,
$$

então a última equação é equivalente ao sistema

$$
\rho^{\prime}\left(E^{c a}\right) w_{b}+\rho^{\prime}\left(E^{b a}\right) w_{c}=\delta_{c a} w_{b}+\delta b a w_{c},
$$

onde $a, b, c \in\{1, \ldots, N\}$.

Seja $\mathcal{P}$ o conjunto de toas $N$-uplas soluções deste sistema. O lema 9.7 de [3] nos diz que

$$
\widetilde{\mathcal{P}}=\left\{\bigoplus_{r \in \mathbb{Z}^{N}} q^{r} \otimes\left(r_{1} w_{1}+\ldots+r_{N} w_{N}\right) \mid\left(w_{1}, \ldots, w_{N}\right) \in \mathcal{P}\right\}
$$

é um Vect $\left(\mathbb{T}^{N}\right)$-submódulo de $T(W)$. Como corolário temos que se $L(W, \gamma, h)$ contém um vetor crítico não trivial então $W$ é um módulo trivial para $s l_{N}$ com a identidade agindo por $\alpha=N-m N$, $m=1,2, \ldots$, ou o peso máximo de $W$ é um peso fundamental $\omega_{k}, k=1, \ldots, k-1$, com a identidade agindo por $\alpha=k-m N$, ou $W$ é um módulo de dimensão 1 com a identidade agindo por $\alpha=N-m N$.

Por fim, o lema 9.9 mostra que se $L(W, \gamma, h)$ contem vetor crítico não trivial então $h=0$. A ideia é praticamente a mesma empregada para mostrar o formato de $W$ só que desta fez considere a equação:

$$
\left(z^{2} d_{0}(r, z)\right)_{-} g=0
$$

\subsection{Chiral de de Rham}

O chiral de de Rham foi introduzido em [15]. No caso de um toro o espaço desse chiral é o produto de duas superálgebras de vértice

$$
V_{\mathrm{Hyp}}^{+} \otimes V_{\mathbb{Z}^{N}}
$$

Considere os seguintes campos

$$
E^{a b}(z)=: \phi^{a}(z) \psi^{b}(z):
$$

eles geram uma subálgebra de $V_{\mathbb{Z}^{N}}$ que é uma álgebra de vértice irredutível de $\widehat{\mathfrak{g l}}_{N}$ com carga central 1 .

Para $V_{\mathbb{Z}^{N}}$ apresentamos duas $\mathbb{Z}$-graduações. A graduação fermiônica definida como:

$$
\operatorname{deg}_{f e r}\left(\phi_{(j)}^{p}\right)=1, \quad \operatorname{deg}_{f e r}\left(\psi_{(j)}^{p}\right)=-1, \quad \operatorname{deg}_{f e r}(K)=\operatorname{deg}_{f e r}(\mathbb{1})=0,
$$

e a bosônica:

$$
\operatorname{deg}_{b o s}\left(\phi_{(j)}^{p}\right)=-j-1, \quad \operatorname{deg}_{b o s}\left(\psi_{(j)}^{p}\right)=-j, \quad \operatorname{deg}_{b o s}(K)=\operatorname{deg}_{b o s}(\mathbb{1})=0 .
$$

Seja $V_{\mathbb{Z}^{N}}^{k}$ o subespaço de elementos de grau fermiônico $k$. Temos a decomposição

$$
V_{\mathbb{Z}^{N}}=\bigoplus_{k \in \mathbb{Z}} V_{\mathbb{Z}^{N}}^{k}
$$


Cada subespaço $V_{\mathbb{Z}^{N}}^{k}$ é um $\widehat{\mathfrak{g l}}_{N^{N}}$-módulo graduado pela graduação bosônica. Sua estrutura é descrita em [13], para nós será importante o seguinte teorema:

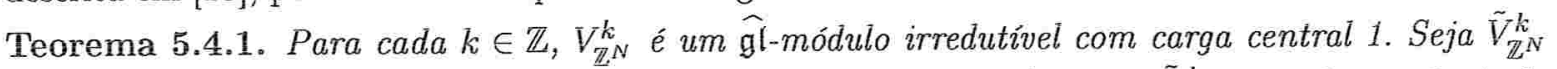
a componente de $V_{\mathbb{Z}^{N}}^{k}$ de menor grau bosônico. Se $k \equiv 0(\bmod N)$ então $\tilde{V}_{\mathbb{Z}^{N}}^{k}$ tem dimensão 1 . Se $k=k^{\prime}(\bmod N)$ com $1 \leq k^{\prime}<N$ então, como módulo sobre $\mathfrak{s l}_{N}, \tilde{V}_{\mathbb{Z}^{N}}^{k}$ tem como peso máximo o peso fundamental $\omega_{k^{\prime}}$. E a matriz identidade de $\mathfrak{g l}_{N}$ age como $k$ Id.

Em conjunto com o teorema 5.1.3 temos o seguinte corolário:

Corolário 5.4.2. O espaço

$$
M_{\mathrm{Hyp}} \otimes V_{\mathbb{Z}^{N}}^{k}
$$

para todo $k \in \mathbb{Z}$, tem estrutura de módulo sobre a álgebra de Lie Vect $\left(\mathbb{T}^{N+1}\right)$.

Restringindo a ação da álgebra Vect $\left(\mathbb{T}^{N+1}\right)$, dada pelo teorema 5.1 .3 , no espaço $M_{\mathrm{Hyp}} \otimes V_{\mathbb{Z}^{N}}^{k}$ obtemos:

$$
d_{a}(r, z) \rightarrow Y\left(d_{a}(r), z\right), d_{0}(r, z) \rightarrow Y\left(d_{0}(r), z\right),
$$

onde

$$
\begin{gathered}
d_{a}(r)=v_{(-1)}^{a} q^{r}+\sum_{p=1}^{N} r_{p} \phi_{(-1)}^{p} \psi_{(-1)}^{a} q^{r} \\
d_{0}(r)=-\left(\sum_{p=1}^{N} u_{(-1)}^{p} v_{(-1)}^{p} q^{r}+\sum_{p=1}^{N} \phi_{(-2)}^{p} \psi_{(-1)}^{p} q^{r}+\sum_{a, b=1}^{N} r_{a} u_{(-1)}^{b} \phi_{(-1)}^{a} \psi_{(-1)}^{b} q^{r}\right)
\end{gathered}
$$

Seja

$$
Q=\sum_{p=1}^{N} v_{(-1)}^{p} \phi_{(-1)}^{p} \mathbb{1}
$$

e definamos $\mathrm{d}=Q_{(}(0)$, i. e., o coeficiente de $z^{-1}$ em $Y(Q, z)=\sum_{p=1}^{N}: v^{p}(z) \phi^{p}(z):$. Este operador d será a diferencial do chiral de de Rham:

$$
\cdots \stackrel{\text { d }}{\rightarrow} M_{\text {Hyp }} \otimes V_{\mathbb{Z}^{N}}^{k} \stackrel{\text { d }}{\rightarrow} M_{\text {Hyp }} \otimes V_{\mathbb{Z}^{N}}^{k+1} \stackrel{\text { d }}{\rightarrow} \ldots
$$

Para ver que $\mathrm{d} \circ \mathrm{d}=0$ observe que

$$
\left[Y\left(Q, z_{1}\right), Y\left(Q, z_{2}\right)\right]=\sum_{n>0} \frac{1}{n !} Y\left(Q_{(n)} Q, z_{2}\right)\left[z_{1}^{-1}\left(\frac{\partial}{\partial z_{2}}\right)^{n} \delta\left(\frac{z_{2}}{z_{1}}\right)\right]=0 .
$$

Para o próximo teorema precisaremos do seguinte lema.

Lema 5.4.3. Seja $V$ uma superálgebra de vértice e sejam $a, b \in V$, tais que $a_{(0)} b=0$. Então

$$
\left[a_{(0)}, Y(b, z)\right]=0 .
$$

Demonstração. Como $a_{(0)} b=0$ a fórmula do comutador nos dá

$$
\left[Y\left(a, z_{1}\right), Y\left(b, z_{2}\right)\right]=\sum_{n>1} \frac{1}{n !} Y\left(a_{(n)} b, z_{2}\right)\left[z_{1}^{-1}\left(\frac{\partial}{\partial z_{2}}\right)^{n} \delta\left(\frac{z_{2}}{z_{1}}\right)\right]
$$

observe que o lado direito não tem termos de $z^{-1} \operatorname{logo}\left[a_{(0)}, Y(b, z)\right]=0$.

Teorema 5.4.4. A aplicação

$$
\mathrm{d}: M_{\mathrm{Hyp}} \otimes V_{\mathbb{Z}^{N}}^{k} \rightarrow M_{\mathrm{Hyp}} \otimes V_{\mathbb{Z}^{N}}^{k+1},
$$


é um homomorfismo de $\operatorname{Vect}\left(\mathbb{T}^{N+1}\right)$-módulos.

Demonstração. Para provar este teorema devemos mostrar que

$$
\begin{aligned}
& {\left[\mathrm{d}, d_{a}(r, z)\right]=0,} \\
& {\left[\mathrm{~d}, d_{0}(r, z)\right]=0 .}
\end{aligned}
$$

Segue do lema que basta provar

$$
Q_{(0)} d_{a}(r)=0 \text { e } Q_{(0)} d_{0}(r)=0 .
$$

Da definição $Y(Q, z)=\sum_{p=1}^{N}: v^{p}(z) \phi^{p}(z):$ temos:

$$
Q_{(0)}=\sum_{i=1}^{N}\left(\sum_{j=0}^{\infty} \phi_{(-j-1)}^{i} v_{(j)}^{i}+\sum_{j=1}^{\infty} v_{(-j)}^{i} \phi_{(j-1)}^{i}\right) .
$$

Observe que $v_{(j)}^{i} d_{a}(r)=0$ e $\phi_{(j)}^{i} d_{a}(r)=0$ para $j \geq 1$, logo

$$
\begin{gathered}
Q_{(0)} d_{a}(r)=\sum_{i=1}^{N}\left(\phi_{(-1)}^{i} v_{(0)}^{i}+v_{(-1)}^{i} \phi_{(0)}^{i}\right) d_{a}(r)= \\
=\sum_{I=1}^{N} r_{i} \phi_{(-1)}^{i} v_{(-1)}^{a} q^{r}-\sum_{i=1}^{N} \sum_{p=1}^{N} r_{p} v_{(-1)}^{i} \phi_{(-1)}^{p} \phi_{(0)}^{i} \psi_{(-1)}^{a} q^{r}= \\
=\sum_{i=1}^{N} r_{i} \phi_{(-1)}^{i} v_{(-1)}^{a} q^{r}-\sum_{p=1}^{N} r_{p} v_{(-1)}^{a} \phi_{(-1)}^{p} q^{r}=0 .
\end{gathered}
$$

Para mostrar que $Q_{(0)} d_{0}(r)=0$, inicialmente, observe que $v_{(j)}^{i} d_{0}(r)=0$ para $j \geq 2$ e $\phi_{(j)}^{i} d_{0}(r)=$ 0 para $j \geq 1$, com isto obtemos

$$
-Q_{(0)} d_{0}(r)=\sum_{i=1}^{N}\left(\phi_{(-1)}^{i} v_{(0)}^{i}+\phi_{(-2)}^{i} v_{(1)}^{i}+v_{(-1)}^{i} \phi_{(0)}^{i}\right)\left(-d_{0}(r)\right) .
$$

Vamos dividir esta soma em três partes. A primeira parte nos dá:

$$
\begin{gathered}
\left(\sum_{i=1}^{N} \phi_{(-1)}^{i} v_{(0)}^{i}\right)\left(-d_{0}(r)\right)= \\
=\sum_{i=1}^{N} \sum_{p=1}^{N} \phi_{(-1)}^{i} v_{(0)}^{i} u_{(-1)}^{p} v_{(-1)}^{p} q^{r}+\sum_{i=1}^{N} \sum_{p=1}^{N} \phi_{(-1)}^{i} v_{(0)}^{i} \phi_{(-2)}^{p} \psi_{(-1)}^{p} q^{r}+ \\
+\sum_{i=1}^{N} \sum_{a, b=1}^{N} r_{a} \phi_{(-1)}^{i} v_{(0)}^{i} u_{(-1)}^{b} \phi_{(-1)}^{a} \psi_{(-1)}^{b} q^{r}= \\
=\sum_{i=1}^{N} \sum_{p=1}^{N} r_{i} \phi_{(-1)}^{i} u_{(-1)}^{p} v_{(-1)}^{p} q^{r}+\sum_{i=1}^{N} \sum_{p=1}^{N} r_{i} \phi_{(-1)}^{i} \phi_{(-2)}^{p} \psi_{(-1)}^{p} q^{r}+ \\
\sum_{i=1}^{N} \sum_{a, b=1}^{N} r_{a} r_{i} u_{(-1)}^{b} \phi_{(-1)}^{i} \phi_{(-1)}^{a} \psi_{(-1)}^{b} q^{r} .
\end{gathered}
$$

Observe que esta última soma é anti-simétrica em $\{a, i\}$, logo vale 0 . A segunda parte da soma: 


$$
\begin{gathered}
\left(\sum_{i=1}^{N} \dot{\phi}_{(-2)}^{i} v_{(1)}^{i}\right)\left(-d_{0}(r)\right)= \\
\sum_{i=1}^{N} \sum_{p=1}^{N} \phi_{(-2)}^{i} v_{(1)}^{i} u_{(-1)}^{p} v_{(-1)}^{p} q^{r}+\sum_{i=1}^{N} \sum_{p=1}^{N} \phi_{(-2)}^{i} v_{(1)}^{i} \phi_{(-2)}^{p} \psi_{(-1)}^{p} q^{r}+ \\
+\sum_{i=1}^{N} \sum_{a, b=1}^{N} r_{a} \phi_{(-2)}^{i} v_{(1)}^{i} u_{(-1)}^{b} \phi_{(-1)}^{a} \psi_{(-1)}^{b} q^{r}= \\
=\sum_{p=1}^{N} \phi_{(-2)}^{p} v_{(-1)}^{p} q^{r}+\sum_{i=1}^{N} \sum_{a=1}^{N} r_{a} \phi_{(-2)}^{i} \phi_{(-1)}^{a} \psi_{(-1)}^{i} q^{r}
\end{gathered}
$$

$\mathrm{E}$, finalmente, a terceira parte:

$$
\begin{gathered}
\left(\sum_{i=1}^{N} v_{(-1)}^{i} \phi_{(0)}^{i}\right)\left(-d_{0}(r)\right)= \\
=\sum_{i=1}^{N} \sum_{p=1}^{N} v_{(-1)}^{i} \phi_{(0)}^{i} u_{(-1)}^{p} v_{(-1)}^{p} q^{r}+\sum_{i=1}^{N} \sum_{p=1}^{N} v_{(-1)}^{i} \phi_{(0)}^{i} \phi_{(-2)}^{p} \psi_{(-1)}^{p} q^{r}+ \\
+\sum_{i=1}^{N} \sum_{a, b=1}^{N} r_{a} v_{(-1)}^{i} \phi_{(0)}^{i} u_{(-1)}^{b} \phi_{(-1)}^{a} \psi_{(-1)}^{b} q^{r}= \\
=-\sum_{p=1}^{N} v_{(-1)}^{p} \phi_{(-2)}^{p} q^{r}-\sum_{i=1}^{N} \sum_{a=1}^{N} r_{a} v_{(-1)}^{i} u_{(-1)}^{i} \phi_{(-1)}^{a} q^{r} .
\end{gathered}
$$

Combinando estas três partes obtemos $Q_{(0)} d_{0}(r)=0$.

Exemplo 4. Como exemplo tome o caso $N=2$. Apresentamos o diagrama do chiral de de Rham, o grau fermiônico varia na direção horizontal e o bosônico na direção vertical.

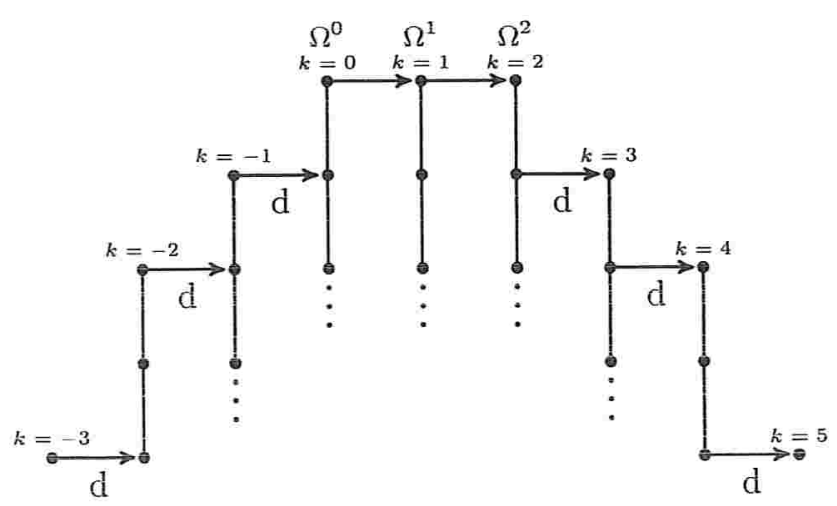

O topo dos módulos $M_{\mathrm{Hyp}}(\gamma) \otimes V_{\mathbb{Z}^{N}}^{k} \operatorname{com} k \in\{0, \ldots, N\}$ são os espaços $q^{\gamma} \Omega^{k}\left(\mathbb{T}^{N}\right)$ da cadeia do complexo de de Rham clássico. Áqueles que tiverem submódulos não triviais para $\operatorname{Vect}\left(\mathbb{T}^{N+1}\right)$ gerarão submódulos não triviais em $M_{\mathrm{Hyp}}(\gamma) \otimes V_{\mathbb{Z}^{N}}^{k}$.

Além disso, temos que para $k \leq 0$ os módulos $M_{\mathrm{Hyp}}(\gamma) \otimes V_{\mathbb{Z}^{N}}^{k}$ têm vetores críticos não triviais e para $k \geq N$ os módulos $M_{\text {Hyp }}(\gamma) \otimes V_{\mathbb{Z}^{N}}^{k}$ não são gerados pelos seus topos.

Com isso, concluímos que os módulos para os quais o teorema 5.3 .1 junto com o lema 5.3 .2 não garantem a irredutibilidade são, na verdade, redutíveis. 


\section{Apêndice A}

\section{Álgebras de Lie}

Neste apêndice apresentamos algumas definições e propriedades básicas de álgebras de Lie que são utilizadas na dissertação. Para isso seguimos principalmente [9] e [17]. Para as álgebras afim seguimos [10].

As álgebras de Lie surgiram com Sophus Lie na década de 1870, dentro de seu programa de estender, às equações diferenciais, a teoria de Galois para equações algébricas. As álgebras de Lie aparecem como objetos infinitesimais associados aos grupos de transformações agindo sobre um certo espaço, em que um dos principais problemas era sua classificação. Em 1884 Wilhelm Killing dividiu o problema em dois: o de classificar os objetos abstratos que correspondem as álgebra de Lie e posteriormente analisar as ações dos grupos correspondentes. O termo álgebra de Lie foi popularizado a partir da década de 1920 com Hermann Weyl, em substituição ao grupo infinitesimal que se utilizava desde os tempos de Lie.

A seguir temos a definição de uma álgebra de Lie.

Definição A.0.5. Uma álgebra de Lie consiste de espaço vetorial $\mathfrak{g}$ sobre um corpo $k$, munido do produto colchete de Lie, que é definido como

$$
[,]: \mathfrak{g} \times \mathfrak{g} \rightarrow \mathfrak{g},
$$

que respeita as seguintes propriedades

1. é bilinear;

2. é anti-simétrico, i. e., $\forall x, y \in \mathfrak{g},[x, x]=0$. Isto implica que o colchete é anti comutativo $[x, y]=-[y, x]$

3. satisfaz a identidade de Jacobi, $\forall x, y, z \in \mathfrak{g}$,

$$
[x,[y, z]]=[[x, y], z]+[y,[x, z]] .
$$

Exemplo 5. Dado um espaço vetorial $V$, podemos definir o colchete de Lie identicamente nulo $[v, w]=0, \forall v, w \in V$, neste caso diremos que $V$ é abeliano. Observe que toda álgebra de dimensão 1 é abeliana.

Exemplo 6. Dada uma álgebra associativa $A$, definimos $A^{-}$como sendo a álgebra de Lie com o colchete dado pelo comutador, que é definido como

$$
[a, b]=a b-b a,
$$

onde $a, b \in A$.

Exemplo 7. Dado uma álgebra $A$, dizemos que um endomorfismo $d$ do espaço vetorial $A$ é uma derivada se, para todo $a, b \in A$,

$$
d(a \cdot b)=d(a) \cdot b+a \cdot d(b) .
$$


O conjunto de todas as derivações de $A$ é denotado por $\operatorname{Der}(A)$, além disso, observe que para todos $d, d^{\prime} \in \operatorname{Der}(A)$ tem-se que $\left[d, d^{\prime}\right]=d \circ d^{\prime}-d^{\prime} \circ d$ (onde o denota a composição) está em $\operatorname{Der}(A)$ e define um estrutura de álgebra de Lie. Em particular, tomemos $A=\mathbb{C}\left[z^{ \pm 1}\right]$, para definirmos $d \in$ $\operatorname{Der}(A)$ apenas precisamos definir $d(z)$, com efeito, para $n>0$

$$
d\left(z^{n}\right)=d\left(z^{n-1}\right) z+z^{n-1} d(z),
$$

observe ainda que

$$
d(1)=d(1)+d(1)=2 d(1)=0 \Rightarrow d\left(z z^{-1}\right)=0 \Rightarrow d\left(z^{-1}\right)=-z^{-2} d(z),
$$

e por fim

$$
d\left(z^{-n}\right)=d\left(z^{-n+1}\right) z^{-1}+z^{-n+1} d\left(z^{-1}\right) .
$$

Definimos

$$
L_{n}:=-z^{n+1} \frac{\partial}{\partial z}
$$

claramente $L_{n} \in \operatorname{Der}(A)$ e como $L_{n}(z)$ é uma base de $A$, então eles são uma base de $\operatorname{Der}(A)$.

Exemplo 8. Seja $\mathfrak{g}$ uma álgebra de Lie, e sejam $x, y \in \mathfrak{g}$, considere o operador $\operatorname{ad}(x): \mathfrak{g} \rightarrow \mathfrak{g}$ definido como $\operatorname{ad}(x)(y)=[x, y]$. Observe que pela identidade de Jacobi tal operador é uma derivação para todo $x \in \mathfrak{g}$. Esta é uma maneira de memorizar tal identidade. Vamos chamar de adg o conjunto de todos operadores da forma ad( $(x)$ para algum $x \in \mathfrak{g}$.

Exemplo 9. Um grupo de Lie é uma variedade diferenciável com uma estrutura de grupo tal que a operação do grupo e a inversão sejam $\mathcal{C}^{\infty}$, isto é, infinitamente diferenciáveis, onde a inversão se entende por uma aplicação que leva um elemento no seu elemento simétrico. Uma propriedade dos grupos de Lie é que o espaço tangente na identidade é uma álgebra de Lie. Dois exemplos importantes são $g l_{n}(\tilde{k})=\left\{\hat{A} \in M_{n}(\mathbf{k}) \mid \operatorname{det}(A) \neq 0\right\}$ e $s l_{n}(\mathbf{k})=\left\{A \in M_{n}(\mathbf{k}) \mid \operatorname{det}(A)=1\right\}$, onde $\mathrm{k}$ é $\mathbb{C}$ ou $\mathbb{R}$, estes dois conjuntos admitem uma estrutura de variedade e com o produto usual de matrizes, eles possuem estrutura de grupo. Suas álgebras de Lie são $\mathfrak{g l}_{n}(\mathbf{k})=M_{n}(\mathbf{k})$ e $\mathfrak{s l}_{n}(\mathbf{k})=\left\{A \in M_{n}(\mathbf{k}) \mid \operatorname{Tr}(A)=0\right\}$, respectivamente. Em ambos os casos o colchete é dado pelo comutador. Uma prova deste fato, para $\mathbf{k}=\mathbb{R}$, pode ser vista em [8].

Algumas definições importantes:

Definição A.0.6. Seja g uma álgebra de Lie. Uma subálgebra de Lie de g é um subespaço vetorial $\mathfrak{h}$ de $\mathfrak{g}$ que é fechado pelo colchete, isto é, $[\mathfrak{h}, \mathfrak{h}] \subset \mathfrak{h}$, se além disso tivermos $[\mathfrak{g}, \mathfrak{h}] \subset \mathfrak{h}$, diremos que $\mathfrak{h}$ é um ideal de g. Uma álgebra g de dimensão maior que um tal que só contenha como ideais g e 0 é dita simples.

Definição A.0.7. A série derivada da álgebra de Lie g é definida, por recursão, como

$$
\begin{gathered}
\mathfrak{g}^{(0)}=\mathfrak{g}, \\
\mathfrak{g}^{\prime}=[\mathfrak{g}, \mathfrak{g}], \\
\mathfrak{g}^{(k)}=\left[\mathfrak{g}^{(k-1)}, \mathfrak{g}^{(k-1)}\right] .
\end{gathered}
$$

Uma álgebra é dita solúvel se sua série derivada se anula em algum momento e semi-simples se não possuir ideais solúveis além do 0 . É possível mostrar que uma álgebra é semi-simples se, e somente se, for soma direta de álgebras simples.

Analogamente, a série central descendente da álgebra de Lie g é definida, por recursão, como

$$
\begin{gathered}
\mathfrak{g}^{1}=\mathfrak{g}, \\
\mathfrak{g}^{2}=[\mathfrak{g}, \mathfrak{g}], \\
\mathfrak{g}^{k}=\left[\mathfrak{g}, \mathfrak{g}^{k-1}\right] .
\end{gathered}
$$

Uma álgebra é dita nilpotente se sua série central descendente se anula em algum momento. 
Do exemplo 8 temos que adg $\subset \operatorname{Der}(g)$, um fato, cuja prova pode ser vista em [9] teorema ว̆.3, é que temos a igualdade para álgebras semi-simples. Isto é, para toda derivação existe $x \in \mathfrak{g}$ tal que esta derivação seja $\operatorname{ad}(x)$.

Ainda, do exemplo 8, para álgebras de dimensão finita podemos definir a seguinte forma $\kappa(x, y)=$ $\operatorname{Tr}(\operatorname{ad}(x) \operatorname{ad}(y))$, onde $x, y$ são elementos da álgebra. Esta forma é chamada de forma de Killing. $\mathbf{E}$ fácil ver que a forma de Killing é uma forma bilinear e simétrica.

A partir de agora precisaremos que $\mathfrak{g}$ seja de dimensão finita. Seja $x \in \mathfrak{g}$ e considere a decomposição de $\operatorname{Jordan} \operatorname{de} \operatorname{ad}(x)=\operatorname{ad}(x)_{s}+\operatorname{ad}(x)_{n}, \operatorname{com} \operatorname{ad}(x)_{s}$ a parte semi-simples e ad $(x)_{n}$ a parte nilpotente, ambas são unicamente definidas e, além disso, são derivações. Assim, existem únicos $x_{s}$ e $x_{n} \in \mathfrak{g}$, tais que $\operatorname{ad}(x)=\operatorname{ad}\left(x_{s}\right)+\operatorname{ad}\left(x_{n}\right)$, estes elementos são chamados parte semi-simples e nilpotente de $x$, respectivamente.

Para o resultado que se segue precisaremos que o corpo seja algebricamente fechado. Seja $\mathfrak{h} \neq\{0\}$ uma subálgebra de $\mathfrak{g}$ formada por elementos semi-simples, isto é, sua parte nilpotente é nula. Seja $x \neq 0 \in \mathfrak{h}$, como $x$ é semi-simples e o corpo é fechado, ad(x) é diagonalizável, seja $y \neq 0 \in \mathfrak{h}$ autovetor de $\operatorname{ad}(x)$. Temos $[x, y]=a y$, com $a \in k$, logo ad $(y)(x)=-a y$, aplicando novamente $\operatorname{ad}(y)$, temos que ad $(y)(x)$ é autovetor de autovalor 0 para ad $(y)$, o que implica que $x$ é autovetor de autovalor 0 para $\operatorname{ad}(y)$, isto é, $[x, y]=0$. Como $x$ foi qualquer e $y$ foi um autovetor qualquer temos que $\mathfrak{h}$ é abeliana. Tal subálgebra é dita subálgebra toral, uma subálgebra toral não contida propriamente em nenhuma outra subálgebra toral é dita subálgebra toral maximal.

Seja $\mathfrak{h}$ uma subálgebra toral maximal, como todo elemento de adh é diagonalizável e como $\mathfrak{h}$ é abeliana, temos que os elementos de adh são mutualmente diagonalizáveis. Assim, para todo $\lambda \in \mathfrak{h}^{*}$, podemos definir $\mathfrak{g}_{\lambda}:=\{x \in \mathfrak{g} \mid \forall h \in \mathfrak{h},[h, x]=\lambda(h) x\}$ e teremos

$$
\mathfrak{g}=C(\mathfrak{h}) \oplus_{\lambda \in \mathfrak{h}^{*}} g_{\lambda}
$$

onde $C(\mathfrak{h})$ é o centralizador de $\mathfrak{h}$. A proposição 8.2 de [9] mostra que o centralizador de $\mathfrak{h}$ coincide com o próprio $\mathfrak{h}$.

Quando $\mathfrak{g}_{\lambda} \neq \emptyset$ diremos que $\lambda$ é uma raiz de $\mathfrak{g}$ e o conjunto de todas as raízes é denotado por $\Phi$. Para todo $\lambda \in \mathfrak{h}^{*}$ (em especial todas raízes) definimos o elemento $t_{\lambda} \in \mathfrak{h}$ como $\lambda(h)=\kappa\left(t_{\lambda}, h\right)$ para todo $h \in \mathfrak{h}$. A existência e unicidade deste elemento decorre do fato da forma de Killing ser não degenerada em $\mathfrak{h}$. Com isto, podemos definir a seguinte forma bilinear em $\mathfrak{h}^{*},(\lambda, \mu)=\kappa\left(t_{\lambda}, t_{\mu}\right)$ para todos $\lambda, \mu \in \mathfrak{h}^{*}$.

A seguinte proposição é um resumo das propriedades de $\Phi$ (g de dimensão finita), ela se encontra em [9], teorema 8.5.

Proposição A.0.8. 1. $\Phi$ gera $\mathfrak{h}^{*}$ como espaço vetorial e $0 \notin \Phi$.

2. Se $\alpha \in \Phi$ então os únicos múltiplos de $\alpha$ em $\Phi$ são $\pm \alpha$

3. Sejam $\alpha, \beta \in \Phi$ então $\beta-2 \kappa(\beta, \alpha) / \kappa(\alpha, \alpha) \alpha \in \Phi$

4.Sejam $\alpha, \beta \in \Phi$ então $2 \kappa(\beta, \alpha) / \kappa(\alpha, \alpha) \in \mathbb{Z}$

Como a expressão $2 \kappa(\beta, \alpha) / \kappa(\alpha, \alpha)$ será muito utilizada, definimos $\langle\beta, \alpha\rangle:=2 \kappa(\beta, \alpha) / \kappa(\alpha, \alpha)$. A propriedade 3 pode ser vista como uma reflexão de $\beta$ em relação ao hiperespaço (subespaço de codimensão 1) normal a $\alpha$ com relação a forma bilinear $\kappa$. Estas propriedades são os axiomas de um sistema de raízes.

Definição A.0.9. Um subconjunto $\Pi$ de $\Phi$ é chamado de base se for linearmente independente e toda raiz $\beta$ pode ser escrita de como $\beta=\sum k_{\alpha} \alpha(\alpha \in \Pi)$ com os coeficientes $k_{\alpha}$ inteiros todos não negativos ou não positivos para cada $\beta$.

O teorema 10.1 de [9] garante que para todo sistema de raízes existe uma base. Definimos a altura de uma raiz $\beta=\sum k_{\alpha} \alpha$ por ht $\beta=\sum k_{\alpha}$, se a altura de uma raiz for positiva (negativa) diremos que a raiz é positiva (negativa, respectivamente). Da definição de base segue que, para uma dada base, o conjunto de raízes se divide em raízes positivas e negativas.

As raízes em $\Pi$ são chamadas raízes simples, fixe uma ordem nestas raízes, digamos $\Pi=$ $\left(\alpha_{1}, \ldots, \alpha_{l}\right)$, onde $l$ é a dimensão de $\mathfrak{h}$. Os elementos $\lambda_{i} \in \mathfrak{h}^{*}$ definidos como $\left\langle\lambda_{i}, \alpha_{j}\right\rangle=\delta_{i j}$ 
são chamados de pesos fundamentais com relação a $\Pi$. A matriz $\left(\left\langle\alpha_{i}, \alpha_{j}\right\rangle\right)_{i j}$, a menos de permutações dos índices, é um invariante de g e é chamada de Matriz de Cartan de $\mathfrak{g}$.

Definição A.0.10. Seja g uma álgebra de Lie. Uma subálgebra de Cartan de g é uma subálgebra $\mathfrak{h} \subset \mathfrak{g}$ que satisfaz

1. hy é nilpotente e

2. o normalizador de $\mathfrak{h}$ em $\mathfrak{g}$ coincide com $\mathfrak{h}$, isto é, $[X, \mathfrak{h}] \subset \mathfrak{h}$ implica que $X \in \mathfrak{h}$.

É importante ubservar que a definição de subálgebra de Cartan não garante sua existência, mas é provada sua existência para álgebras de dimensão finita sobre um corpo infinito. Para álgebras semi-simples, as subálgebras torais maximais serão subálgebras de Cartan.

Exemplo 10. Considere a álgebra $\mathfrak{s l}_{2}(\mathbb{C})$, é um fácil exercício mostrar que está álgebra é simples e não é nilpotente. Mostraremos duas subálgebras de Cartan para esta álgebra. Com a base dada no Exemplo 2 temos que $\mathfrak{h}=\mathbb{C} h$ é uma subálgebra de Cartan. Com efeito, temos que $\mathfrak{h}$ é abeliana, e por consequência é nilpotente. E mais, sejam $a, b, c \in \mathbb{C}$,

$$
[h, a e+b h+c f]=2 a e-2 c f,
$$

que só pertence a h́ se $a=c=0$.

Uma outra álgebra de Cartan de $\mathfrak{s l}_{2}(\mathbb{C})$ é $\mathfrak{h}=\mathbb{C}(e-f)$, novamente $\mathfrak{h}$ é abeliana e

$$
[e-f, a e+b h+c f]=-2 b e-2 b f+(c+a) h,
$$

quie só pertence a í se $b=0$ e $a=-c$.

Exemplo 11. Considere a álgebra $\mathfrak{s l}_{3}(\mathbb{C})$ com a seguinte base

$$
\begin{gathered}
h_{1}=\left(\begin{array}{ccc}
1 & 0 & 0 \\
0 & -1 & 0 \\
0 & 0 & 0
\end{array}\right), \quad h_{2}=\left(\begin{array}{ccc}
0 & 0 & 0 \\
0 & 1 & 0 \\
0 & 0 & -1
\end{array}\right), \quad e_{1}=\left(\begin{array}{lll}
0 & 1 & 0 \\
0 & 0 & 0 \\
0 & 0 & 0
\end{array}\right), \quad e_{2}=\left(\begin{array}{ccc}
0 & 0 & 0 \\
0 & 0 & 1 \\
0 & 0 & 0
\end{array}\right) \\
f_{1}=\left(\begin{array}{lll}
0 & 0 & 0 \\
1 & 0 & 0 \\
0 & 0 & 0
\end{array}\right), \quad f_{2}=\left(\begin{array}{lll}
0 & 0 & 0 \\
0 & 0 & 0 \\
0 & 1 & 0
\end{array}\right), \quad\left[e_{1}, e_{2}\right]=\left(\begin{array}{lll}
0 & 0 & 1 \\
0 & 0 & 0 \\
0 & 0 & 0
\end{array}\right), \quad\left[f_{1}, f_{2}\right]=\left(\begin{array}{ccc}
0 & 0 & 0 \\
0 & 0 & 0 \\
-1 & 0 & 0
\end{array}\right) .
\end{gathered}
$$

O colchete é dado pelo comutador

$$
\left[h_{1}, h_{2}\right]=0,\left[h_{i}, e_{i}\right]=2 e_{i},\left[h_{i}, f_{i}\right]=-2 f_{i},\left[e_{i}, f_{i}\right]=h_{i},\left[h_{i}, e_{j}\right]=-e_{j},\left[h_{i}, f_{j}\right]=f_{j},\left[e_{i}, f_{j}\right]=0,
$$

onde $i \neq j \in\{0,1\}$. As demais relações seguem da identidade de Jacobi.

Com relação a esta base temos

$$
\operatorname{ad}\left(h_{1}\right)=\operatorname{diag}(0,0,2,-1,-2,1,1,-1), \operatorname{ad}\left(h_{2}\right)=\operatorname{diag}(0,0,-1,2,1,-2,1,-1),
$$

onde diag representa a matriz diagonal com as respectivas entradas. É fácil ver que $\kappa\left(h_{i}, h_{i}\right)=12$ e $\kappa\left(h_{1}, h_{2}\right)=-6$.

Temos então, as seguintes raízes:

$$
\alpha_{1}=(2,-1), \alpha_{2}=(-1,2), \alpha_{3}=(-2,1), \alpha_{4}=(1,-2), \alpha_{5}=(1,1), \alpha_{6}=(-1,-1) .
$$

Resolvendo o sistema $\kappa\left(t_{\alpha_{i}}, h_{j}\right)=\alpha_{i}\left(h_{j}\right)$ para $i \in\{1, \ldots, 6\}$ e $j \in 1,2$ obtemos

$$
t_{\alpha_{1}}=\frac{1}{6} h_{1}, t_{\alpha_{2}}=\frac{1}{6} h_{2}, t_{\alpha_{3}}=-\frac{1}{6} h_{1}, t_{\alpha_{4}}=-\frac{1}{6} h_{2}, t_{\alpha_{5}}=\frac{1}{6} h_{1}+\frac{1}{6} h_{2}, t_{\alpha_{6}}=-\frac{1}{6} h_{1}-\frac{1}{6} h_{2} .
$$

Temos $\kappa\left(t_{\alpha_{i}}, t_{\alpha_{i}}\right)=1 / 3$, ou seja todas as raízes têm a mesma norma. E $\kappa\left(t_{\alpha_{1}}, t_{\alpha_{5}}\right)=\kappa\left(t_{\alpha_{2}}, t_{\alpha_{5}}\right)=$ $-\kappa\left(t_{\alpha_{1}}, t_{\alpha_{2}}\right)=1 / 6$. 
Uma possível base para $\Phi$ é $\Pi=\left(\alpha_{1}, \alpha_{2}\right)$. Para esta escolhe de base temos os seguintes pesos fundamentais $\lambda_{1}=(1 / 3)\left(2 \alpha_{1}+\alpha_{2}\right)$ e $\lambda_{2}=(1 / 3)\left(\alpha_{1}+2 \alpha_{2}\right)$. E a seguinte Matriz de Cartan

$$
\left(\begin{array}{cc}
2 & -1 \\
-1 & 2
\end{array}\right)
$$

Uma maneira de representar as raízes e os pesos fundamentais é o seguinte diagrama

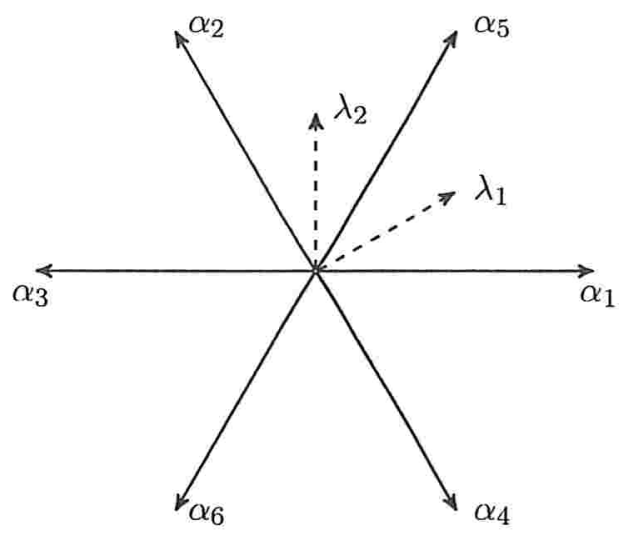

Dada uma matriz de Cartan $A$ de tamanho $k \times k$ podemos recuperar a álgebra de Lie associada a ela via as relações de Chevalley-Serre. Esta álgebra é gerada por $3 k$ geradores $\left\{e_{i}, f_{i}, h_{i}\right\}$ com $i \in\{1, \ldots, k\}$ e as seguintes relações

$$
\begin{gathered}
{\left[h_{i}, h_{j}\right]=0, \quad\left[e_{i}, f_{j}\right]=\delta_{i j} h_{i}, \quad\left[h_{i}, e_{j}\right]=A_{i j} e_{j},} \\
{\left[h_{i}, f_{j}\right]=-A_{i j} f_{j}, \quad\left(\operatorname{ad}\left(e_{i}\right)\right)^{1-A_{i j}}\left(e_{j}\right)=0, \quad\left(\operatorname{ad}\left(f_{i}\right)\right)^{1-A_{i j}}\left(f_{j}\right)=0,}
\end{gathered}
$$

temos ainda que os elementos $\left\{h_{i}\right\}$ geram uma subálgebra de Cartan, para uma prova disso e do que se segue veja [18] páginas 48-55.

Para que a matriz de Cartan seja associada a uma álgebra de Lie simples de dimensão finita temos as seguintes condições necessárias e suficientes:

- As entradas na diagonal de $A$ são iguais a 2,

- As entrada fora da diagonal são não positivas e $A_{i j}=0$ se, e somente se, $A_{j i}=0$,

- A é indecomponível,

- $A$ é simetrizável e a parte simétrica é positiva-definida.

Uma matriz é dita simetrizável quando pode ser escrita como produto de uma matriz diagonal e uma simétrica, esta é chamada de parte simétrica. Matrizes desse tipo são chamadas de matrizes de Cartan do tipo finito.

Se na quarta condição não exigirmos que $A$ seja positiva definida, obtemos uma classe muito maior, as álgebras desta classe são chamadas de álgebras de Kac-Moody.

Uma importante subclasse de álgebras de Kac-Moody são as álgebras do tipo afim. Elas são geradas por matrizes de Cartan do tipo afim que são matrizes que respeitam as quatro condições anteriores (exceto a parte simétrica ser positiva definida) e mais a seguinte condição:

- $\operatorname{det}(A)=0$ e a eliminação de uma linha e a respectiva coluna resulta numa matriz do tipo finito.

Exemplo 12. Álgebras do tipo afim tem dimensão infinita, neste exemplo mostraremos o caso mais simples $\widetilde{\mathfrak{s l}}_{2}$, cuja matriz de Cartan é dada por:

$$
\left(\begin{array}{cc}
2 & -2 \\
-2 & 2
\end{array}\right)
$$


Considere as duas raízes simples $\alpha_{1}$ e $\alpha_{2}$ associadas as trincas $\left\{h_{1}, e_{1}, f_{1}\right\}$ e $\left\{h_{2}, e_{2}, f_{2}\right\}$, das relações de Chevalley-Serre temos

$$
\left[e_{1}\left[e_{1}\left[e_{1}, e_{2}\right]\right]\right]=0 \quad \text { e } \quad\left[e_{2}\left[e_{2}\left[e_{2}, e_{1}\right]\right]\right]=0 .
$$

Obtemos assim as seguintes raízes:

- $e_{3}=\left[e_{1}, e_{2}\right] \neq 0 \Rightarrow \alpha_{1}+\alpha_{2}$ é raíz,

- $e_{4}=\left[e_{1}\left[e_{1}, e_{2}\right]\right] \neq 0 \Rightarrow 2 \alpha_{1}+\alpha_{2}$ é raíz,

- $e_{5}=\left[e_{2}\left[e_{1}, e_{2}\right]\right] \neq 0 \Rightarrow \alpha_{1}+2 \alpha_{2}$ é raíz,

- $\left[e_{1}, e_{4}\right]=\left[e_{1},\left[e_{1},\left[e_{1}, e_{2}\right]\right]\right]=0 \Rightarrow 3 \alpha_{1}+\alpha_{2}$ não é raíz,

- $\left[e_{2}, e_{5}\right]=-\left[e_{2},\left[e_{2},\left[e_{2}, e_{1}\right]\right]\right]=0 \Rightarrow \alpha_{1}+3 \alpha_{2}$ não é raíz,

- $e_{6}=\left[e_{2}, e_{4}\right] \neq 0 \Rightarrow 2 \alpha_{1}+2 \alpha_{2}$ é raíz,

- $e_{7}=\left[e_{2}, e_{6}\right] \neq 0 \Rightarrow 2 \alpha_{1}+3 \alpha_{2}$ é raíz,

Continuando este processo obtemos o seguinte conjunto de raízes

$$
\Phi=\left\{n \alpha_{1}+m \alpha_{2}|n, m \in \mathbb{Z},| n-m \mid \leq 1\right\} .
$$

Observe que diferente do caso finito, múltiplos de raízes podem ser raízes também. 


\section{Apêndice B}

\section{Representações}

De modo superficial, a teoria das representações é o estudo de estruturas algébricas abstratas representando seus elementos como transformações lineares entre espaços vetoriais. Um pouco mais formal, uma representação de uma estrutura algébrica $A$ é um espaço vetorial $V$ com um morfismo $\rho: A \rightarrow \operatorname{End}(V)$, isto é, uma aplicação que preserva a estrutura algébrica.

A teoria das representações surgiu em 1896 em um trabalho de F. G. Frobenius. para resolver um problema enviado por R. Dedekind. Este problema e a resolução apresentada quanto outros dados da história da teoria das representações podem ser encontradas em [ڤ̄].

O interesse desta teoria é tornar objetos abstratos mais concretos, tornando problemas de álgebra abstrata, muitas vezes, em problemas de álgebra linear.

No exemplo 6 vimos que uma álgebra associativa pode ser transformada em uma álgebra de Lie. Como $E n d(V)$ é uma álgebra associativa, a imagem da álgebra de Lie via uma representação é uma subálgebra de Lie não necessariamente associativa. Porém. a álgebra associativa gerada por ela contêm muitas informação sobre a representação. Gostaríamos de construir uma estrutura que carregasse esta informação e para toda representação da álgebra de Lie tivéssemos uma representação pra esta estrutura.

Esta estrutura é chamada de álgebra universal envolvente. Vamos mostrar sua construção. Seja $\mathfrak{g}$ uma álgebra de Lie sobre um corpo $k$. Considere os seguintes conjuntos

$$
T^{0} \mathfrak{g}=k, \quad T^{i} \mathfrak{g}=\mathfrak{g} \otimes T^{i-1} \mathfrak{g},
$$

isto é $T^{0} \mathfrak{g}$ é o corpo e $T^{k} \mathfrak{g}$ consiste de elementos que estejam no produto tensorial de $i$ cópias de $\mathfrak{g}$. Considere o espaço

$$
T(\mathfrak{g})=\bigoplus_{i=0}^{\infty} T^{i} \mathfrak{g}
$$

com a multiplicação de monômios dada pelo isomorfismo canônico:

$$
T^{i} \mathfrak{g} \otimes T^{j} \mathfrak{g} \simeq T^{i+j} \mathfrak{g} .
$$

Considere o ideal $I \subset T(\mathfrak{g})$ gerado por todos elementos da forma

$$
a \otimes b-b \otimes a-[a, b],
$$

onde $a, b \in \mathfrak{g}$. A álgebra universal envolvente, $U(\mathfrak{g})$ é dada pelo quociente

$$
U(\mathfrak{g}):=T(\mathfrak{g}) / I .
$$

Observe que $\iota: U(\mathfrak{g}) \rightarrow \mathfrak{g}$ definido como

$$
\iota(a)=a,
$$


onde $a \in \mathfrak{g}$ é um epimorfismo. Além disso, a imagem de $\mathfrak{g}$ por $\iota$ gera $U(\mathfrak{g})$ como álgebra.

O termo universal decorre da seguinte propriedade universal: Dado um homomorfismo $\rho: \mathfrak{g} \rightarrow A$, onde $A$ é uma álgebra associativa, existe único homomorfismo $\widetilde{\rho}: U(\mathfrak{g}) \rightarrow A$ tal que $\rho=\tilde{\rho}$. Este último homomorfismo no sentido de preservar a estrutura de álgebra associativa, isto é, $X, Y \in U(\mathfrak{g})$,

$$
\widetilde{\rho}(X Y)=\widetilde{\rho}(X) \widetilde{\rho}(Y) .
$$

O seguinte teorema conhecido como teorema PBW fornece uma descrição precisa da estrutura de $U(\mathfrak{g})$. Em especial, podemos ver (uma cópia de) $\mathfrak{g}$ como um subespaço de $U(\mathfrak{g})$.

Teorema B.0.11 (Poincaré-Birkhoff-Witt). Seja g uma álgebra de Lie (de dimensão finita ou não) $e\left\{X_{i}\right\}_{i \in J}$ uma base de g ordenada por uma ordem no conjunto dos indices $J$. Então, os monômios do tipo

$$
X_{i_{1}} \ldots X_{i_{k}}, \quad i_{1} \leq \ldots \leq i_{k}
$$

formam uma base de $U(\mathfrak{g})$. 


\section{Referências Bibliográficas}

[1] S. Berman and Y. Billig. Irreducible representations for toroidal lie algebras. J. Algebra, 221 (1):188-231, 1999. 1, 21

[2] Y. Billig. A category of modules for the full toroidal lie algebra. International Mathematics Research Notices, (Art. ID 68395):46pp, 2006. 1, 9, 19, 21, 22

[3] Y. Billig and V. Futorny. Representations of the lie algbra of vector fields on a torus and chiral de rham complex. Transactions of the American Mathematical Society, 130(1):77-117, 2013. $1,11,23,24,26,27$

[4] Y. Billig, A. Molev, and R. Zhang. Differential equations in vertex algebras and simple modules for the lie algebraof vector fields on a torus. Adv. Math, 218:1972-2004, 2008. 1, 23

[5] C. W. Curtis. Pioneers of Representation Theory. American Mathematical Society and London Mathematical Society, 2010. 37

[6] C. Dong, H. Li, and G. Mason. Vertex lie algebras, vertex poisson algebras and vertex algebras. Contemp. Math., 297:69-96, 2002. 17

[7] X. Guo and K. Zhao. Irreducible weight modules over witt algebras. American Mathematical Society, 139(7):2367-2373, 2011. 12

[8] Q. He. Lie algebras and the lie brackets of lie groups-matrix groups. http://www.math. uchicago.edu/ may/VIGRE/VIGRE2011/REUPapers/He.pdf. 32

[9] J. E. Humphreys. Introduction to Lie Algebra and Representation Theory. Springer, 1972. 3, 31,33

[10] V. Kac. Infinite Dimensional Lie Algebras. Cambridge University Press, 1994. 31

[11] V. Kac. Vertex Algebra for Begginers. American Mathematical Society, 1998. 15

[12] V. G. Kac and D. A. Kazhdan. Structure os representations with hightest weight of infintedimensional lie algebras. Advances in Mathematics, 34:97-108, 1979. 5

[13] F. ten Kroode and J. van de Leur. Bosonic and fermionic realization of the affine algebra $\widehat{\mathfrak{g l}}$. Comm. Math. Phys., 137:67-107, 1991. 28

[14] H. Li. Local systems of vertex operators, vertex superalgebras and modules. J. Pure Appl. Algebra, 109:143-195, 1996. 15

[15] F. Malikov, V. Schechtman, and A. Vainntrob. Chiral de rham complex. Comm. Math Phys, 204:439-473, 1999. 27

[16] S. E. Rao. Irreducible representations of the lie algebra of the diffeomorphisms of a ddimensional torus. J. Algebra, 182(1):401-421, 1996. 12

[17] L. A. B. San Martin. Álgebras de Lie. Editora Unicamp, 2010. 31

[18] J. P. Serre. Complex Semisimple Lie Algebras. Springer, 1987. 35 
DOAÇÃO

De: $\mathrm{CPG}$ - IMEUSPP

Data Rec.: 17.07.2015

Orient:. Profor. Vyachesslav Futhriny

Tamban $20-0 \%-15$

BIBLIOTECA "CARLOS BENIAHAIN LYRA"

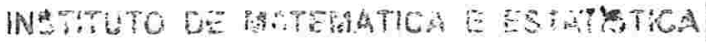

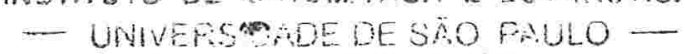

CIDADE UTVERSTAR:A

C P 65.281-A Cidede co Sap patho

$05314-970$ - SAO PALLO ERASIL

Te $3084-6174,3091-0109.3091-6283$

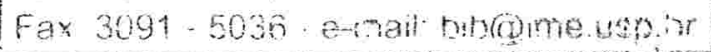

